\title{
Efficient Photoacoustic Conversion in Optical Nanomaterials and Composites
}

\author{
Taehwa Lee, Hyoung Won Baac, Qiaochu Li, and L. Jay Guo*
}

Photoacoustic pulses generated by pulsed laser irradiation have the characteristics of high frequency and wide bandwidth, which are desirable for imaging and sensing. Efficient photoacoustic composites have been developed for fabricating photoacoustic transmitters capable of generating highamplitude ultrasound. Here, recent advances in photoacoustic transmitters are reviewed from an application perspective, starting with the fundamental aspects of photoacoustic generation. The topics discussed include various composite materials for photoacoustic generation, and their applications such as high-amplitude therapy, imaging and sensing, and photoacoustic waveform control.

\section{Introduction}

Photoacoustic conversion using the photoacoustic (or optoacoustic) effect allows for generation of high-frequency and broadband ultrasound. Previously such high-frequency photoacoustic signals have been exclusively implemented for functional imaging applications requiring high spatial resolution, since the imaging resolution depends on ultrasound frequency. There exist a myriad of imaging applications including material defect characterization $^{[1]}$ and photoacoustic imaging of biological tissues. ${ }^{[2]}$

Beyond photoacoustic imaging, emerging applications of the photoacoustic effect have been recently proposed owing to newly developed photoacoustic materials showing efficient light to sound energy conversion. Pulsed optical excitation of these efficient photoacoustic materials results in ultrasound pulses with high amplitudes as well as high frequency. In contrast, ultrasound pulses having both high amplitudes and high frequency are difficult to attain with traditional piezoelectric-based

Dr. T. Lee, Prof. L. J. Guo

Department of Mechanical Engineering

University of Michigan

Ann Arbor, MI 48109, USA

E-mail:guo@umich.edu

Prof. H.W. Baac

Department of Electrical and Computer Engineering

Sungkyunkwan University

Suwon 16419, Republic of Korea

Q. Li, Prof. L. J. Guo

Department of Electrical Engineering and Computer Science

University of Michigan

Ann Arbor, MI 48109, USA

The ORCID identification number(s) for the author(s) of this article can be found under https://doi.org/10.1002/adom.201800491.

DOI: 10.1002/adom.201800491 approaches. While addressing the limitations of the electrical counterparts (e.g., massive amounts of radio frequency $(\mathrm{RF})$ cabling and electromagnetic (EM) interference), a photoacoustic approach for generating high-amplitude ultrasound has created new opportunities.

Active research on efficient photoacoustic materials has started with the development of photoacoustic contrast mediums (e.g., exogenous contrast particles) for contrast enhancement. ${ }^{[3-5]}$ Recent advances in nanofabrication technologies have allowed to develop more efficient photoacoustic contrast mediums, ${ }^{[3]}$ such as metal nanoparticles having enhanced optical absorption due to optical resonance. Later, by coating such nanoparticles on a flat substrate, layered structures were developed for generating high-amplitude ultrasound. Among many nanoparticles, gold nanoparticles were widely used owing to strong resonance. ${ }^{[6]}$ In fact, these initial efforts to increase photoacoustic conversion were focused on increasing light absorption, while the critical role of thermal expansion for photoacoustic generation were often overlooked.

To enhance thermal expansion for photoacoustic generation, researchers have started to fabricate composite materials, composed of light-absorbing materials mixed with polymers with high thermal expansion coefficients. These polymer-based composites significantly enhance photoacoustic conversion efficiency. Among many polymers, polydimethylsiloxane (PDMS) is exclusively used because of its high thermal expansion, optical transparency, and acoustic impedance comparable to that of water. ${ }^{[7-11]}$ Photoacoustic pulses generated with PDMSbased composites have the characteristics of high amplitude and high frequency, which have enabled interesting applications such as cavitation-based therapy ${ }^{[12]}$ and imaging. ${ }^{[13]}$ For photoacoustic composites, there are a myriad of light-absorbing nanomaterials such as metal absorbers ${ }^{[6]}$ and carbon nanomaterials (e.g., carbon black $(\mathrm{CB}),{ }^{[14]}$ carbon nanotube $(\mathrm{CNT}),{ }^{[7,10]}$ carbon nanofiber $(\mathrm{CNF}),{ }^{[9]}$ candle soot $(\mathrm{CS}),{ }^{[8]}$ and reduced graphene oxide $\left.(\mathrm{rGO})^{[15]}\right)$. These light-absorbing materials of nanometer sizes have an advantage over microscale absorbing materials ${ }^{[7]}$ in facilitating heat transfer from the light absorbers into the surrounding mediums. Such facilitated heat transfer is another key factor that contributes to photoacoustic energy conversion.

In this Review, we will start with general considerations of photoacoustic generation in nanomaterial composites. Various photoacoustic composites enabling interesting applications will be introduced. In addition, recent efforts will be reviewed from an application perspective. Also, we will discuss outlook and future directions. 


\section{Photoacoustic Generation-Mechanism and General Considerations}

\subsection{Photoacoustic Generation Mechanism}

Light-to-sound conversion can occur either by light-induced material ablation or thermoelastic effect, as illustrated in Figure 1. For high intensity light pulses, the temperature of a light-absorbing material can exceed its melting or boiling temperature, and thus the material experiences phase change. The resulting material ablation exerts recoil momentum to the material, generating photoacoustic waves. Such ablationinduced photoacoustic waves have relatively high photoacoustic amplitudes, but the ablation process is destructive to the constitute materials (Figure 1d). In contrast, photoacoustic generation via the thermoelastic effect is a nondestructive approach, which does not cause any light-induced damages to materials. For relatively low intensity light pulses, photoacoustic generation relies on the thermoelastic effect. Photoacoustic materials convert light into heat, leading to transient thermal expansion in the materials and acoustic generation (Figure 1c). One important requirement for photoacoustic generation is that optical heating of a material should be faster than the thermal expansion of the heated material (the so-called stress confinement condition). To meet this requirement, incident optical energy should be applied to a photoacoustic material in the form of short pulses or intensitymodulated waves.

The speed of thermoelastic volume expansion can be correlated to photoacoustic pressure amplitude through momentum conservation, ${ }^{[16]}$ which can be given by $P_{0} \cong \rho c U$, where $\rho$ is the mass density $\left(\mathrm{kg} \mathrm{m}^{-3}\right), c$ is the sound speed $\left(\mathrm{m} \mathrm{s}^{-1}\right)$, and $U$ is the speed of thermoelastic volume expansion $\left[U=\Delta V / S \tau_{l}\right.$, $\Delta V$ : thermoelastic volume expansion $\left(\mathrm{m}^{3}\right), S$ : surface area $\left(\mathrm{m}^{2}\right)$, and $\tau_{l}$ : laser pulse duration (s)]. By using the thermoelastic volume expansion defined as $\Delta V=A \cdot S \cdot F \cdot \beta / \rho C_{\mathrm{p}}$ with $A$ the light absorption $(0<A<1$; dimensionless), $F$ the laser fluence $\left(\mathrm{J} \mathrm{m}^{-2}\right), \beta$ the volumetric thermal-expansion coefficient $\left(\mathrm{K}^{-1}\right)$, and $C_{\mathrm{p}}$ the specific heat capacity at constant pressure $\left(\mathrm{Jg}^{-1} \mathrm{~K}^{-1}\right)$, the photoacoustic signal amplitude $(\mathrm{Pa})$ can be rewritten as

$P_{0}=\Gamma \cdot A \cdot \frac{F}{l}$

where $\Gamma=\beta c^{2} / C_{\mathrm{p}}$ is the dimensionless Grüneisen parameter, $l$ is the characteristic length $(\mathrm{m})$, which will be discussed in the following section. It is commonly believed that strong light absorption $(A)$ and high thermal expansion $(\beta)$ is needed for high photoacoustic amplitudes. More precisely, Equation (1) indicates that $P_{0}$ is determined by $\Gamma$ (instead of $\beta$ alone) and the energy volume density $(A \cdot F / l)$ in $\mathrm{J} \mathrm{m}^{-3}$ (or $\mathrm{Pa}$ ).

\subsection{Theory of Photoacoustic Generation}

Although Equation (1) provides reasonable order-of-magnitude estimation, the photoacoustic amplitude can be more rigorously calculated by simultaneously solving heat conduction equation and pressure wave equation. ${ }^{[17,18]}$ The heat conduction equation

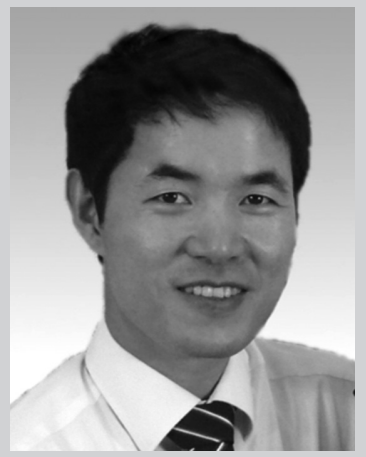

Taehwa Lee received a Ph.D. degree in the Department of Mechanical Engineering from the University of Michigan, Ann Arbor, USA, in 2015. He was a postdoctoral research Fellow 2016-2017 in the Molecular Imaging Program at Stanford University. His research interests lie in energy conversion phenomena with focus on

wave-matter interactions such as light-matter interaction for sound generation, and coupling of acoustic waves with structural vibration. Also, his interests include medical ultrasound, cavitation generation/monitoring, and acoustic metamaterials.

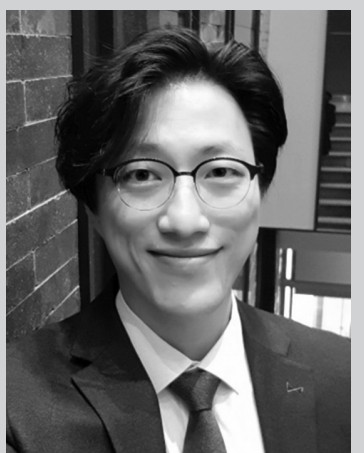

Hyoung Won Baac received a Ph.D. degree in the Department of Electrical Engineering and Computer Science from the University of Michigan, Ann Arbor, USA, in 2012. From 2012 to 2014 , he was a research fellow with Harvard Medical School, Wellman Center for Photomedicine, and Massachusetts General Hospital. Since 2014, he has been an assistant professor with the Department of Electrical and Computer Engineering, Sungkyunkwan University, Republic of Korea. His main research interest is the development of lasergenerated focused ultrasound (LGFU) systems, optoacoustic transmitters from miniature to macroscale, and all-optical ultrasound transducers for precision therapy and diagnostic applications.

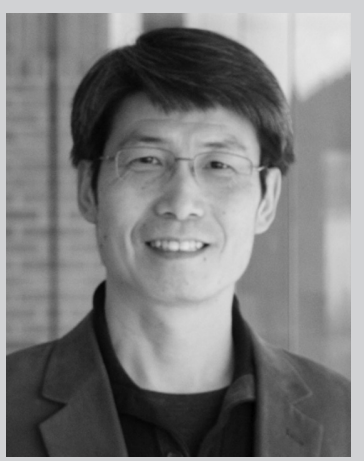

L. Jay Guo started his academic career at the University of Michigan in 1999, where he is currently a professor of electrical engineering and computer science since 2011 , with joint appointment in applied physics, mechanical engineering, macromolecular science and engineering. He directs an interdisciplinary laboratory at the intersection of electrical engineering, photonics, polymer materials, and mechanical engineering. His group's researches include polymer-based photonic devices and sensor applications, organic and hybrid photovoltaics, plasmonic nanophotonics, and scalable nanomanufacturing, such as roll-to-toll nanomanufacturing technologies. 
(a)

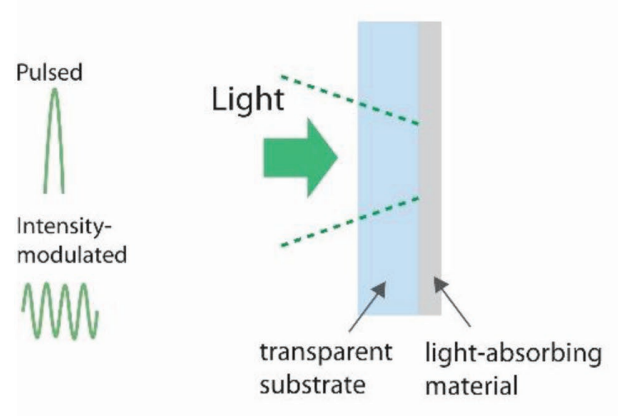

(c)

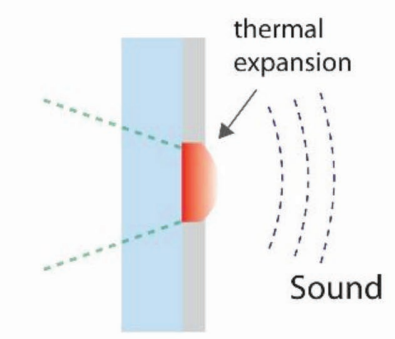

Thermoelastic regime (b)

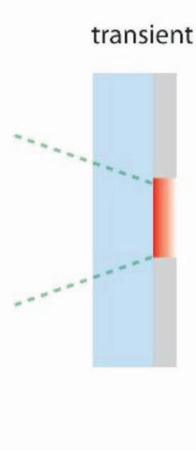

(d)

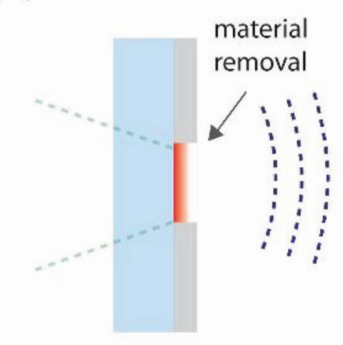

Ablation regime

Figure 1. Photoacoustic generation. a) Light absorption in a light-absorbing material. Light is illuminated in the form of pulsed or intensity-modulated waves. b) Transient heating of the material by light absorption. Sound generation through two different regimes: c) thermoelastic regime and d) ablation regime. In the thermoelastic regime, sound is generated by thermal expansion of the heated material. In the ablation regime, the parts of the material are removed through phase change (invasive), producing much stronger sound intensity.

yields the time-dependent temperature field $T(\vec{r}, t)$ caused by pulsed-laser heating, which is represented by

$\rho C_{\mathrm{p}} \frac{\partial T(\vec{r}, t)}{\partial t}=\nabla(k \nabla T)+H(\vec{r}, t)$

where $k$ is the thermal conductivity $\left(\mathrm{W} \mathrm{m}^{-1} \mathrm{~K}^{-1}\right)$, and $H(\vec{r}, t)$ is the volumetric nonradiative heat generation due to light absorption $\left(\mathrm{W} \mathrm{m}^{-3}\right)$. The above equation is based on the assumption that adiabatic heating induced by compressive pressure is negligible when $C_{\mathrm{p}}$ is almost identical to the specific heat capacity at constant volume $C_{\mathrm{V}} \cdot{ }^{[2]}$ With the calculated temperature from Equation (2), the wave equation can be solved for the pressure field $p(\vec{r}, t)$ in a fluid as

$\left[\nabla^{2}-\frac{1}{c^{2}} \frac{\partial^{2}}{\partial t^{2}}\right] p(\vec{r}, t)=Q_{\text {aco }}$

where $c$ is the sound speed, $Q_{\text {aco }}$ is the acoustic source. Here, the acoustic source is modeled by the thermoelastic mechanism, i.e., $Q_{\text {асо }}=-\rho \beta \frac{\partial^{2} T}{\partial t^{2}}$.

Under the assumption of negligible heat conduction (i.e., $\nabla(k \nabla T)=0$ ), Equation (2) is simplified to $\rho C_{\mathrm{p}} \frac{\partial T(\vec{r}, t)}{\partial t}=H(\vec{r}, t)$. Thus, the acoustic source can be determined where $f(t)$ is the temporal heating function, $g(z)$ is spatial light absorption function, * represents the convolution integral, $\tau$ is the retardation time $(\tau=t-z / c)$, and $z$ is the distance along the $z$ direction). ${ }^{[17]}$ The convolution integral for pressure estimation is valid for negligible heat conduction during optical excitation such that the spatial heat source is dictated by the spatial light absorption function. In this case, Equation (5) is derived from the Green's function solution of Equation (4). $f(t)$ is as a function of the laser pulse duration $\left(\tau_{l}\right)$, while $g(z)$ as a function of the light absorption coefficient $(\alpha)$.

Equation (5) does not only provide good approximation, but also gives some insights into photoacoustic generation. To understand the convolution integral of the two functions in Equation (5), we start by considering two scenarios: thin absorbers and thick absorbers, as illustrated in Figure 2a. Suppose that a light pulse with a gaussian temporal profile $f(t)$ is irradiated onto a thin or thick absorber, each absorbing $100 \%$ of the incident optical energy. For the sake of simplicity, the light absorption profile $g(z)$ of each absorber is spatially uniform (rectangular function), but has different depths $\left(1 / \alpha_{\text {thin }}\right.$ for the thin absorber, and $1 / \alpha_{\text {thick }}$ for the thick absorber), i.e., $g(z)=g_{0}(H(0)-H(z-1 / \alpha))$, with $H(z)$ the Heaviside function $\left(H\left(z-z_{0}\right)=0\right.$ for $z<z_{0}$ or 1 for $\left.z>z_{0}\right)$, $\mathrm{g}_{0}$ is the light absorption amplitude. For light pulses of the same fluence $F$, the light absorption amplitude $g_{0}$ of the thin 
(a)

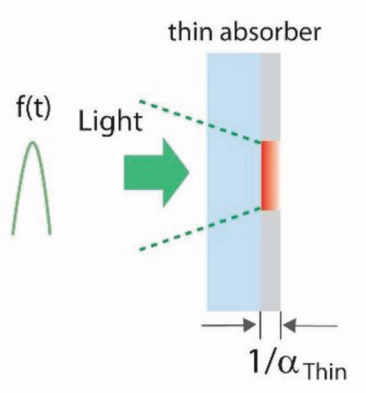

(b)
Convolution integral $(f * g)$

Temporal light

$f(t)$

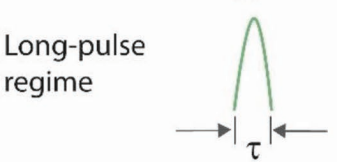

$f(t)$

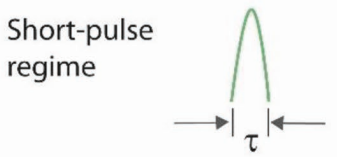

*
Spatial absorption

$g(z)$

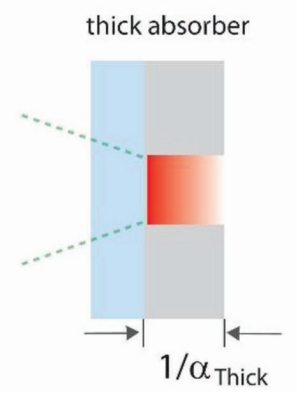

Resulting pressure pulse

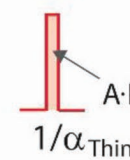

$=$

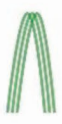

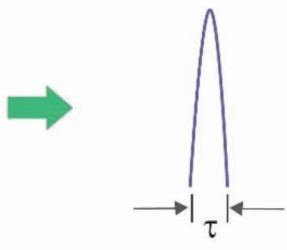

$g(z)$
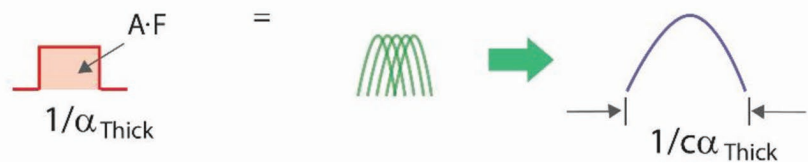

Figure 2. Photoacoustic pulse regimes. a) Photoacoustic generation for thin and thick absorbers. b) Photoacoustic pulses determined with convolution integral for long pulse (top) and short pulse (bottom) regimes.

absorber is much larger than that of the thick absorber, i.e., $F=g_{0, \text { thin }} / \alpha_{\text {thin }}=\mathrm{g}_{0, \text { thick }} / \alpha_{\text {thick }}$. Figure $2 \mathrm{~b}$ illustrates the convolution integral for the two absorbers. Recall the assumption of negligible heat conduction during the optical excitation, then the light absorption depth is identical to the depth of the heat source. The convolution integral means that the heat source is virtually divided into thin slices, each single slice emits a sound wave with the same temporal profile as $f(t)$. Thus, the superposition of the sound waves results in the final photoacoustic wave. For the thin absorber, the heat source emits fewer numbers of sound waves of higher amplitudes $\left(\approx g_{0, \text { thin }}\right)$, whereas the heat source for the thick absorber generates more numbers of sound waves of lower amplitudes $\left(\approx g_{0, \text { thick }}\right)$. For the thin absorber, the resulting photoacoustic waves have high amplitudes and narrow pulse width (nearly identical to that of light pulses $\tau_{l}$, more precisely, $\left.\tau_{l}+1 / c \alpha_{\text {thin }}\right)$, while for the thick absorber, the photoacoustic waves have low amplitudes and wide pulse width $\left(\approx \tau_{l}+1 / c \alpha_{\text {thick }}\right)$. In summary, the width of the resulting photoacoustic wave corresponds to $\tau_{l}+1 / c \alpha$.

From the convolution integral [Equation (5)], the pulse width of the photoacoustic wave is determined with $\tau_{l}+1 / c \alpha$, which corresponds to $l=c \tau_{l}+1 / \alpha$ in length scale. The length $l$ is referred to as the characteristic length. Plugging the characteristic length into Equation (1), the photoacoustic pressure amplitude is given by

$$
P_{0}=\Gamma \cdot A \cdot \frac{F}{c \tau_{l}+1 / \alpha}
$$

The condition $c \tau_{l} \gg 1 / \alpha$ (i.e., $l \sim c \tau_{l}$ ), commonly observed in thin absorbers, is referred to as long pulse regime because the light pulse $\left(\tau_{l}\right)$ is much longer than $1 / c \alpha$. In contrast, the condition $c \tau_{l} \ll 1 / \alpha$ (i.e., $l \sim 1 / \alpha$ ) for thick absorbers is referred to as short pulse regime. In these cases, the photoacoustic pressure amplitude is expressed by

$$
\begin{aligned}
P_{0} & =\Gamma \cdot A \cdot \frac{F}{\max \left(c \tau_{l}, 1 / \alpha\right)} \\
& =\left\{\begin{array}{l}
\left.\Gamma \cdot A \cdot \frac{F}{c \tau_{l}} \text { (long pulse regime; } \quad c \tau_{l} \gg 1 / \alpha\right) \\
\left.\Gamma \cdot A \cdot \frac{F}{1 / \alpha}=\Gamma \cdot A \cdot \alpha F \text { (short pulse regime; } \quad c \tau_{l} \ll 1 / \alpha\right)
\end{array}\right.
\end{aligned}
$$

The formula for the short pulse regime is found elsewhere. ${ }^{[13]}$ The absorption coefficient $(\alpha)$ defines the length scale for the energy volume density rather than light absorption, since $A$ represents light absorption.

The estimation stated above could provide an intuitive way to guide practical applications in many simple scenarios. Comprehensive theoretical studies on photoacoustic generation have been reported in the literature..$^{[17,19-22]}$ And recent theoretical studies can be found elsewhere. ${ }^{[23-27]}$ 


\subsection{Heat Transfer in Nanocomposites}

Owing to their high thermal expansion coefficients, polymers exhibit more efficient photoacoustic conversion than metals when the same light absorption is assumed. However, these polymers are optically transparent, requiring light-absorbing fillers. ${ }^{[7,8]}$ In such case, heat transfer from the absorbers to the surrounding polymer is critical to photoacoustic conversion.

To understand the heat transfer in these nanocomposites, one can consider a film consisting of a thin light-absorbing layer sandwiched by two transparent polymer layers. The thin middle layer absorbs the incident light, which is subsequently converted into heat, some of which is transmitted into the adjacent polymer layers. According to energy conservation, the fraction of the thermal energy $(\gamma=$ thermal energy in each layer/absorbed optical energy) is represented by

$\frac{F_{\mathrm{A}}}{A \cdot F}+\frac{F_{\mathrm{P}}}{A \cdot F}=\gamma_{\mathrm{A}}+\gamma_{\mathrm{P}}=1$

where the subscript $A$ and $P$ represent the absorbing layer and polymer layer, respectively. Output photoacoustic amplitude is determined by combining photoacoustic amplitudes produced in each layer. Using Equation (1) and $\gamma$, the output photoacoustic amplitude can be expressed by

$P_{0}=P_{\mathrm{A}}+P_{\mathrm{P}}=A \cdot F\left[\gamma_{\mathrm{A}}\left(\frac{\Gamma}{l}\right)_{\mathrm{A}}+\gamma_{\mathrm{P}}\left(\frac{\Gamma}{l}\right)_{\mathrm{P}}\right]$

If the contribution of the absorber is negligible $\left(P_{\mathrm{A}} / P_{\mathrm{P}} \ll 1\right)$, the resulting photoacoustic amplitude is given by the photoacoustic amplitude in the polymer

$P_{0} \cong P_{\mathrm{P}}=A \cdot F \cdot \gamma_{\mathrm{P}}\left(\frac{\Gamma}{l}\right)_{\mathrm{P}}$

The negligible contribution of the absorber is true when the absorber is made of materials with low thermal expansion coefficients, e.g., metals or carbon-based materials. In this case, the thermal energy in the absorber $\left(A \cdot F \cdot \gamma_{\mathrm{A}}\right)$ is just wasted. To maximize the photoacoustic amplitude, heat transfer should be facilitated for reducing $\gamma_{A}$ and thereby increasing $\gamma_{P}$.

The thermal energy fractions can be calculated by solving the heat conduction equation. Alternatively, the fraction of the thermal energy in the polymer can be estimated as ${ }^{[16]}$

$$
\gamma_{\mathrm{P}}=\frac{1}{\frac{\left(\rho C_{\mathrm{p}}\right)_{\mathrm{A}} d_{\mathrm{A}}}{\left(\rho C_{\mathrm{p}}\right)_{\mathrm{P}} l_{\mathrm{th}, \mathrm{P}}}+1}
$$

where $l_{\text {th,P }}$ is the heat penetration depth of the polymer $l_{\mathrm{th}, \mathrm{P}}=$ $\left(1.269 \alpha_{\mathrm{th}} \tau_{l}\right)^{1 / 2}$ and $d_{\mathrm{A}}$ is the thickness of the absorber layer. Equation (11) states that the ratio of the heat capacities is critical. Thus, $\gamma_{\mathrm{P}}$ can be increased by decreasing the heat capacity of the absorber (by decreasing $d_{\mathrm{A}}$ ). Also, notably, the heat capacity of the polymer layer is proportional to $\tau_{l}$ and thus $l_{\mathrm{th}, \mathrm{P}}$, indicating that larger $\tau_{l}$ is better for increasing $\gamma_{\mathrm{p}}$. From a heat transfer perspective, nanoscale absorbers (small $d_{\mathrm{A}}$ ) are considered an excellent choice because of low heat capacity.

\subsection{General Considerations}

Photoacoustic waves generated by pulsed optical excitation are characterized as short, broadband pulses. In addition to these characteristics, high amplitude can be obtained by proper material selection and structure design. Depending on target applications, desired characteristics of photoacoustic pulses may be varied. However, usually, high-amplitude broadband pulses are desirable. For example, high frequency is important for imaging applications, because it defines imaging resolution, as well as attenuation (or imaging depth). Moreover, high amplitude is required for therapeutic applications using ultrasound-induced nonlinear effects such as shock wave and cavitation. Such high amplitude is also desired to increase signal-to-noise ratio for high-resolution imaging.

\section{1) Bandwidth}

Laser Pulse Duration: To achieve desired photoacoustic pulses, one should first choose a laser system capable of delivering the right pulse width, which determines the upper limit of achievable frequency band. Typically, nanosecond lasers produce photoacoustic pulses of tens of $\mathrm{MHz}$ frequency.

Thickness of Composites: According to Equation (5), the bandwidth of photoacoustic pulses is not only determined by laser pulse width, but also by the spatial absorption profile. In the long pulse regime [Equation (7)], where a very thin layer is used, one can reach the upper limit set by the laser pulse width. When a light-absorbing layer has a large thickness, the bandwidth of photoacoustic pulses is smaller than the upper limit.

\section{2) High Amplitude}

High Incident Laser Fluence: Increasing the energy of the incident laser beam is an easy approach to increasing photoacoustic amplitudes. However, this approach is limited by potential material damages induced by laser ablation. Thus, such approach is effective only when light-absorbing materials are sufficiently robust.

Conversion Efficiency: The light-to-sound conversion efficiency of photoacoustic materials needs to be increased for high-amplitude photoacoustic pulses. When the incident laser fluence is limited because of material damage or laser system itself, the conversion efficiency needs to be increased for generating high-amplitude photoacoustic pulses.

Focusing with Large Bandwidth: To produce high-amplitude photoacoustic pulses, one can implement a focusing scheme, where photoacoustic pulses are concentrated to a (focal) spot. The photoacoustic amplitudes at the focal spot are significantly increased, which are characterized with a focal gain multiplied by photoacoustic amplitude right on the source. The higher focal gain is, the higher photoacoustic amplitude is. The focal gain is determined with the geometry of the photoacoustic transmitter (i.e., $f$-number). Also, the focal gain is related to frequency bandwidth such that wide bandwidths (or short pulses) are desired for high focal gains. 
(a)

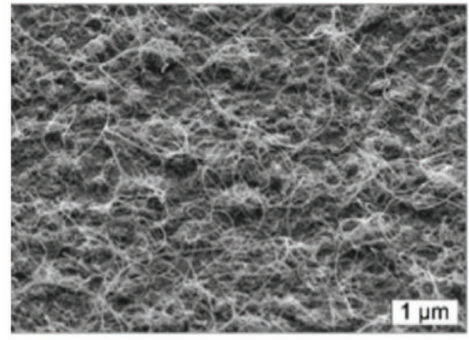

side view

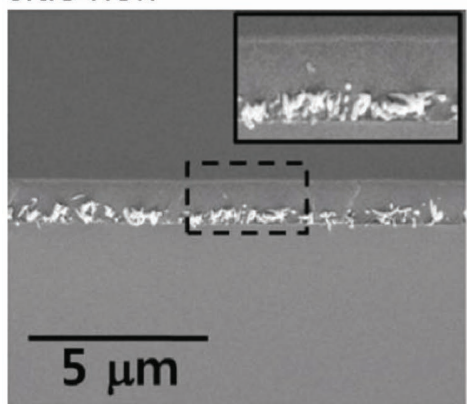

(b)

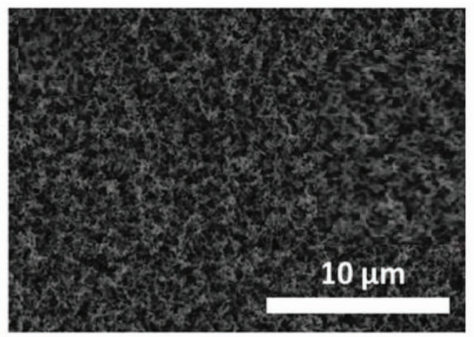

side view

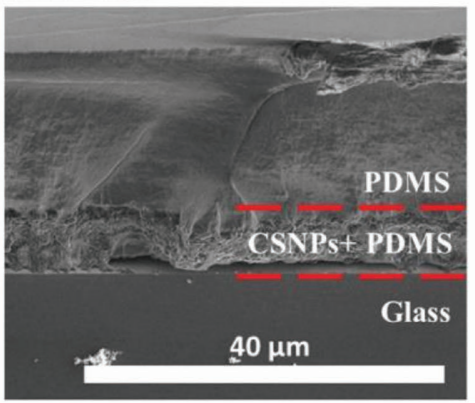

(c)

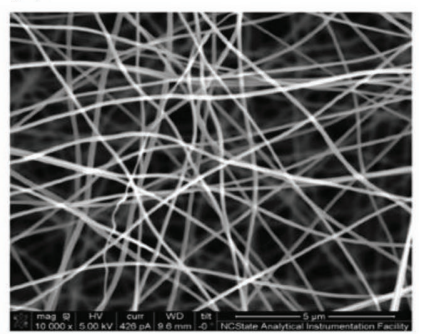

(d)

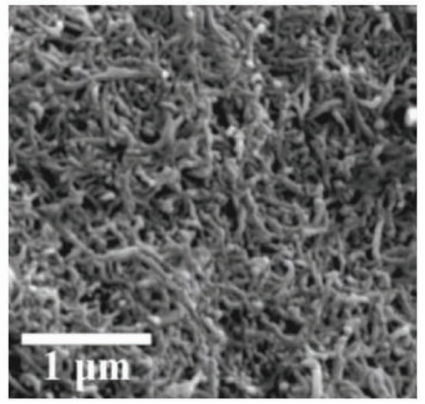

Figure 3. Carbon-based photoacoustic materials. a) CNT-PDMS composites (top: top view image; bottom: side view image). Adapted with permission. ${ }^{\text {[29] }}$ Copyright 2015, The Royal Society of Chemistry. Adapted with permission. [7] Copyright 2010, AIP Publishing LLC. b) Carbon soot nanoparticles (CSNPs)PDMS composites. Adapted with permission. ${ }^{[8]}$ Copyright 2015, AIP Publishing LLC. c) Carbon nanofibers-PDMS composites. Adapted with permission. ${ }^{[9]}$ Copyright 2015, AIP Publishing LLC. d) Solution-processed CNT-PDMS composite. Adapted with permission. ${ }^{11]}$ Copyright 2016 , The Authors.

\section{Highly Efficient Photoacoustic Conversion Materials}

High light-to-sound conversion in photoacoustic materials requires high light absorption and high heat-to-sound conversion efficiency. Originally, research had focused on materials having high light absorption. Later, researchers identified that high thermal expansion is critical to heat-to-sound conversion. Since light-absorbing materials typically do not have high thermal expansion coefficients, researchers started to mix these materials with polymer materials with high thermal expansion coefficients. Recent advances in material science provide allow numerous light-absorbing materials, which include carbon-based materials, and metal-based materials, as shown in Figures 3 and 5. These materials have their own merits for photoacoustic conversion. In terms of optical performance, the carbon-based materials appear black, indicating broadband optical absorption, which allows flexibility in choosing a laser system for optical excitation. In contrast, the metal-based materials have a resonance characteristic, showing high optical absorption at a resonance frequency while having a low optical absorption in an off-resonance condition. Such frequency-dependent absorption can benefit certain applications that require photoacoustic materials with selective optical transmission.

\subsection{Carbon-Based Materials for Broadband Absorption}

\subsubsection{Carbon Nanotube (As Grown)}

Since the first nanostructured transmitter was proposed by using 2D arrays of patterned gold nanoparticles, ${ }^{[6]}$ a substantial progress in generation of high-amplitude high-frequency ultrasound has been made by employing a carbon-nanotube (CNT)PDMS composite as a photoacoustic transmitter. ${ }^{[7]} \mathrm{A}$ thin layer of densely packed CNTs serves as a perfect broadband optical absorber because it provides an excellent impedance matching with air by showing that the top surface of grown CNTs is irregular with subwavelength roughness. Also, such roughness leads to diffused reflection, thus trapping the light. ${ }^{[28]}$

We demonstrated a CNT-PDMS transmitter, where multiwalled (MW) CNTs are well embedded in the PDMS. ${ }^{[7,29]}$ Here, MW CNTs were grown on a transparent substrate using high-temperature chemical vapor deposition process (a hightemperature durable substrate for CNT growth is needed). The as-grown CNT film on a fused silica substrate was then combined with PDMS by spin-coating of precured PDMS solution onto the CNT film. We found that due to PDMS infiltration down to the substrate, as-grown CNTs were surrounded by PDMS. In such a composite structure, the thermal energy in the CNTs can be efficiently transferred to the PDMS and subsequently used for volume thermal expansion and ultrasound generation. Particularly, to facilitate the infiltration and formation of a thin layer (several $\mu \mathrm{m}$ ), we used a high-modulus PDMS, which was designed to have a reduced viscosity as compared to those commercially available (e.g., Sylgard 184, not diluted). The high-modulus PDMS also prevents CNTs from forming agglomerations, which can negatively affect the photoacoustic generation. ${ }^{[29]}$ It was found that the CNT morphology plays an important role for both heat transition to the surrounding medium and optical extinction properties. The morphology was controlled by growth conditions that determine the length of CNT strands as well as their areal density on a substrate (Figure 4). For short growth time (usually less than a minute), 

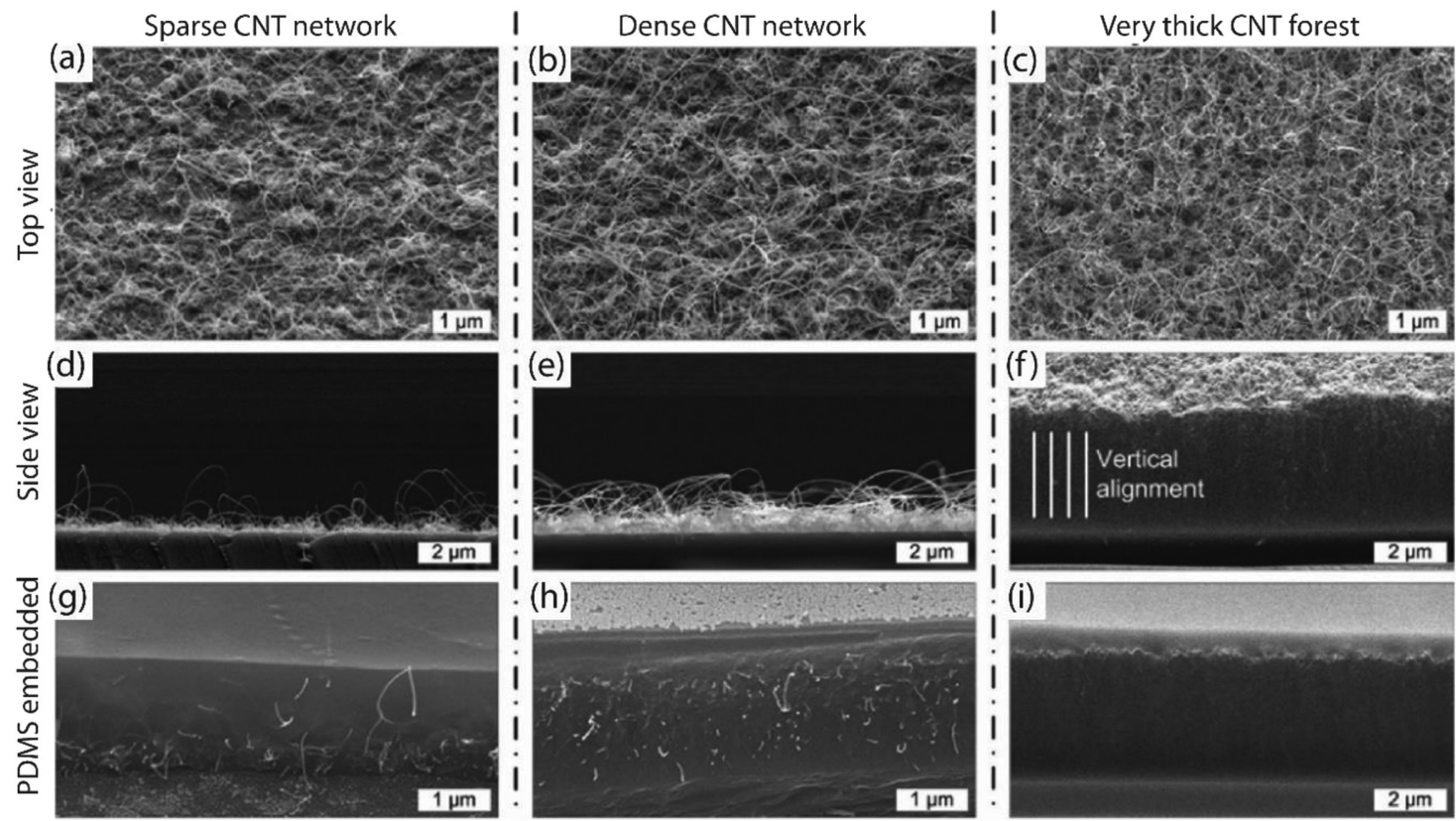

Figure 4. SEM images of the CNT layers. Without PDMS coating, the CNT films for different areal densities: a) sparse, b) dense, and c) thick CNT layers. $d-f$ ) Corresponding cross-sectional views of the CNT films. g-i) The CNT films with PDMS. Reproduced with permission. ${ }^{[29]}$ Copyright 2015, The Royal Society of Chemistry.

the areal density of CNTs was low, thus forming a sparse, tangled CNT network. Although this sparsely grown tangled structure is good for PDMS infiltration, it compromises optical absorption. However, as the growth time increases, the CNT morphology transformed into a densely packed structure with vertical alignment due to a crowding effect of CNTs. Note that the crowded CNT forest can provide a substrate coverage with high areal density. Although this structure allows extremely high optical absorption, such forest structure has too dense to allow the infiltration of PDMS prepolymer liquid. This is a critical drawback of the forest only having a physical interface on the upper end of CNT strands with PDMS. In this arrangement, only the upper ends of the CNTs are in contact with PDMS. Moreover, as the bottom end of CNT is not robustly fixed by PDMS to the substrate, the adhesion of the CNT strands to the substrate is poor, thus susceptible to physical delamination or laser-induced damage. Therefore, the growth time together with other atmospheric and thermal conditions within a CVD chamber should be optimized to obtain a proper CNT length and density, which can allow the PDMS prepolymer liquid to infiltrate and thus form a well-mixed nanocomposite. When individual CNT strands are completely embedded and then surrounded by the PDMS, the nanoscale element can take the advantage of rapidly and efficiently transferring thermal energy to the surrounding PDMS.

As the thick CNT forest structure has limitation to form the well-mixed composite with PDMS, despite its high optical absorption $(\approx 100 \%)$, CNT films were prepared with a laterally tangled network (prior to forming the vertically aligned forest). Such film allowed formation of the nanocomposite with PDMS but had slightly low optical absorption (up to $80 \%$ ). To further increase the light absorption, a gold layer $(20-30 \mathrm{~nm})$ was physically deposited onto an as-grown CNT film, followed by PDMS coating. The additive gold was deposited directly on CNTs increasing optical absorption cross-section and also deposited on the substrate surface in a shadowed manner through the CNT network. The latter gold layer bounds on the substrate produced random nanoclusters, which contributes to the enhancement of optical absorption.

It should be also noted that mechanical robustness of the composite is associated with the complete infiltration of PDMS down to the substrate to provide the nanocomposite with strong adhesion with the substrate. As a result, the film can withstand laser irradiation with an extremely high-pulse energy without physical ablation $\left(>400 \mathrm{~mJ} \mathrm{~cm}{ }^{-2}\right.$ ). For pulsed laser beams $(5-10 \mathrm{~ns}$ temporal width), this value is $7-8$ folds higher than those of other thin-film transmitters directly coated on substrates such as $\mathrm{Cr}$ film and 2D gold nanostructured film. Such high value of laser damage threshold enables CNTs to absorb a high optical energy that is available for photoacoustic generation.

\subsubsection{CNT Solution}

Motivated by the efficient photoacoustic conversion of the asgrown CNT-PDMS composite, Colchester et al. utilized chemically functionalized CNTs mixed with PDMS for a CNT-PDMS composite. Based on a dip-coating technique, a CNT-PDMS transmitter was fabricated on a tiny end face of an optical fiber. This allowed formation of a fiber-based miniature transmitter and then integration with additional ultrasound detectors (e.g., fiber-based Fabry-Perot interferometer (FPI)), offering possibility for intravascular applications. In this approach, MW-CNT powders (6-9 $\mathrm{nm}$ in diameter; $5 \mu \mathrm{m}$ in length) were used for chemical modification to overcome the issue of CNT agglomeration. CNTs modified with functional groups allowed dispersion 
in xylene and formation of a composite with PDMS, avoiding sedimentation. For preparing the solution of a CNT-PDMS composite, they used functionalized CNT mixed with xylene solution. Then, the mixed solution was sonicated and degassed to remove agglomeration and facilitate dispersion process.

The above solution-processed nanocomposite was further improved by Noimark et al., ${ }^{[11]}$ who tried to reduce the composite coating thickness of photoacoustic transmitters for high-frequency broadband ultrasound, which is useful for highresolution biomedical imaging. Because ultrasound attenuation in composites increases dramatically with thickness, minimization of the composite coating thickness can achieve large bandwidth and large pressure amplitudes for high resolution and sensitivity, respectively. They demonstrated methods for making a high concentration MWCNT formulation and an MWCNT organogel, which were used for multiwalled CNTPDMS composites coated on the end faces of optical fibers. From SEM imaging, they observed that the PDMS infiltrated the MWCNT coatings to form composites (Figure 3d).

The MWCNT-organogel was formulated with addition of acetone into high concentrations of MWCNTs. High concentrations of MWCNTs were achieved using the oleylamine functionalized-pyrene ligand. The MWCNT-gel was fabricated by preparing a high concentration of MWCNT solution dispersed in xylene, using oleylamine functionalized pyrene ligand, and then adding acetone for gelation. This structure was then mixed with PDMS for composite formation. They found that the MWCNT-gel coating with optical absorption greater than $97 \%$ had a homogeneous appearance (complete, uniform coverage across the entire fiber end face), compared to MWCNTPDMS integrated coating showing coffee-ring effects. The small coating thicknesses were achieved without compromising the optical absorption of the MWCNT coatings. They confirmed pressure performance by fabricating a fiber-optic transmitter with the MWCNT-gel-PDMS composite, reporting high-amplitude pressure of $21.5 \mathrm{MPa}$ with a broad bandwidth of $29 \mathrm{MHz}$ ( $6 \mathrm{~dB}$ roll-off on the right shoulder).

Although various methods have been used to fabricate optical ultrasound transmitters, thickness control of absorbing layers on an optical fiber end-face can be challenging. For precise control of transmitter thickness, an electrospinning technique was utilized by Poduval et al. ${ }^{[30]}$ The proposed coating method was capable of electrospinning onto an optical fiber end-face, allowing good thickness control as well as high PDMS elastomer infiltration. The absorbing material was made of MWCNTs in polyvinyl alcohol. The electrospinning was followed by dip-coating with PDMS. Nanofibers produced by electrospinning have $\approx 207 \mathrm{~nm}$ diameter, and also have high porosity, permitting high PDMS infiltration. The orientation of MWCNTs are preferentially aligned along the nanofiber axis. In addition, electrospinning allows for accurate control of the coating thickness with a range of 2.3-41.4 $\mu \mathrm{m}$ through electrospinning time, and facilitate optical absorption by creating ideal alignment of MWCNTs with respect to the optical fiber end-face. A uniform nanofibrous mesh of MWCNT-PVA was formed on the fiber surface for controllable formation of CNT-PDMS composite. The mesh played roles of arranging MWCNTs preferentially and providing space for infiltration of PDMS down to the underlying substrate. They compared the electrospun absorbers with that prepared by dip-coating. In comparison, the dip coating had larger coating thicknesses, whereas the absorber by electrospinning produced a much greater ultrasound pressure amplitude as well as a broader bandwidth. This demonstrated that a certain optimal thickness $(13.7 \mu \mathrm{m})$ exists for maximal pressure generation because the composite thickness increases two factors in a trade-off relation simultaneously: optical absorption and internal ultrasound attenuation within the composite coating.

The thickness of photoacoustic composites should be small as long as they still absorb light sufficiently. Horizontally aligned CNTs can be advantageous to realize very thin composite. Instead of using short length CNTs $(<$ several $\mu \mathrm{m})$, Chen et al. used CNT yarns as photoacoustic generation sources. ${ }^{[31]}$ Particularly, these yarns were developed in a multilayered form, exhibiting an extremely high spatial packing density of CNT absorbers. They used a CVD spinning process to produce the continuous yarn of CNTs. Monolayers of CNTs were first fabricated and then concentrically assembled in seamless tubules. Additional gold nanoparticles $(10 \mathrm{~nm})$ were deposited over the CNT yarn to increase photoacoustic conversion efficiency, followed by coating of PDMS to form a composite structure, where PDMS can infiltrate into the horizontally aligned CNT yarn layers. Moreover, the CNT yarn-PDMS composite transmitter could be fabricated on a soft backing layer (i.e., PDMS), unlike other thin-film transmitters mostly formed on hard substrates such as glass.

The CNT yarn-PDMS composite with Au particles showed 1.5 times larger light absorption than that without Au nanoparticles, thus increasing the peak positive pressure amplitude to 33.6 MPa at a laser fluence of $45 \mathrm{~mJ} \mathrm{~cm}{ }^{-2}$, compared to the pure CNT yarn-PDMS of $19 \mathrm{MPa}$. The light absorption enhancement due to the Au particles was explained with localized surface plasmon resonance (LSPR) and consequent increase in their effective size, which resulted in significant heating of the $\mathrm{Au}$ particles as well as the surrounding medium. The photoacoustic conversion efficiency of the composite with Au was calculated to be $2.74 \times 10^{-2}$. The photoacoustic amplitudes were high enough to manipulate microparticles $(50 \mu \mathrm{m})$, and they showed that the microparticles near the photoacoustic film were translated larger than $15 \mathrm{~mm}$. This prove that acoustic radiation forces induced by short photoacoustic pulses were capable of moving liquids and particles over a long distance in a controlled manner. This approach is potentially useful for drug delivery applications.

\subsubsection{Other Carbon Materials}

Carbon Black: Since Alexander Graham Bell used lampblack (kind of carbon black) for the photoacoustic effect a century ago, carbon black has been widely used for photoacoustic generation. Later, Biagi et al. fabricated a composite consisting of epoxy resin mixed with graphite powder. ${ }^{[32]}$ They observed that photoacoustic signals were very high compared to those generated with a control sample (an aluminum layer of $100 \mathrm{~nm}$ thickness). By using PDMS instead of epoxy, Buma et al. fabricated a CB-PDMS composite for a photoacoustic array, so as to address the fabrication challenges of piezoelectric 
array transducers operating at frequencies $>50 \mathrm{MHz}{ }^{[14]}$ The addition of carbon black (or graphite) resulted in a highly absorbing PDMS composite. By using the black PDMS film, they reported a significant increase in photoacoustic amplitude, compared to metal films. Such enhancement was explained by facilitated heat transfer between the carbon black to the elastomer (although the size of CBs was not informed in their work). In addition to the high amplitude, the CBPDMS film excited by pulsed laser irradiation (10 ns) was reported to produce a high-frequency pulse with a bandwidth of $\approx 50 \mathrm{MHz}$.

Candle Soot Nanoparticle Composite: Carbon black nanoparticles can be produced through a simple process where a candle (paraffin wax) is burned. Such carbon nanoparticles (or soot) are known to have a porous structure, showing high optical absorption. To use the high optical absorption of the carbon nanoparticles, Chang et al. fabricated a photoacoustic transmitter. ${ }^{[8]}$ This process to make carbon nanoparticles is also known as flame synthesis. ${ }^{[33]}$ Using the carbon nanoparticles they fabricated a candle soot nanoparticle (CSNP)-PDMS composite. Since the carbon soot nanoparticles deposited on a substrate are washed out by spin coating of PDMS, they used a direct transfer process, where an uncured PDMS layer (prepared by spin-coating) on a substrate was put in contact of the CS layer coated on a glass substrate. The direct transfer of the PDMS solution did not only preserve the CS layer, but also it allows the PDMS solution to penetrate the porous CS layer. Once the PDMS solution completely penetrated, the mixture of the CS layer and PDMS solution was fully cured. The SEM image (taken before PDMS transfer) of the CSNPs showed nanoparticles of $\approx 50 \mathrm{~nm}$ (Figure $3 \mathrm{~b}$ ). Notably, they found that the gentle transfer enabled a thin composite $(<30 \mu \mathrm{m})$ consisting of a PDMS-only layer $(\approx 20 \mu \mathrm{m})$ and the mixed layer $(\approx 6 \mu \mathrm{m})$. Indeed, the porous composite showed the high light absorption $(\approx 96 \%)$. Using a pulsed laser (6 ns), they reported high-pressure

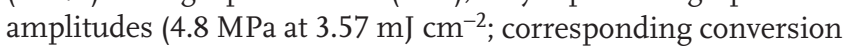
efficiency of $4.41 \times 10^{-3}$ ) owing to the high light absorption and small thickness of the composite. They explained that the high performance resulted from the efficient heat transfer from the carbon nanoparticles to the surrounding PDMS that is enabled by the maximized surface area in the nanoparticles (surface-tovolume ratio)

Carbon Nanofiber Composite: CNFs share a common feature with CNTs; they have a cylindrical nanostructure, showing high light absorption and thermal conductivity. ${ }^{[34]}$ Also, CNFs can be easily fabricated with a low-cost material, making an efficient photoacoustic composite. Hsieh et al. produced a CNFs-based composite. The CNFs was prepared by electrospinning of the mixture of Polyacrylonitrile and N,N-Dimethylformamide, followed by carbonization at several hundreds of temperature. ${ }^{[9]}$ They found that CNF strands were sufficiently dense (strands' diameter of $\approx 130 \mathrm{~nm}$ ) (Figure $3 \mathrm{c}$ ). PDMS solution was spincoated on a CNF layer, forming a CNFs-PDMS composite with $\approx 95 \%$ optical absorption and pressure amplitude $(12 \mathrm{MPa}$ at $3.71 \mathrm{~mJ} \mathrm{~cm}^{-2}$ ).

Reduced Graphene Oxide: For more than a decade, graphene has received great attention because of excellent physical properties in terms of electronic, thermal, optical, and mechanical aspects. One of the graphene derivatives - rGO was introduced as a photoacoustic transmitter due to its high optical absorption and nanoscale dimension. Lee et al. proposed an rGObased thin-film transmitter that has an rGO layer coated with aluminum (<200 nm thickness). ${ }^{[15]}$ First, they prepared GO, synthesized from graphite flakes, as dispersed in water to make a GO solution. The combined layer of $\mathrm{rGO}$ and aluminum worked as a light absorber and a thermoacoustic source for pressure generation. ${ }^{[12]}$ Later, the rGO-based structure was improved by incorporating black carbon-doped PDMS instead of the aluminum coating. By taking the advantage of high thermal expansion of PDMS and enhanced optical absorption by the black carbon, an output pressure amplitude could be increased 76 times higher than the one generated by the aluminum coating alone (detector bandwidth: 0.25-10 $\mathrm{MHz}$ ). The rGO-based transmitters demonstrated the importance of the adjacent heat transfer medium to achieve high-pressure generation.

\subsection{Metal-Based Materials for Resonance Absorption}

\subsubsection{Nanostructured Gold Array}

To increase a photoacoustic frequency bandwidth, the thickness of a photoacoustic composite should be as small as possible. Hou et. al used a thin composite consisting of gold nanostructure and polymer. ${ }^{[6]}$ First, they coated gold nanoparticles on a transparent substrate, which was then covered with a PDMS layer. They used diluted PDMS solution for a thinner PDMS layer, compared to that produced by a high viscosity of PDMS solution mixed with particles. ${ }^{[14]}$ Here, the gold nanoparticles were fabricated by a nanofabrication technique (Figure 5a). They found that the gold array exhibited peak absorption at resonance due to localized surface plasmons around the particles. Although the absorption of the gold composite was not that high, photoacoustic signals were measured to be comparable to that of a carbon-based PDMS composite. However, compared to the carbon-based PDMS composite, the gold composite showed a considerable enhancement especially for high frequencies due to its thickness. Also, they explained that this enhancement resulted from fast thermal energy transfer (about 100 fs time duration) between the absorbing layer and PDMS. They also fabricated a 3D structure for increasing light absorption (Figure 5c). ${ }^{[35]}$

\subsubsection{Gold Nanoparticle PDMS Composite}

Photoacoustic conversion was significantly increased by the nanostructured gold array (layered structure). ${ }^{[6,35]}$ However, the main problem of such a layered structure was relatively low light absorption because of its small thickness. To increase light absorption by using optical resonance, Wu et al. fabricated a PDMS-gold nanoparticle composite for photoacoustic generation. ${ }^{[36]}$ Instead of mixing of $\mathrm{Au}$ NPs, the composite was fabricated by one-pot synthesizing gold particles within PDMS. This fabrication method can address some challenges involved in conventional approaches such as spin-coating or mixing (e.g., agglomeration of NPs, and poor infiltration) while 
(a)

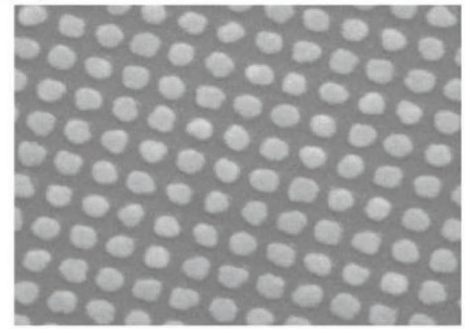

(c)

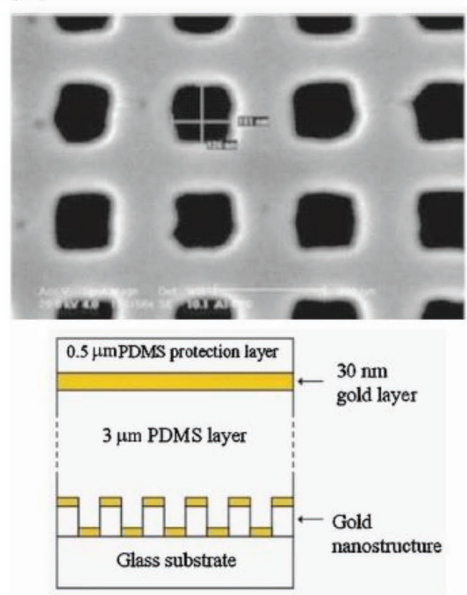

(b)

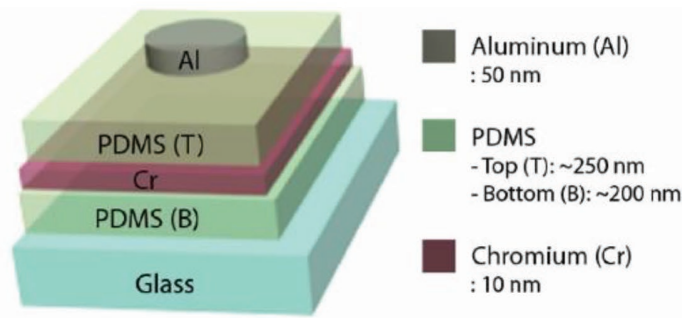

(d)

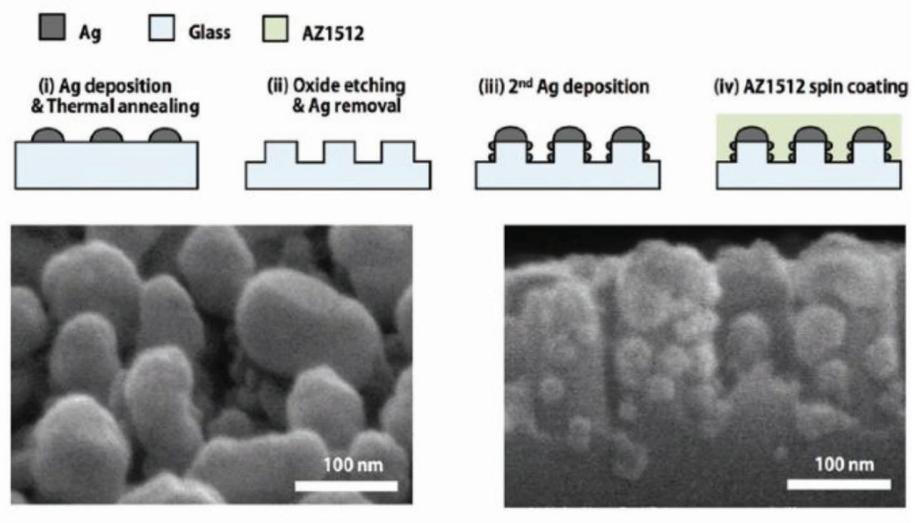

Figure 5. Metal-based photoacoustic materials. a) Gold structure (2D) on a glass substrate. Adapted with permission. ${ }^{[6]}$ Copyright 2016, AIP Publishing LLC. b) Thin metal film-PDMS composite. Adapted with permission. ${ }^{39]}$ Copyright 2016, Wiley-VCH. c) Gold structure (3D) (top: SEM image; bottom: (side view) schematic with dimensions). Adapted with permission. ${ }^{[35]}$ Copyright 2007, AIP Publishing LLC. d) Glass nanopillar arrays with silver islands. Adapted with permission. ${ }^{[37]}$ Copyright 2016, The Royal Society of Chemistry.

increasing the concentration of gold nanoparticles for high light absorption. They observed that the size of Au NPs was around $<30 \mathrm{~nm}$. They optimized the concentration (1.79 $\mathrm{wt} \%)$ and thickness $(450 \mu \mathrm{m})$ of the nanocomposite film. The pres-

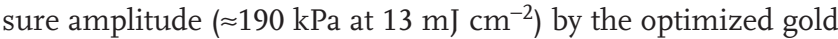
composite was observed to be orders of magnitude higher than a thin metal film.

\subsubsection{Silver Structure}

Conventional photoacoustic composites usually require efficient heat transfer from light absorbers to the surrounding materials for increasing photoacoustic conversion. In other word, such approach may be not effective when the heat capacity of light absorbers is high. Alternatively, a phenomenon so-called EM hotspots, supporting local field enhancement around nanostructures, does not require thermal transfer between optical absorbers and thermal expansion media. Park et al. fabricated metallic nanostructures supporting LSPR. ${ }^{[3]}$ By using this approach, optical energy is deposited to the surrounding medium instead of transferring from the metal structure. They produced glass nanopillar arrays coated with an optical absorbing polymer (AZ1512) (Figure 5d). They found that the structure showed a significant improvement, compared to a control sample (i.e., polymeric absorber).

\subsubsection{Metal Film/Polymer Composites}

Bare metal films have been widely used for photoacoustic generation due to easy fabrication. However, the thermal expansion of these metals is relatively low. To mimic the approach used for carbon-based composites, polymer layers were coated on a metal layer. ${ }^{[38]}$ Kang et al. investigated the thermoelastic proximity effect of metallic layers by using different polymer materials. ${ }^{[38]}$ They found that photoacoustic amplitudes produced were varied depending on these polymer materials. Specifically, they found that replacing parylene with PDMS led to a considerable increase in the peak pressure amplitude. Although the bilayer metal composites showed high photoacoustic conversion efficiency compared to a metal layer alone, their photoacoustic conversion was lower than carbon-based composites.

To demonstrate an efficient metal-based photoacoustic transmitter, we used a thin metal film sandwiched by polymer layers, as illustrated in Figure $5 b{ }^{[39]}$ At first, we tried to maximize the heat transfer from the metal film to the surrounding polymers, because the photoacoustic conversion in the metal film is not efficient and the thermal energy stored in the metal film is just wasted. A metal film (thickness $10 \mathrm{~nm}$ ) was used, and it has low heat capacity compared to thick metal films, thereby facilitating heat transfer to the polymer layers. The heat energy transferred to the polymers was calculated, which was observed to be $>70 \%$ of the absorbed optical energy. In contrast, 
the $100 \mathrm{~nm}$ thick metal films deliver only $10 \%$ heat energy to the surrounding layers. Moreover, it was observed the negative impact of transparent substrates (e.g., glass) used for the metal film coating. When the metal film is in a direct contact with the glass substrate, significant heat energy in the metal film is transferred into the glass substrate. By placing a polymer layer between the substrate and the metal absorber, significant heat energy, which otherwise get wasted, was used for photoacoustic conversion.

Although the ultrathin film in this study showed better performance from a heat transfer perspective, it exhibited poor optical absorption. The metal film was too thin to effectively absorb light for photoacoustic generation. This issue was addressed by using a resonance cavity, which was realized by adding an additional metal layer as an optical reflector, showing $90 \%$ of light absorption in the metal film. ${ }^{[39]}$ The photoacoustic conversion of the metal film composite was comparable to carbon-based polymer composites (e.g., CNTPDMS composites). This approach does not only offer an effective way to increase photoacoustic amplitudes in metal films, but also such simplified platform allows a better understanding of photoacoustic generation by providing an analytical formula describing heat transfer between absorbers and the surrounding mediums.

\subsubsection{Nanostructured Ge-PDMS Composite}

In an effort to form nanostructured light-absorbing transmitters, Yoo et al. used germanium (Ge) films deposited on quartz substrates. ${ }^{[40]}$ Ge films of different thicknesses $(300,500$, and $700 \mathrm{~nm}$ ) were first deposited by an electron-beam evaporation process, and then solution-dispersed gold nanoparticles were spin-coated over the films. Then, a metal assisted chemical (MAC) etching process was used by simply embedding the Ge films in deionized water, resulting in nanostructured Ge films. In this process, the oxygen dissolved the water medium led to reduction and oxidation of the Ge surface. Then, as the Ge oxide $\left(\mathrm{GeO}_{2}\right)$ was dissolved in water, the Ge nanostructure could be finally formed only around the deposited gold nanoparticles. The Ge-nanostructured transmitter generated 7.5-fold higher pressure (with a frequency bandwidth of $\approx 27 \mathrm{MHz}$ ) than a $\mathrm{Cr}$ film (100 nm thick) used as a reference sample. Although the output pressure amplitude corresponded to around $42 \%$ level of CNT-PDMS composite ( $4.5 \mu \mathrm{m}$ thick), this approach is significant in terms of easy and reliable fabrication of nanostructured transmitters, and it also control absorber thickness accurately by the evaporation process.

\subsection{Figure of Merit and Performance Comparison}

\subsubsection{Figure of Merit}

For efficient photoacoustic conversion, both good light absorption and large thermal expansion coefficient are important. For the design of photoacoustic composites consisting of lightabsorbing fillers combined with transparent polymers, the light absorption performance of these fillers reaches near unity. Therefore, the photoacoustic conversion is fundamentally limited by the thermal expansion coefficients of the transparent polymers. In this regard, PDMS is the most widely used, as shown in the previous section. ${ }^{[7,9,10,14]}$

An important question is whether PDMS is really the best choice for photoacoustic generation. To answer this question, one can recall Equation (7), which states that the photoacoustic amplitudes are not only proportional to the thermal expansion coefficient, but also other material properties, i.e., the Grüneisen parameters. Thus, it is reasonable to consider that the Grüneisen parameter is the measure of photoacoustic conversion. Table 1 summarizes the material properties used for calculating photoacoustic amplitudes. Interestingly, in terms of the Grüneisen parameters, PDMS is not as good as gold (Au), i.e., $\left(\Gamma_{\mathrm{Au}} / \Gamma_{\mathrm{PDMS}} \approx 5\right)$. Also, most of metals are better than PDMS.

Before concluding the question, one should also consider photoacoustic transmission. In practice, photoacoustic waves travel across interfaces between different mediums. If these mediums have different acoustic impedances, some of photoacoustic energy is reflected at the interface and the rest is transmitted. The acoustic transmission $\left(T_{12}\right)$ from the medium 1 to the medium 2 is given by

$T_{12}=\frac{2 Z_{2}}{Z_{1}+Z_{2}}$

where $Z$ is the acoustic impedance $(Z=\rho c)$ and the subscripts 1 and 2 correspond to the medium 1 and medium 2. For example, photoacoustic signals are typically measured in water, and thus the photoacoustic generation of materials should be compared based on the measured ones by considering acoustic transmission into water. When photoacoustic transmission into water $\left(Z_{2}=Z_{\mathrm{w}}\right)$ is considered, one can modify Equation (12) as

$T_{\mathrm{w}}=\frac{2 Z_{\mathrm{w}}}{Z+Z_{\mathrm{w}}}=\frac{2}{Z / Z_{\mathrm{w}}+1}$

Thus, Figure of merit for photoacoustic generation can be defined as

$\mathrm{FOM}=\Gamma T_{\mathrm{w}}$

Table 1. Material properties.

\begin{tabular}{lcccc}
\hline Material & $\begin{array}{c}\text { Sound speed }(c) \\
{\left[\mathrm{m} \mathrm{s}^{-1}\right]}\end{array}$ & $\begin{array}{c}\text { Acoustic } \\
\text { impedance }\left(Z_{w}\right)^{\mathrm{a})}\end{array}$ & $\begin{array}{c}\text { Grüneisen parameter } \\
\left(\Gamma=\frac{\beta c^{2}}{C_{\mathrm{p}}}\right) \times 10^{-6}\end{array}$ & $\begin{array}{c}\text { Figure of } \\
\text { Merit } \Gamma T_{\mathrm{w}} / \Gamma_{\mathrm{w}}\end{array}$ \\
\hline Water & 1500 & 1 & 0.111 & 1 \\
Gold (Au) & 3240 & 41.7 & 3.391 & 1.43 \\
Titanium(Ti) & 6070 & 18.2 & 1.817 & 1.71 \\
Chrome (Cr) & 5940 & 28.5 & 1.426 & 0.87 \\
Fused silica & 5968 & 8.8 & 0.033 & 0.06 \\
PDMS & 987 & 0.7 & 0.620 & 6.57 \\
Parylene & 2200 & 1.9 & 0.713 & 4.43 \\
\hline
\end{tabular}

a) Acoustic impedances are normalized to that of water $\left(Z_{w}\right)$. 
As seen in Table 1, FOM of PDMS, normalized to the Grüneisen parameter of water $\Gamma_{\mathrm{w}}$, is better than the other materials listed.

\subsubsection{Theoretical Limit}

The photoacoustic conversion efficiency, defined by a ratio of output acoustic energy $\left(E_{\text {aco }}=S \int_{-\infty}^{\infty} \frac{P^{2}(t)}{\rho c} \mathrm{~d} t\right)$ to input optical energy $\left(E_{\mathrm{opt}}=F \cdot S\right)$, is represented by

$\eta=\frac{E_{\text {aco }}}{E_{\text {opt }}}=\frac{E_{\text {aco }}}{F S}=\frac{1}{F} \int_{-\infty}^{\infty} \frac{P^{2}(t)}{\rho c} \mathrm{~d} t$

For efficient photoacoustic conversion, as indicated in Equation (5), the absorbing layer thickness (acoustic source length) should be much smaller than the acoustic propagation length during the laser pulse duration, as long as the thin layer does not significantly compromise light absorption. Thin light-absorbing layers satisfies the requirements for the long pulse regime. Moreover, photoacoustic generation in conventional polymer composites is mostly classified as the long pulse regime. Thus, the theoretical limit derived in the long pulse regime could be useful.

In the long pulse regime, the temporal profile of photoacoustic pulses is equal to that of Gaussian laser pulses such that $P(t)$ is expressed with the pulse duration of the laser pulses $\left(\tau_{l}\right)$, i.e., $P(t)=P_{0} \exp \left(-\ln 2 \frac{t^{2}}{\tau_{l}^{2}}\right)$. From Equation (15) with $\int_{-\infty}^{\infty} P^{2}(t) \mathrm{d} t=0.75 P_{0}^{2} \tau_{l}$ and $P_{0}=\Gamma \cdot A \cdot \frac{F}{c \tau_{l}}$, the photoacoustic conversion efficiency in the long pulse regime can be determined by $\eta=0.75 \cdot \frac{P_{0}^{2} \tau_{l}}{\rho c F}=0.75 \frac{\Gamma^{2} A^{2}}{\rho c^{3} \tau_{l}} F$

By plugging $\Gamma=\frac{\beta c^{2}}{C_{\mathrm{p}}}$ into Equation (16), $\eta=0.75 A^{2}\left(\frac{\beta}{C_{\mathrm{p}}}\right)^{2} \frac{c F}{\rho \tau_{l}} .{ }^{[39]}$ For polymer composites, the photoacoustic amplitude is mainly determined by the thermal energy delivered to the constituent polymers (i.e., $\left.P_{0} \sim \gamma_{\mathrm{p}} A F\right)$. Thus, the photoacoustic conversion efficiency is proportional to the laser fluence $\eta \sim\left(A \gamma_{\mathrm{p}}\right)^{2} F$, indicating that the higher laser fluence, the higher conversion efficiency. The linear relationship between the laser fluence and conversion efficiency is also confirmed by a more rigorous derivation. ${ }^{[41]}$ However, the laser fluence cannot be too high and is constrained with the allowable temperature $\left(\Delta T_{\mathrm{a}}\right)$ of the heated material, $F<\left(\rho l_{\mathrm{th}} C_{\mathrm{p}}\right) \Delta T_{\mathrm{a}}$ with $l_{\mathrm{th}}=\sqrt{\alpha_{\mathrm{th}} \tau_{l}}$ the heat diffusion length. Here, the allowable temperature increase $\left(\Delta T_{a}\right)$ is determined with material damage. Also, it should be noted that a considerable temperature increase can change the material properties. For efficient PDMS-based composites in the ideal conditions $\left(A=1, \gamma_{p}=1\right)$, Equation (16) with $\tau_{l}=6 \mathrm{~ns}$ is calculated to be $\eta=4.9 \times 10^{-5} \mathrm{~F}$. With an assumed material-damage threshold

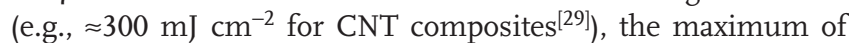
$F=300 \mathrm{~mJ} \mathrm{~cm}{ }^{-2}$ can lead to a photoacoustic conversion efficiency of $\eta=0.147$ (14.7\%). Interestingly, the large portion of the input energy is used to increase the temperature.

\subsubsection{Performance Comparison}

Table 2 summarizes the photoacoustic generation performance of composite materials, which are characterized with peak pressure amplitude, bandwidth, and energy conversion efficiency. Because the photoacoustic performance was measured using

Table 2. Performance comparison. To compare performance obtained different laser fluence and pulse duration, one can define $C F=\left(\frac{P_{0}}{F}\right)\left(\frac{\tau_{1}}{6 n s}\right)$.

\begin{tabular}{|c|c|c|c|c|c|c|c|}
\hline & Composites & Pressure $\left(P_{0}\right)$ & Laser fluence $(F)$ & $\begin{array}{l}\text { Laser pulse } \\
\text { duration }\left(\tau_{l}\right)\end{array}$ & $C F$ & Coating thickness & Remark \\
\hline \multirow[t]{8}{*}{ Carbon materials } & Graphite-epoxy ${ }^{[32]}$ & $20 \mathrm{kPa}$ & $2.47 \mathrm{~mJ} \mathrm{~cm}^{-2}$ & & & & $30 \mathrm{MHz}(-3 \mathrm{~dB})$ \\
\hline & CB-PDMS ${ }^{[14]}$ & $\begin{array}{l}20 \mathrm{~dB} \text { enhancement } \\
\text { compared to metal }\end{array}$ & & & & & \\
\hline & Candle soot ${ }^{[8]}$ & $4.8 \mathrm{MPa}$ & $3.57 \mathrm{~mJ} \mathrm{~cm}-2$ & $6 \mathrm{~ns}$ & 1.34 & $18 \mu \mathrm{m}$ & \\
\hline & CNT-PDMS[7,29] & $18 \times \mathrm{Cr}$ & $3 \mathrm{~mW}$ & $6 \mathrm{~ns}$ & & & $>100 \mathrm{MHz}(-6 \mathrm{~dB})$ \\
\hline & Solution $\mathrm{CNT}^{[10]}$ & $4.5 \mathrm{MPa}$ & $34.6 \mathrm{~mJ} \mathrm{~cm}{ }^{-2}$ & $2 \mathrm{~ns}$ & & $20 \mu \mathrm{m}$ & $2 \mathrm{~mm}$ away \\
\hline & CNT-ge|[11]] & $21.5 \mathrm{MPa}$ & $33.1 \mathrm{~mJ} \mathrm{~cm}^{-2}$ & $2 \mathrm{~ns}$ & 0.22 & & \\
\hline & Carbon fiber ${ }^{[9]}$ & $2.4 \mathrm{MPa}$ & $3.57 \mathrm{~mJ} \mathrm{~cm}^{-2}$ & $6 \mathrm{~ns}$ & 0.67 & & \\
\hline & $\mathrm{rGO}^{[15]}$ & $10 \mathrm{MPa}$ & $56 \mathrm{~mJ} \mathrm{~cm}^{-2}$ & $5 \mathrm{~ns}$ & & & \\
\hline \multirow[t]{6}{*}{ Metal-based } & 2D gold ${ }^{[6]}$ & $1.5 \mathrm{MPa}$ & $0.2 \mathrm{~mJ} \mathrm{~cm} \mathrm{~cm}^{-2}$ & $5 \mathrm{~ns}$ & $6.25^{\mathrm{a})}$ & & \\
\hline & $3 \mathrm{D}$ gold $^{[35]}$ & $10 \mathrm{~dB}$ SNR & $26 \mathrm{~mJ} \mathrm{~cm} \mathrm{~cm}^{-2}$ & $5 \mathrm{~ns}$ & & & $>57 \mathrm{MHz}(-6 \mathrm{~dB})$ \\
\hline & Silver pillar ${ }^{[37]}$ & 25 times AZ1512 alone & - & $5 \mathrm{~ns}$ & & & $>30 \mathrm{MHz}(-6 \mathrm{~dB})$ \\
\hline & Gold NPs-PDMS[36] & $189.49 \mathrm{kPa}$ & $13 \mathrm{~m} / \mathrm{cm}^{-2}$ & $150 \mathrm{~ns}$ & 0.33 & & 3.1 $\mathrm{MHz}(-3 \mathrm{~dB})$ \\
\hline & Thin metal film-polymer ${ }^{[38]}$ & $3.86 \mathrm{MPa}$ & $20 \mathrm{~mJ} \mathrm{~cm}^{-2}$ & $5 \mathrm{~ns}$ & 0.16 & & \\
\hline & Ultrathin metal film-PDMS[39] & $1.82 \mathrm{MPa}$ & $2.35 \mathrm{~mJ} \mathrm{~cm}^{-2}$ & $6 \mathrm{~ns}$ & 0.77 & $<1 \mu \mathrm{m}$ & \\
\hline
\end{tabular}

a) Would need further information. 
(a)

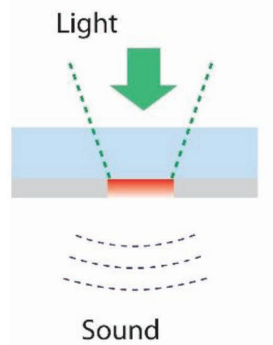

(b)

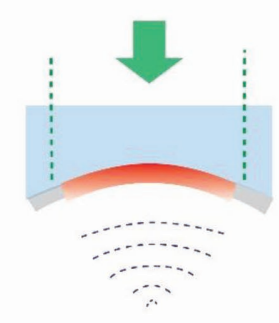

(c)

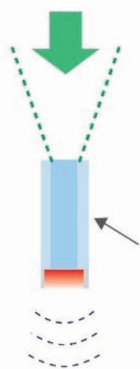

(d)

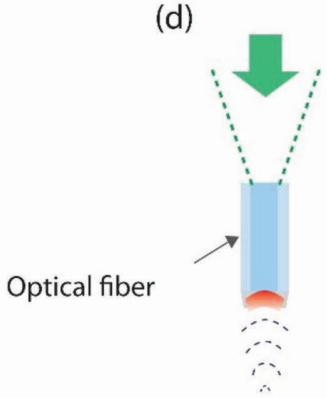

Figure 6. Photoacoustic transmitters. a) Planar transmitters consisting of a thin photoacoustic layer on a transparent substrate. b) Focused transmitters composed of a thin photoacoustic layer coated on a transparent concave substrate. c) Planar fiber-optic photoacoustic transmitters with a photoacoustic coating on the tip of an optical fiber. d) Focused fiber-optic photoacoustic transmitters having a photoacoustic layer coated on the curved transparent surface attached to the tip of an optical fiber.

different settings, care should be taken for fair comparison. When comparing the performance, one should consider the conditions stated below.

1) Peak Pressure Amplitude: The laser pulse duration should be same. If not, for comparison, one would need to use peak pressure amplitudes $\times$ laser pulse duration. Also, for pair comparison, one may need to consider the distance between photoacoustic sources and detectors. The measured pressure signals can be distorted by nonlinear effects (e.g., shock formation) if the distance from the source to the detector is considerably far and the pressure amplitudes are high.

2) Photoacoustic Conversion Efficiency: From Equation (15), the conversion efficiency $(\eta)$ is proportional to the laser fluence $(F)$. When comparing the conversion efficiency between photoacoustic composites obtained by using different $F$, one should use $\eta / F$ instead of $\eta$.

\section{Photoacoustic Transmitters for Novel Applications}

Photoacoustic films are used to construct different types of photoacoustic transmitters depending on applications, as illustrated in Figure 6. Planar transmitters, consisting of a thin photoacoustic layer coated on a transparent glass substrate, are most widely made (Figure 6a) because of easy fabrication, e.g., using spin-coating. To produce high-amplitude photoacoustic pulses, one can use focusing transmitters composed of a photoacoustic film coated on a transparent concave substrate (Figure 6b). ${ }^{[12]}$ Concave substrates for focusing transmitters can be purchased or printed through $3 \mathrm{D}$ printing. Although it is challenging to deposit a thin photoacoustic layer uniformly over a curved surface, there existed successful demonstrations. ${ }^{[12,42,43]}$ In these focused transmitters, generated photoacoustic pulses leaving from the concave surface are concentrated on the center of the radius-of-curvature of the concave surface (focus), having very high-pressure amplitudes, compared to planar transmitters. The pressure amplitudes of focused waves are estimated with multiplication of a focal gain with pressure amplitudes of the identical planar films. Higher focal gains for high level of focusing can be obtained with higher ultrasound frequencies and lower $f$-number (defined as $r / D$ with the radius-of-curvature $(r)$ and diameter $(D)$ of the photoacoustic film-coated concave surface).

Owing to efficient photoacoustic generation in recently developed nanocomposites, one can realize a highly miniaturized flexible photoacoustic transmitter, since high-amplitude photoacoustic signals can be obtained with a small area of photoacoustic composites, e.g., a composite on an optical fiber (diameters smaller than $1 \mathrm{~mm}$ ) (Figure 6c). Such miniaturized photoacoustic transmitter is desirable for imaging and therapeutic applications, e.g., minimally invasive sensing applications. However, it is challenging to create and precisely deposit uniform composite coatings onto optical fibers. Several methods have been demonstrated. For example, the photoacoustic layer on the tip of an optical fiber is prepared through a dip coating method. To apply optical fiber-based transmitters for therapeutic applications, pressure amplitudes are very high (e.g., tens of $\mathrm{MPa}$ for cavitation therapy). One can integrate optical fiberbased transmitters with a focusing scheme (Figure 6d).

\subsection{High-Amplitude Photoacoustic Pulse for Strong, Localized Mechanical Effects}

Because of the characteristics of photoacoustic signals in nanocomposites, many applications have been realized. Particularly, photoacoustic signals generated in the composites have the characteristics of high-amplitude, wide bandwidth, enabling therapeutic and imaging applications. In addition, photoacoustic effects were used to detect THz light pulses. By using efficient photoacoustic nanocomposites, together with focusing configuration, one can generate unprecedentedly strong ultrasound, which are enough to produce cavitation in water. Due to its high frequency, focused ultrasound has highly localized such cavitation.

\subsubsection{High-Precision Cavitation Therapy}

We have demonstrated a CNT photoacoustic transmitter for high-precision targeted therapy. ${ }^{[12]}$ The photoacoustic transmitter consists of a transparent concave lens with a CNT-PDMS 


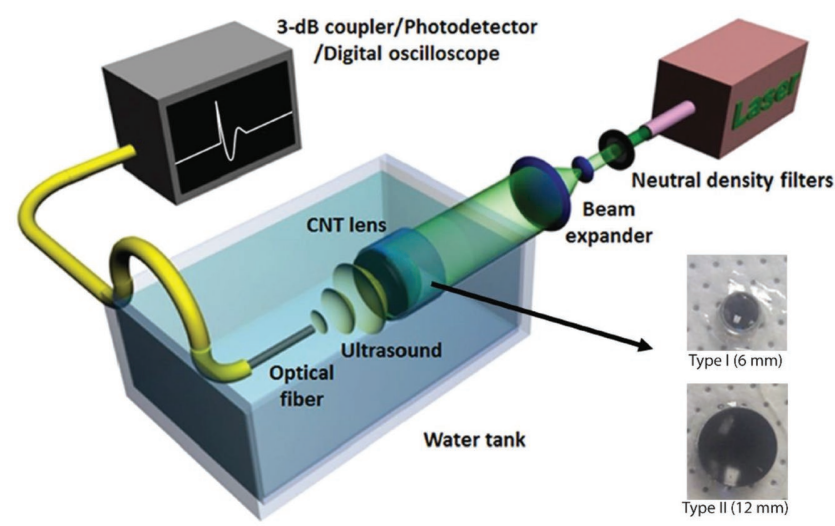

Figure 7. Photoacoustic lenses and measurement setup. The setup consists of the CNT lens (types I, II; different diameters) irradiated by expanded laser beam. The LGFU is characterized by a fiber-optic hydrophone. Adapted with permission. ${ }^{[12]}$ Copyright 2012, Springer Nature.

composite coating, namely PA lens (Figure 7). Upon pulsed excitation of the composite, high-frequency photoacoustic pulses $(>15 \mathrm{MHz}$ ) leaving from the concave surface were focused on a micrometer spot. We observed that the laser-generated focused ultrasound (LGFU) enabled high amplitudes $(>50 \mathrm{MPa})$, as its focal gain was predicted to be $>50$ at $15 \mathrm{MHz}$. Such strong photoacoustic pulses produced microcavitation. Using mechanical effects induced by cavitation and strong pressure pulse, we demonstrated a capability to fragment model kidney stone, and then realized cell-level surgery by selectively removing cancer cells. Microcavitation produced by LGFU was further examined in our lab. ${ }^{[4]}$ In our separate study, we found that LGFU-induced microcavitation was very controllable and deterministic, which was capable of localizing cavitation effect.

The interaction between LFGU and single cell was investigated in detail by Baac et al. in work, ${ }^{[45,46]}$ where transmembrane molecular delivery (Figure 8) and cell detachment (Figure 9) were demonstrated. Figure 8 shows that
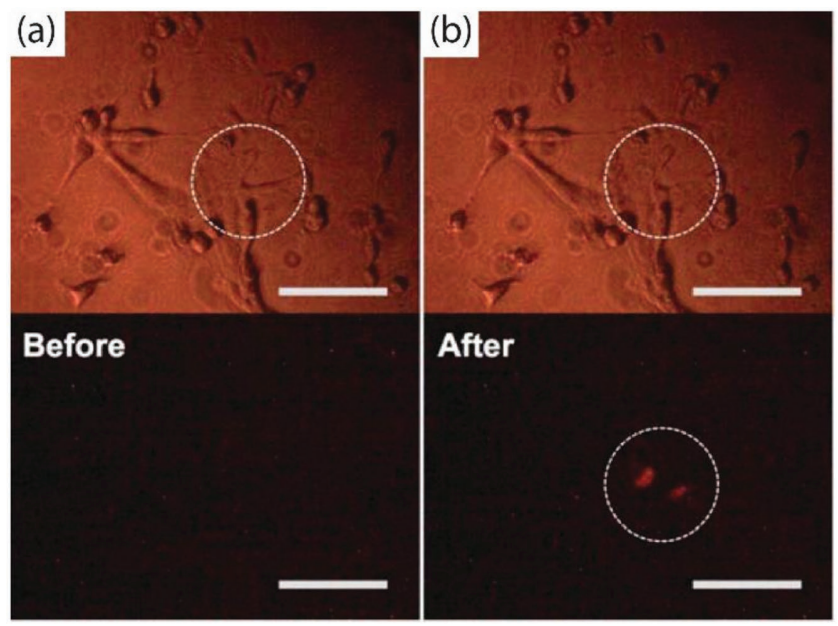

Figure 8. Biomolecule delivery by LGFU (200 pulses). The images a) before and b) after LGFU (top: bright-field images; bottom: fluorescence). Without any change in cell morphology, PI entry (red in the bottom image) was observed. Adapted with permission. ${ }^{[45]}$ Copyright 2013, Wiley-VCH.
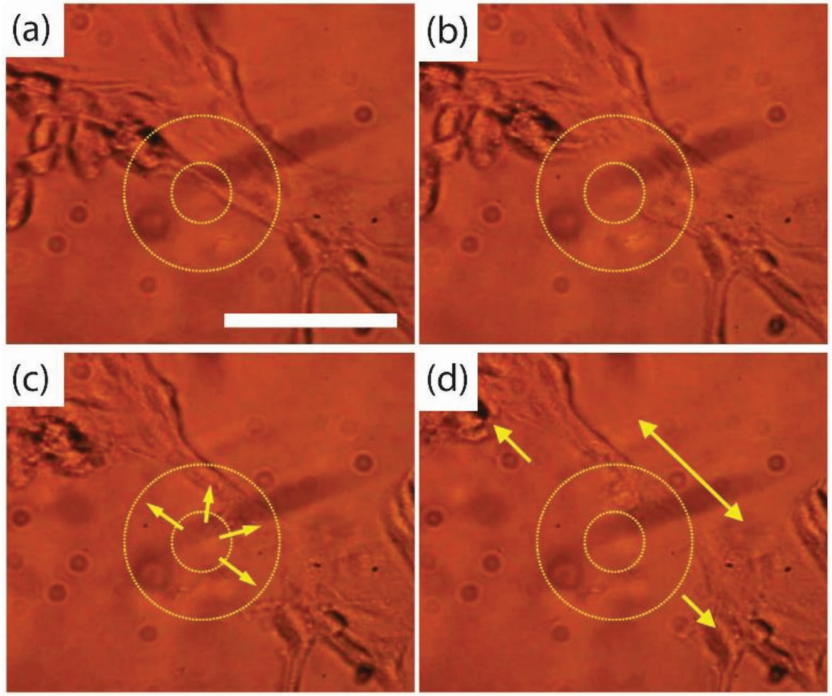

Figure 9. Microfractionation process (scale bar $=100 \mu \mathrm{m}$ ). a) The intact cell-cell junction; b) sharp cut of the junction by LGFU; c,d) The peripheral disruption effects by LGFU. The arrows indicate the radial push of the cells (shown in (c)), and their retreatment (shown in (d)). Adapted with permission. ${ }^{[46]}$ Copyright 2013, OSA.

cavitation-induced microscale disturbance can induce cellular membrane opening and then molecular delivery, using cultured cells. Molecular penetration across the membrane was confirmed by using propidium iodides that work as a membraneimpermeable nucleic-acid binding dye. These were normally blocked and separated by the cell membrane. However, once delivered to the intracellular region of cells, then these bind with DNA and RNA, generating strong fluorescence signal. In this study, only a few cells were targeted by LGFU, and cavitation-induced soft disturbance was used. The soft disturbance by LGFU was achieved at the near-threshold regime for cavitation. Such regime allowed only one cavitation bubble to form on the membranes of a few targeted cells that are placed within the $100 \mu \mathrm{m}$ focal width of LGFU. Cavitational collapse by the single bubble could induce nonthermal perturbation due to liquid jetting and then the trans-membrane delivery of the PI molecules with negligible change in the cell culture morphology. When stronger LGFU amplitudes were used at the overthreshold regime for cavitation $(20 \%$ higher than the threshold level), the PI penetration process was still achieved. However, the cell morphology changed significantly as the cells detached at the center of LGFU focal zone.

The deformation of cultured cellular morphology was further investigated and shown in Figure 9. Here, cavitational disturbance was produced by LGFU with a twofold higher laser energy than the threshold level. This could be first used to sharply cut a single junction connecting two cells (Figure 9b). Under prolonged LGFU (200-400 pulses) with such high amplitude, two cells retreated outward (Figure 9c,d). This means that a shear force on the substrate is formed by cavitational collapse, along the radial directions from the center, which pushes two cells away from each other. The result in Figure 9 also confirms that the cultured morphology in single cells can be significantly deformed by LGFU produced in the overthreshold regime for cavitation. 
(a)

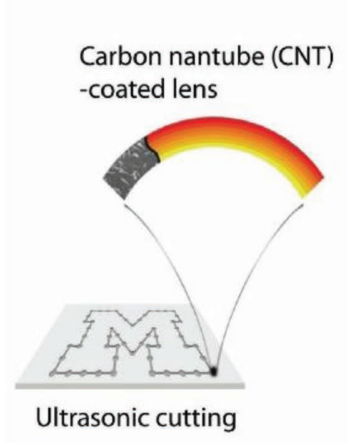

(b)

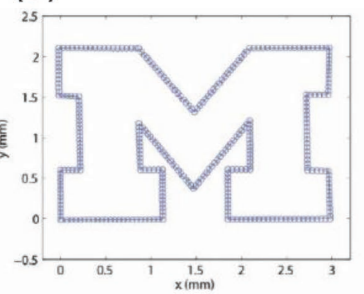

(d)

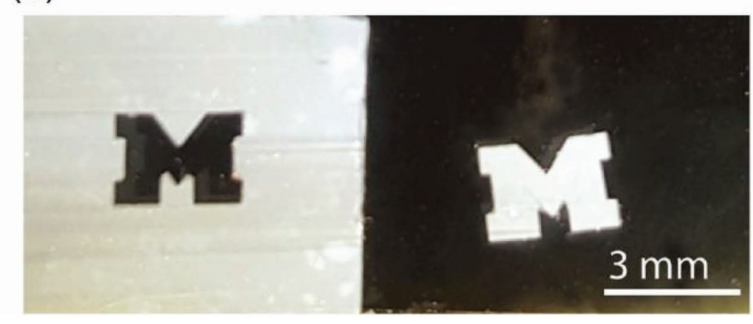

Figure 10. Invisible sonic scalpel. a) Photoacoustic cutting of tissue-mimicking gels. The laser beam and focused photoacoustic transmitter are fixed while the gel samples mounted on a 3D motorized stage are moved. b) Predefined cutting path used for sonic cutting. c) Photo of a gel immediately after sonic cutting and before removal of the sliced part (M-letter). The red arrows indicate the width of the kerf $(\approx 50 \mu \mathrm{m})$. d) Photo of the same gel with the inner part separated. Adapted with permission. ${ }^{[47]}$ Copyright 2017, Wiley-VCH.

\subsubsection{Invisible Sonic Scalpel}

Microcavitation produced by focused photoacoustic pulses was used to realize high-precision sonic scalpel, as illustrated in Figure 10a. ${ }^{[47]}$ Although the CNT lens in ref. [12] was capable of creating cavitation on the sound-reflecting interfaces such as a glass surface or air/liquid interface, it could not produce cavitation in the free field with no reflecting surfaces. This is because free-field cavitation in water (no pressure enhancement by pressure interference) requires much higher-pressure amplitudes than cavitation occurring on sound-reflecting interfaces, and specifically negative pressure amplitudes for cavitation need to be $>30 \mathrm{MPa}$. To achieve such high-pressure amplitudes, we optimized the CNT photoacoustic transmitter in a separate work ${ }^{[42]}$ such that the optimized CNT transmitter had a lower f-number (0.61) for tighter focusing, compared to that reported in a previous study. ${ }^{[12]}$ The focal gain by the CNT transmitter with an f-number of 0.61 was found to be 220 . In a study, ${ }^{[42]}$ we first demonstrated free-field cavitation and characterized the detailed characteristics of the optimized CNT lens having the low f-number.

Using the capability to create free-field cavitation in a controllable manner, we demonstrated high-precision cutting of tissue-mimicking gels. Because the gels used were mostly composed of water, cavitation was created in the middle of the gels when the photoacoustic pulses were applied. We found a cloud of microbubbles were generated within the focal volume and were eventually merged into single large bubbles. It was observed that oscillating microbubbles induced considerable mechanical effects to the surrounding gel, thus fractionizing the gel. The interesting application was introduced by utilizing such mechanical effects. We were able to cut the gel along a programmed path (Figure 10b), which was done by moving the gel sample through a motorized stage with respect to the position of the microbubble. The kerf of the sonic cutting was determined by the size of the microbubbles and was found to be sufficiently small (see the kerf in Figure 10c), allowing highprecision cutting (Figure 10d).

We showed that the sonic cutting is a nonthermal process, as temperature increase was estimated to be negligible. Therefore, this approach could be effective for targeted, noninvasive surgery. Such targeted approach can minimize collateral damages to surrounding tissues. By incorporating high-resolution imaging modalities, one can delineate margins of lesions and accurately remove them by using the sonic cutting. Also, we believe that this high-precision sonic cutting could be very effective in removing small lesions close to critical nerves. Moreover, we anticipate this sonic cutting can be even applied to targeted surgery of brain, requiring high precision.

\subsubsection{Nozzle-Free Liquid Jetting}

Photoacoustic pulses generated by a CNT photoacoustic transmitter was implemented to demonstrate nozzle-free, high-speed liquid microjetting, as illustrated in Figure 11a. ${ }^{[48]}$ Such nozzlefree jetting may address issues associated with conventional jetting approaches based on nozzles, which are prone to nozzle clogging. This nozzle clogging could be a problem, particularly when jetting liquids are comprised of materials such as flakes, particles, or surface adhesive biological molecules. The nozzle-free microjetting was realized by using high-amplitude photoacoustic pulses, which were sufficiently strong to create microcavitation at the subsurface of the air/water interface, as seen in Figure 11b. The microcavitation near the free surface pushes the adjacent water to emerge in the form of jets. In fact, when the photoacoustic pulses were focused onto the air/water interface, the photoacoustic pulses were reflected from the interface, experiencing considerable interference and thus producing high-amplitude pressure pulses. Due to this interference, the 
(a)

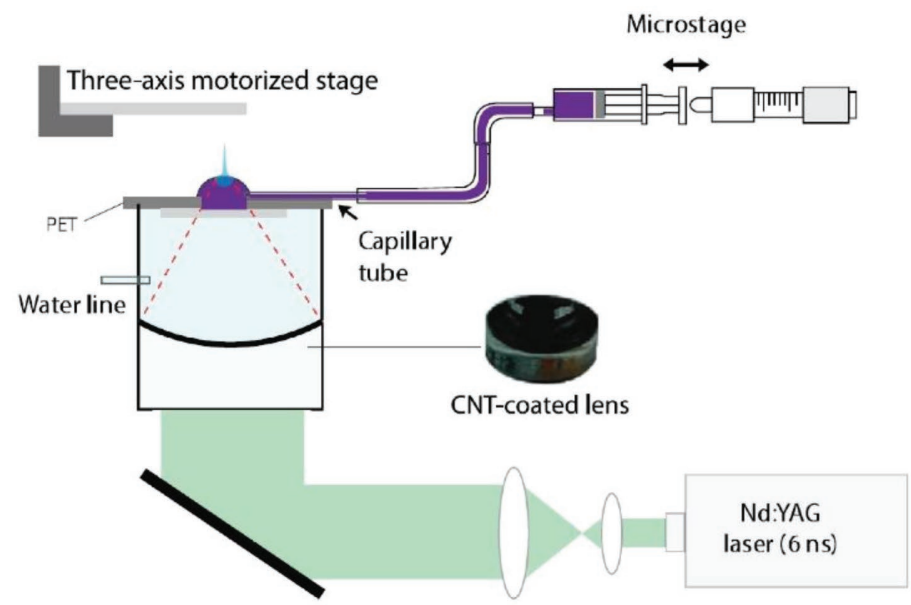

(b)

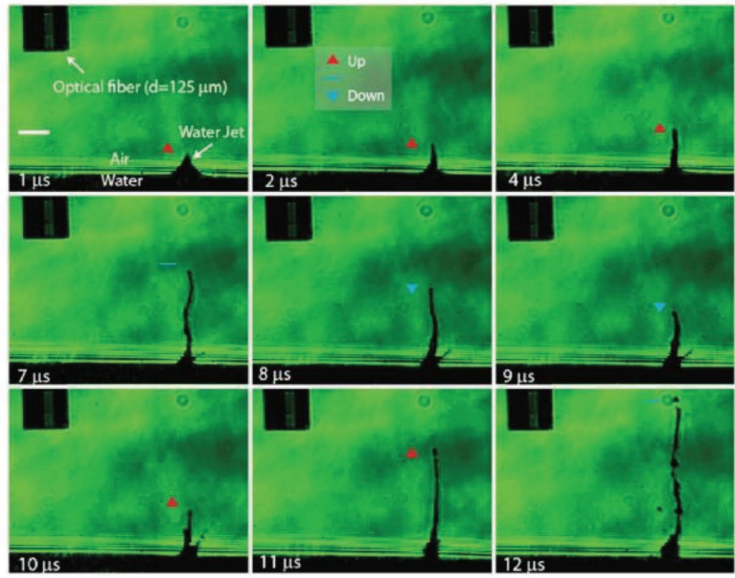

Figure 11. Nozzle-free liquid microjetting by a CNT photoacoustic transmitter. a) Experimental setup of liquid microjetting. Focused photoacoustic pulses generated by a CNT-coated concave transmitter are focused on the air/water interface. Liquid for jetting is separated from water used for sound propagation through an acoustically transparent thin membrane. The location of the interface with respect to the focal point is precisely adjusted with a syringe pump attached on a microstage. b) Shadowgraph images of liquid microjets emerged from the air/water interface. The snapshots were taken at delays ranging from 1 to $12 \mu \mathrm{s}$ after the arrival of photoacoustic pulses onto the air/water interface. The sizes of produced microjets are compared with an optical fiber (diameter of $125 \mu \mathrm{m}$ ). Adapted with permission. ${ }^{[4]}$ Copyright 2015, American Physical Society.

pressure amplitude was found to be significantly increased, exceeding the cavitation threshold (negative pressure amplitude $>30 \mathrm{MPa}$ ). Also, it was observed that the short pulse width of the photoacoustic pulses was critical to the jetting performance, because the pulse width determines the location of the highpressure zone (pressure interference) as well as the position of cavitation, critically influencing the jetting behavior.

The microjets produced by high-amplitude photoacoustic pulses have shown unique features: high speed jets with microscale. We showed two interesting applications such as printing and drug injection. For the printing application, liquid ink was used and microcavitation produced by photoacoustic pulses cause the ink to be ejected onto a glass substrate. Since the column of the microjets was thin and droplets pinched off from the jets were microscale, the printing resolution was found to be $30 \mu \mathrm{m}$. Moreover, the speed of the microjets generated by the photoacoustic pulses reached approximately to hundreds of $\mathrm{m} \mathrm{s}^{-1}$. We showed that such high-speed liquid microjets effectively penetrated tissue-mimicking gels, allowing potential applications, e.g., needle-free drug injection.

\subsubsection{Spatiotemporal Drug Delivery}

Photoacoustic waves can be generated by either pulsed optical excitation or extended light modulation. Whereas photoacoustic waves produced by extended modulation were reportedly effective in improved imaging resolution, ${ }^{[49]}$ pulsed excitation is preferred for LGFU, capable of localizing mechanical effects without depositing unwanted thermal energy owing to significantly low duty cycle $(<0.001 \%)$. Such capability of LGFU could be desirable for on-demand delivery of therapeutic agents. Di et al. presented drug delivery using LGFU. ${ }^{[43]}$ They used a CBPDMS composite for photoacoustic generation (Figure 12a,b).
Also, they exhibited that LGFU is promising by triggering drug release from drug-loaded nanoparticles. They observed that the therapeutic agents released by LGFU were proportional to pressure amplitude as well as treatment time. Notably, the amount of released drug was varied depending on a location with respect to the focus. They explained that such an effect resulted from LGFU-induced cavitation. To verify this, they conducted a control experiment where degassed PBS solution was used to reduce cavitation probability. Indeed, they found that the degassed solution showed a significant decrease in the released drug concentration. Moreover, the LGFU treatment exhibited the antiproliferation capability of the released drug triggered by LGFU, showing that the HeLa spheroid of the smallest size at day 7 (Figure 12c).

\subsubsection{Photomechanical Cell Detachment}

Retrieving sister cells separately inside enclosed microfluidics remains challenging, but it is highly desirable for further analyses of critical cells. Chen et al. demonstrated a selective photomechanical detachment of single cells from enclosed microfluidics. ${ }^{[50]}$ As shown in Figure 13a, effective cell detachment was enabled by using mechanical force induced by pulsed laser excitation of a carbon nanotube-PDMS composite (Figure 13c,d). Single cells cultured on the CNT surfaces are selectively detached in the microfluidic chamber (Figure 13b). The irradiated optical energy is converted through the CNT photoacoustic film to mechanical energy, possibly emitted in the form of photoacoustic pulses. Although the propagation of the photoacoustic pulses is not a main contribution to the cell detachment, the single cell detachment can be explained with the efficient photoacoustic (light-to-mechanical) conversion in the CNT composite film and corresponding 
(a)

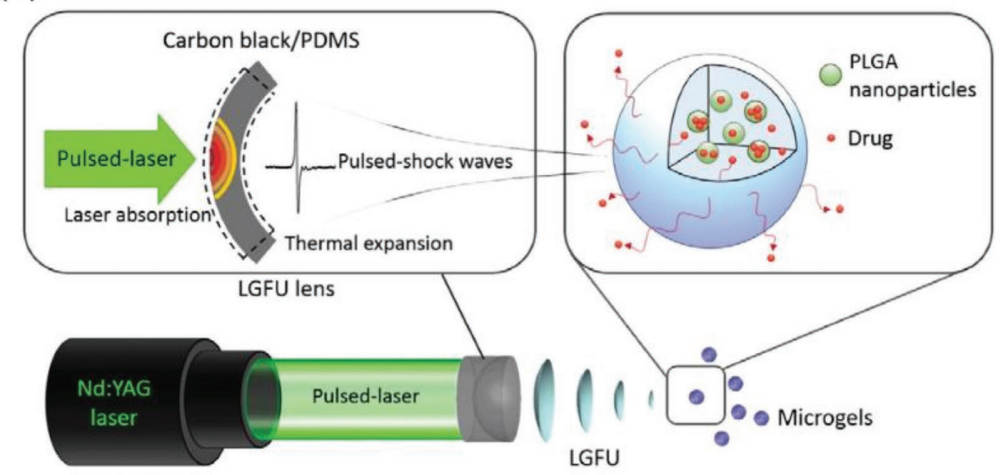

(b)

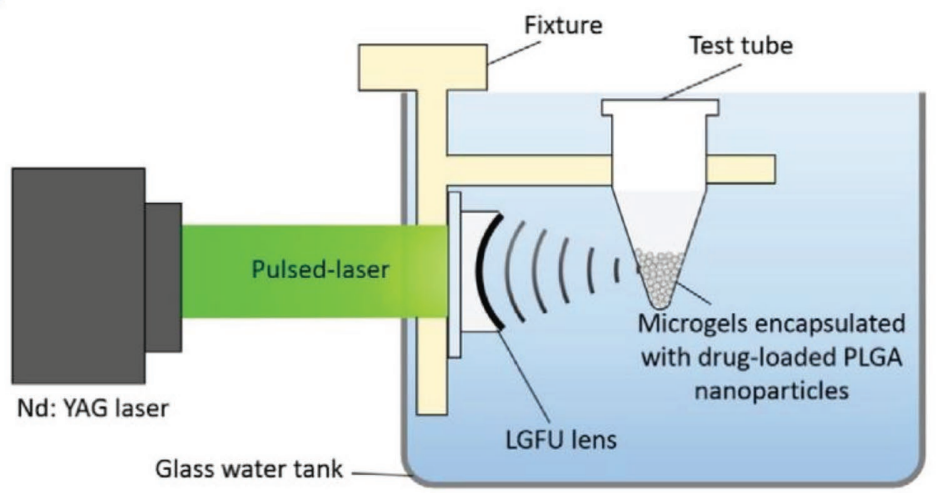

(c)

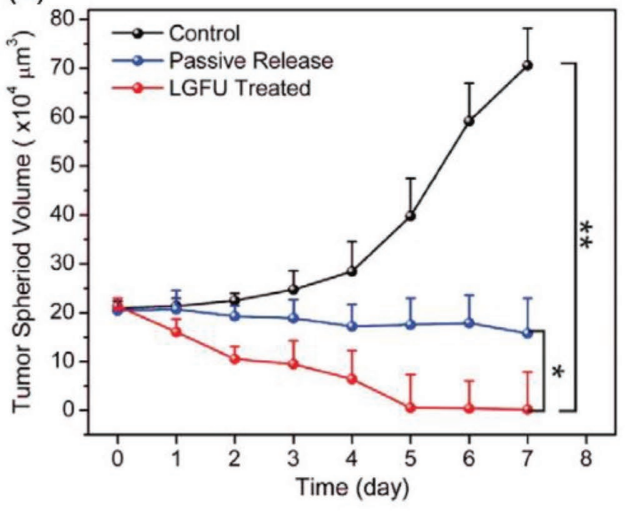

(d)

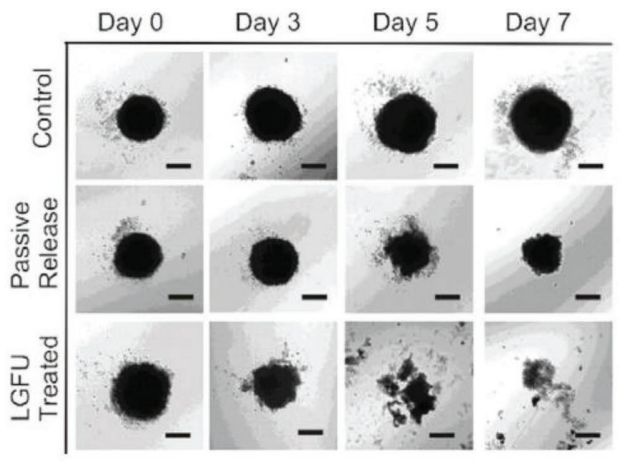

Figure 12. Spatiotemporal drug delivery using a photoacoustic transmitter. a) Schematic of photoacoustic pulse-induced drug delivery system. Focused photoacoustic pulses cause cavitation and oscillation of microgels containing drug-loaded PLGA nanoparticles, promoting drug release from microgels. b) Experimental setup. c) Normalized sizes of HeLa tumor spheroid. d) Tumor morphologies over a course of days (0, 3, 5, and 7) after the treatment. The scale bars correspond to a length of $100 \mu \mathrm{m}$. Adapted with permission. ${ }^{[43]}$ Copyright 2015 , Elsevier.

mechanical effect (shear force). They showed that single cells were selectively detached and removed from the original location (Figure $13 \mathrm{e}-\mathrm{j}$ ). They confirmed that the detached cell was viable, and its membrane was preserved without biochemical modification.

\subsection{High-Resolution Imaging, Sensing, and Detection}

Conventional ultrasound probes (e.g., piezoelectric transducers) may suffer from fabrication issues (e.g., dicing of active elements and electrical connection of the elements). These issues get worse when the ultrasound frequencies are increased. To address these issues, all-optical ultrasound is alternatively used as a powerful tool. Moreover, all-optical ultrasound is desirable in realizing minimally invasive medical imaging. By defining active source elements with optical beam size, optical methods are flexible and can allow for real-time 2D or 3D high-resolution imaging. In addition, ultrasound can be produced in a light-absorbing coating on an optical fiber tip. When combined with fiber optic ultrasound detectors, fiber optic ultrasound generator constitutes all-optical ultrasound probes for pulseecho ultrasound imaging.

Photoacoustic pulses generated by pulsed lasers have the characteristics of wide bandwidth in a wide range of frequencies (from the hundreds of $\mathrm{kHz}$ to tens of $\mathrm{MHz}$ ). The sensitive detection of broadband photoacoustic pulses is desirable in many applications ranging from photoacoustic tomography, and microscopy, to clinical ultrasound imaging. When the sensitivity is not sufficient, waveforms and reconstructed images can be incorrect. The insufficient sensitivity may be one of the limitations presented by piezoelectric ultrasound receivers. The performance limitations include a highly directional response at $\mathrm{MHz}$ frequencies that is caused by increasing acoustic sensitivities with piezoelectric element sizes on a millimeter to centimeter scale. By addressing these limitations, optical ultrasound sensing is considered a viable alternative.

\subsubsection{All Optical Ultrasound Probe}

By implementing such optical methods, Hou et al. showed 3D ultrasound imaging, which was based on an integrated photoacoustic transducer. They combined an FP cavity for detection and photoacoustic transmitters using absorbers such as gold nanostructure ${ }^{[35]}$ and carbon black PDMS. ${ }^{[13]}$ The optical absorbers were surrounded by an FP cavity, enabling pulse-echo mode operation with high frequencies and large bandwidths. The photoacoustic signals were produced by laser 


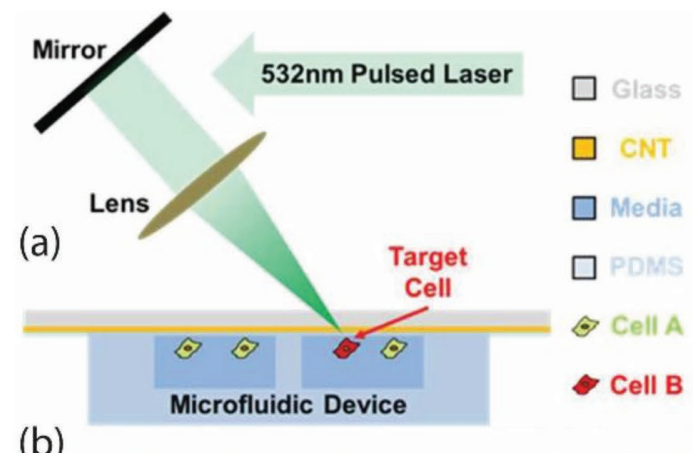

(b)
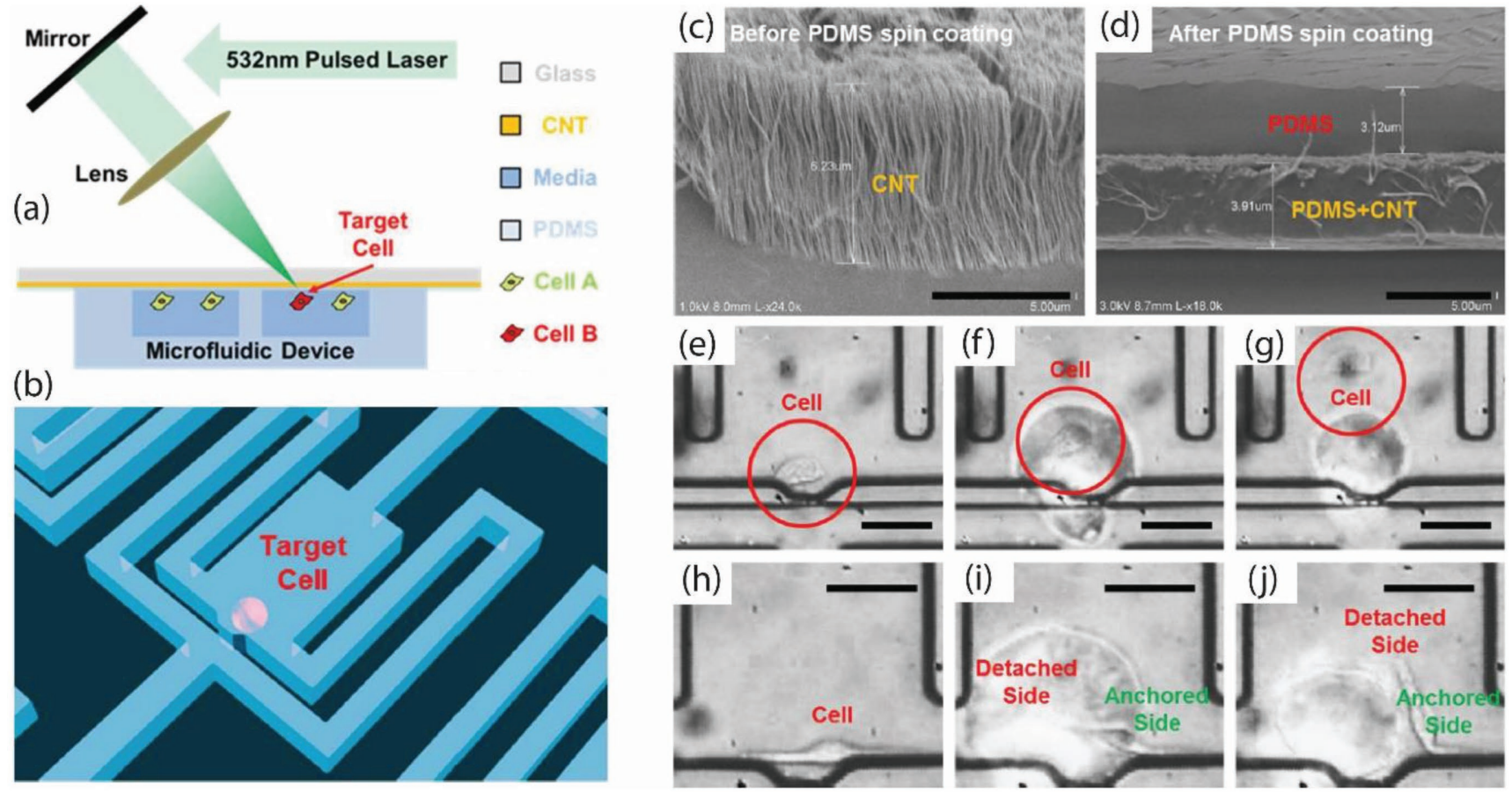

Figure 13. Single-cell detachment. a) Single-cell detachment setup with b) the microfluidic chamber. c,d) SEM images of the CNTs before and after PDMS coating (scale bar: $5 \mu \mathrm{m}$ ). Laser detachment of a cell: e) intact cell, f) immediately after laser illumination, and g) cell detachment (scale bar: $50 \mu \mathrm{m}) . \mathrm{h}-\mathrm{j})$ Partial detachment of a second type of cell (scale bar: $50 \mu \mathrm{m}$ ). Adapted with permission. ${ }^{[50]}$ Copyright 2017, American Chemical Society.

irradiation onto the carbon black PDMS film. ${ }^{[14]}$ The FP cavity relies on an light reflection change induced by an acoustic wave, permitting sensitive detection. By employing a specific arrangement of transmit and detection elements, small targets of $50 \mu \mathrm{m}$ diameter were successfully imaged. The geometry of the imaging targets was reconstructed by using conventional beamforming algorithms. This proved the full potential of this approach for 3D ultrasound imaging.

\subsubsection{Optical Fiber-Based Ultrasound Probe}

By integrating with optical fibers, such ultrasound probe based on optical approaches can be effectively miniaturized. Colchester et al. demonstrated optical fiber-based ultrasound probe. ${ }^{[51]}$ The ultrasound probe, made of two optical fibers, used one for generating ultrasound and the other for detecting ultrasound (Figure 14a). Photoacoustic pulses were produced by a fiber optic (a)

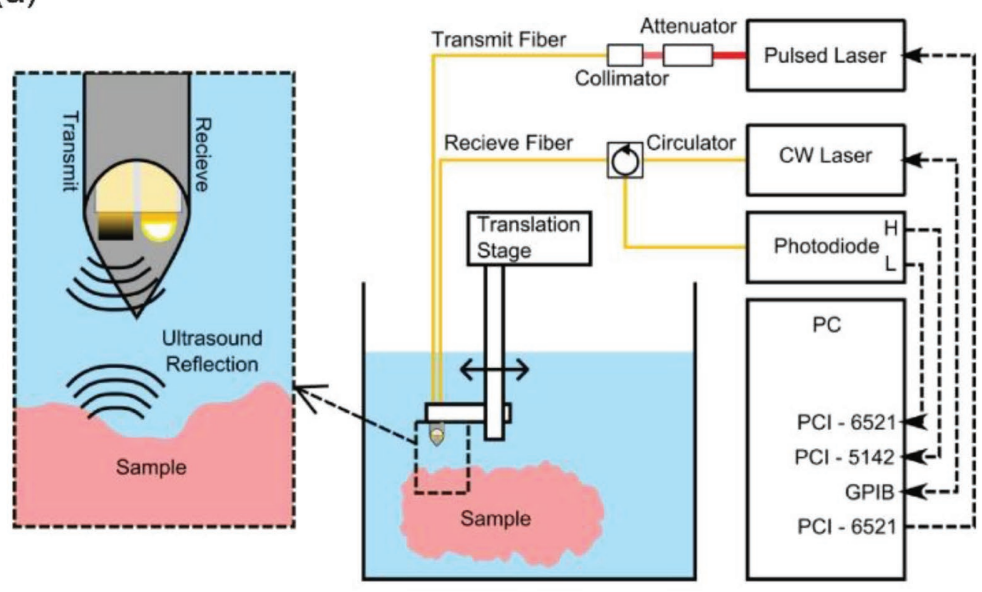

(b)

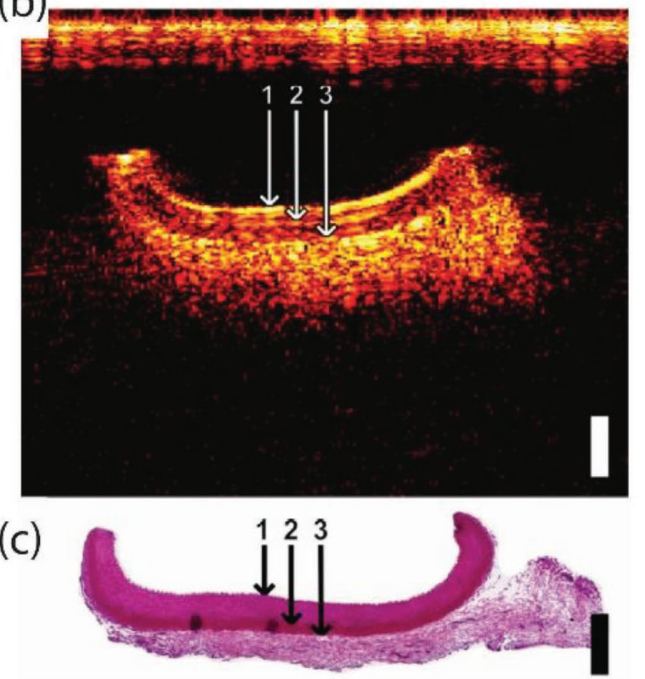

Figure 14. Broadband miniature photoacoustic probe. a) All-optical imaging system setup with the ultrasound generation and detection fibers. b) Optical ultrasound image of the carotid artery. c) Corresponding histological cross-section. The number labels help the comparison between (b) and (c). Adapted with permission. ${ }^{[51]}$ Copyright 2015, OSA. 
(a1)
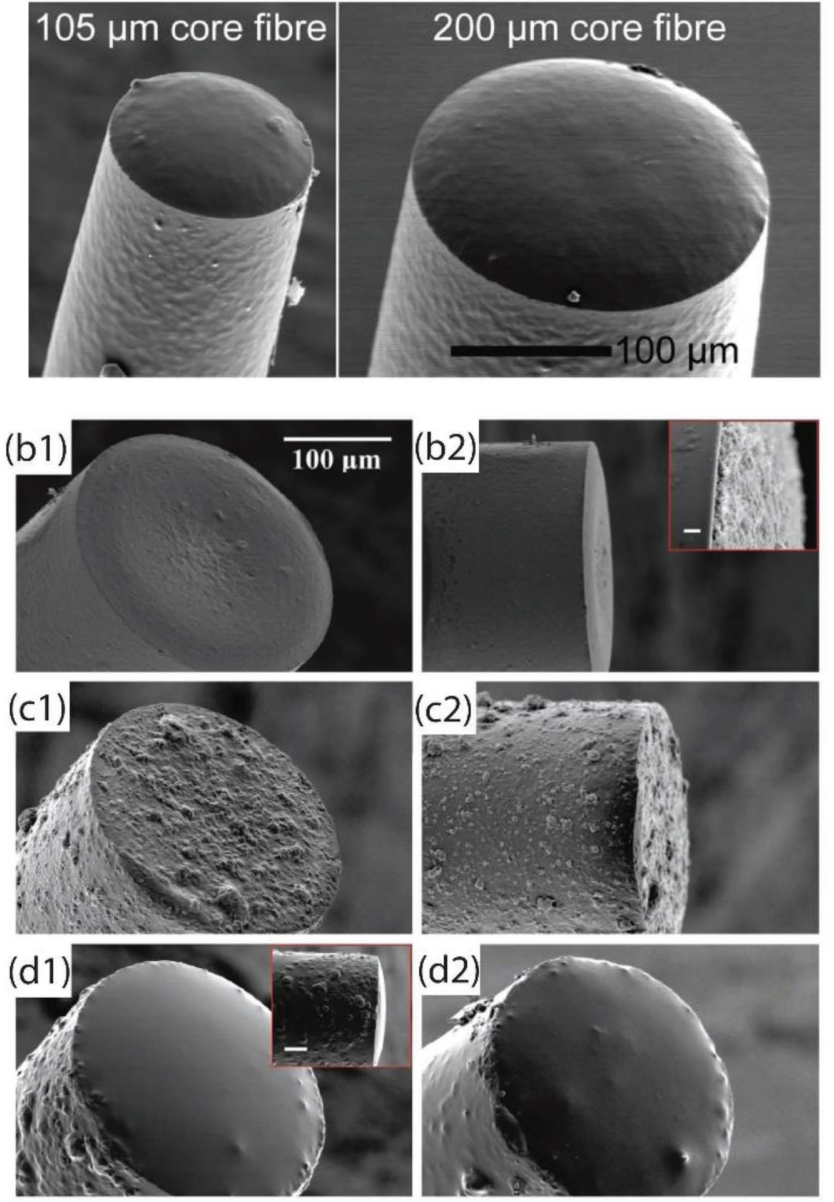

Figure 15. Fiber optic photoacoustic transmitter. a) SEM images of the optical fibers coated with the CNT-PDMS composite. Adapted under a Creative Commons Attribution 3.0 Unported License. ${ }^{[10]}$ Copyright 2014, The Authors. b) MWCNT-xylene coating (b2, side-view). c) MWCNTgel coating (c2, side-view). d1) MWCNT-gel/PDMS coating and d2) MWCNT-PDMS integrated coating. Adapted with permission. [11] Copyright 2016, The Authors.

photoacoustic transmitter (200 $\mu \mathrm{m}$ core silica fiber) (see Figure 15 for example), ${ }^{[10]}$ while the optical fiber for detection used an FP optical cavity. This ultrasound probe was precisely moved to produce virtual transmit/receive elements, permitting pulse-echo ultrasound imaging with high imaging resolution. The reconstructed images showed the high spatial resolution at a depth of $3.5 \mathrm{~mm}$. They showed that ultrasound imaging of vascular tissues from animal models matched with the histological images (Figure 14b).

Other recent papers related to high-resolution imaging can be found elsewhere. ${ }^{[52,53]}$ Moreover, there is a significant progress in high-resolution imaging based on photoacoustic transmitters. Two recent review articles cover high-resolution imaging. ${ }^{54,55]}$

\subsubsection{Planar Etalon for Ultrasound Detection}

To offer a practical alternative to conventional photoacoustic systems (piezoelectric-based), Zhang et al. demonstrated an optical sensing of photoacoustic waves, enabled with a Fabry-Perot polymer film sensor, whose active element size can be optically defined and significantly smaller than can be attainable with piezoelectric receivers (Figure 16a). ${ }^{[56]}$ They exhibited 3D imaging of soft tissues to desired depths with high resolution. In this system, element dimensions, optically defined, were a few tens of micrometers, providing adequate detection sensitivity (noise equivalent pressure of $0.21 \mathrm{kPa}$ ).

The FPI scanner, consisting of a polymer (Parylene) spacer sandwiched by a pair of optically reflective mirrors, can detect the optical thickness variation of the spacer that is acoustically induced (Figure 16b). They implemented a second galvanometer for 2D mapping of the sensor output. Also, for photoacoustic imaging, they used a new sensor design capable of operating in backward mode by providing the transparency of the FPI mirrors (near infrared). They presented high-resolution 3D imaging of absorbing objects. Although targets of interest were immersed in an light-scattering solution, the probe enabled accurate imaging of the targets (a dye-filled knotted tube) (Figure 16c).

\subsubsection{Microring Resonator for Ultrasound Detection}

It is known that for high-resolution medical imaging, including conventional ultrasound imaging and photoacoustic imaging, we need to use $>20 \mathrm{MHz}$ ultrasound to ensure decent analysis and correct diagnosis. It requires both small size and broadband for the detectors to avoid spatial averaging effect from these high-frequency ultrasound pulses and to provide a good axial resolution. Conventional ultrasound detectors such as PVDFbased needle hydrophones cannot fulfill broadband, small size and sensitivity at one same time. Optical detection approach, where optical output power is modulated by the ultrasound, can provide broadband response, sensitivity, small detecting area as well as wide-directivity, among which, polymer microring developed by T. Ling et al. stands out with its extreme sensitivity and acoustic response bandwidth. ${ }^{[57]}$

The microring resonator is a nanoimprinted polystyrenebased extruding ring structure, as shown in Figure 17a. Nanoimprinting lithography ensures the high throughput and reproducibility of these miniature polymer structures. $780 \mathrm{~nm}$ light is BUT-coupled from a single mode fiber into a straight waveguide and outputted in a multimode fiber connected with the photodetector. At the resonance wavelength, most of the input optical energy will get lost when the light is looping in the ring area and the transmission spectrum of the resonator will show resonance dips, where the input probing light is tuned and fixed at. An incoming acoustic wave onto the polymer, due to the optoelastic effect and deformation of the resonator, will modulate the output light power which depicts the acoustic signal, as shown in Figure 17b.

This optical ring ultrasound detector achieves a high sensitivity of $\mathrm{NEP} \approx 230 \mathrm{~Pa}$, a flat acoustic response bandwidth of $1-90 \mathrm{MHz}$ at $-3 \mathrm{~dB}$ and wide directivity of $40^{\circ}$ at $-6 \mathrm{~dB}$. These records have been updated to an NEP of $105 \mathrm{~Pa}, 1-350 \mathrm{MHz}$ at $-3 \mathrm{~dB}$ with the following-up works. ${ }^{[58]}$ Moreover, with this $\approx 300 \mathrm{MHz}$ bandwidth ultrasound sensor, we can get a much better axial resolution in photoacoustic imaging. Also, we can analysis the higher frequency component from the PA signals of cellular-size subjects, 
(a)

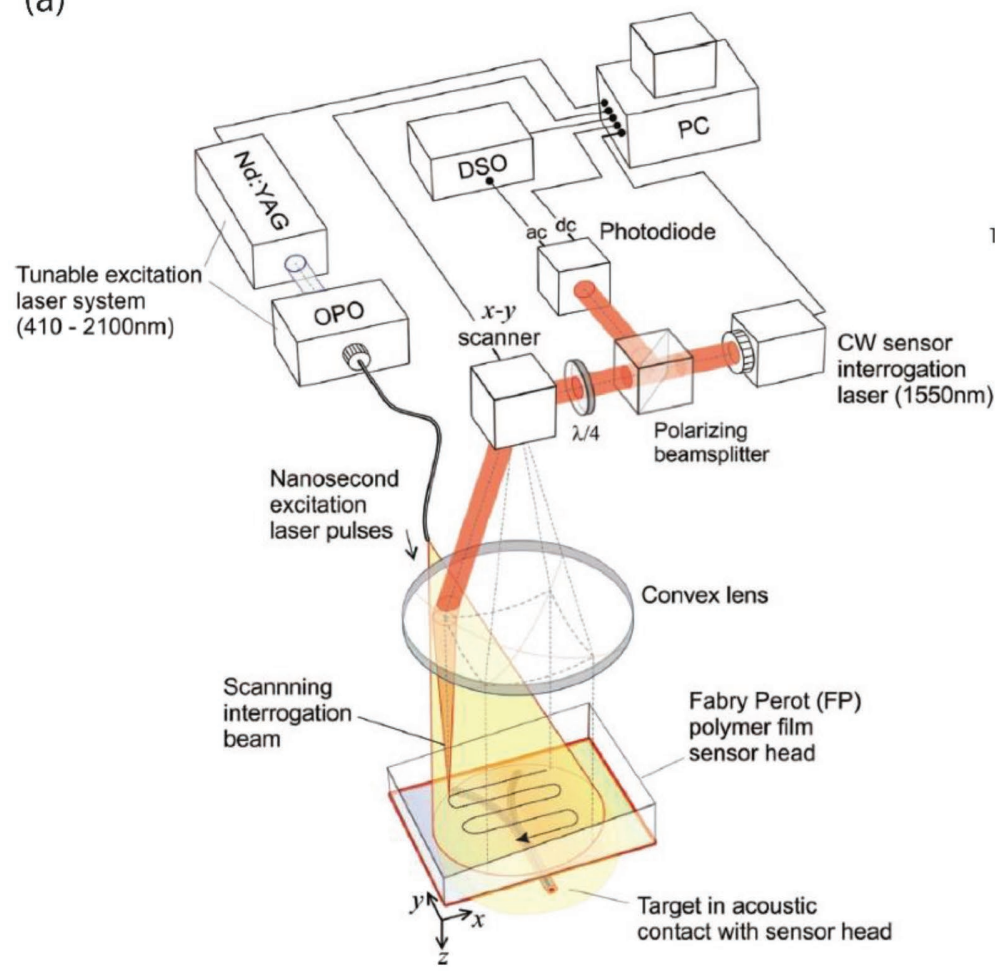

(b)

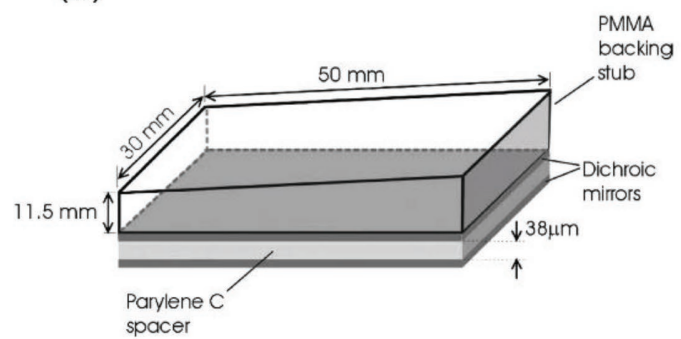

(c)
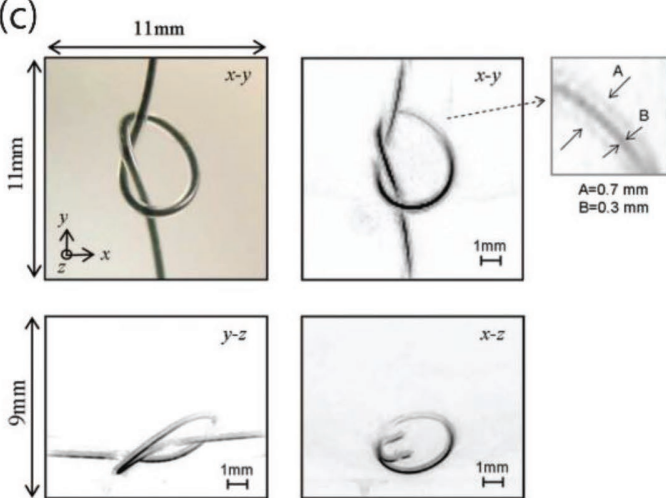

Figure 16. a) Photoacoustic scanner consisting of the Fabry-Perot (FP) sensor head. b) Schematic of FP sensor head made of a polymer film spacer between two mirrors. c) Photoacoustic images of dye-filled knotted tube. Top left: photograph of tube. Top right: $x-y$ of 3D photoacoustic image. Lower left: $y-z$, lower right: $x-z$, far right: close-up of $x-y$. Adapted with permission. ${ }^{[56]}$ Copyright 2008, OSA.

and we can relate the spectrum curve regression parameter with the morphology of the subjects. ${ }^{[58]}$ Finally, this silicon-based fabrication makes $1 \mathrm{D}$ and $2 \mathrm{D}$ arrays possible, and facilitates the high-resolution photoacoustic tomography. MRRs fabricated on optically opaque silicon substrate presented a limitation that a photoacoustic microscopy system based on this opaque substrate is only implemented in a transmission configuration. To overcome this limitation, Li et al. proposed an optical microring resonator built on a transparent substrate. ${ }^{[59]}$

\subsubsection{Real-Time Terahertz Detection}

It is well known that terahertz electromagnetic waves are very useful for imaging and charactering material properties. Thus, significant efforts have been devoted to finding detection techniques for terahertz radiation. However, existing $\mathrm{THz}$ techniques have issues, e.g., bulky. To address this issue, A new detection method is required. In an effort to find an alternative, we demonstrated efficient detection of terahertz (THz) pulses using the photoacoustic effect. ${ }^{[60]}$ We used the CNT composite of $\approx 30 \mu \mathrm{m}$ thickness, effectively absorbing $\mathrm{THz}$ radiation. By fulfilling the stress confinement condition, transient heating induced by the $\mathrm{THz}$ radiation generated high-amplitude ultrasound, which was sensed by an optical microring resonator. Thus, PA detection of terahertz pulse radiation (PADTH) method allows to reject continuous radiation, because the detector responds only to the pulsed excitation.
Generated by $\mathrm{THz}$ pulses, PA signals were detected by the PADTH detector (a noise-equivalent energy of $\approx 220 \mathrm{pJ}$ ) (Figure 18). Also, we found that the response time of the detector was short, presenting the usefulness of $\mathrm{THz}$ detection. To demonstrate practical applications, THz imaging of patterned metal foil was conducted with the PADTH detector (Figure 19). We showed that the PADTH detector performed better than a commercial pyroelectric detector. In summary, the PADTH enables various $\mathrm{THz}$ applications laid over a wide spectral range.

\subsubsection{Plano-Concave Microresonator}

Motivated by the advantages of optical sensors such as FabryPerot interferometers, microrings, and in-fiber Bragg gratings, Guggenheim et al. demonstrated a plano-concave microresonator, an optical ultrasound sensor that achieves high $Q$-factors of $\left.>10^{5}\right)$. Such high $Q$ sensor provides very high sensitivity. ${ }^{[61]}$ The sensor, using a focused laser beam, consists of two highly reflective mirrors encompassing a plano-concave microcavity, as shown in Figure 20a. They fabricated the concave cavity by depositing a droplet of liquid polymer onto a substrate. Then, the liquid droplet was UV-cured for a solid cavity. The device relies on the same principle as the flat FPIs. In principle, optical power is modulated by an incident acoustic wave and a consequent change in the cavity optical thickness, and is detected by a photodetector. Unlike flat surfaces, the concave surface provides a strong optical confinement by matching its curvature with that of the diverging 
(a)

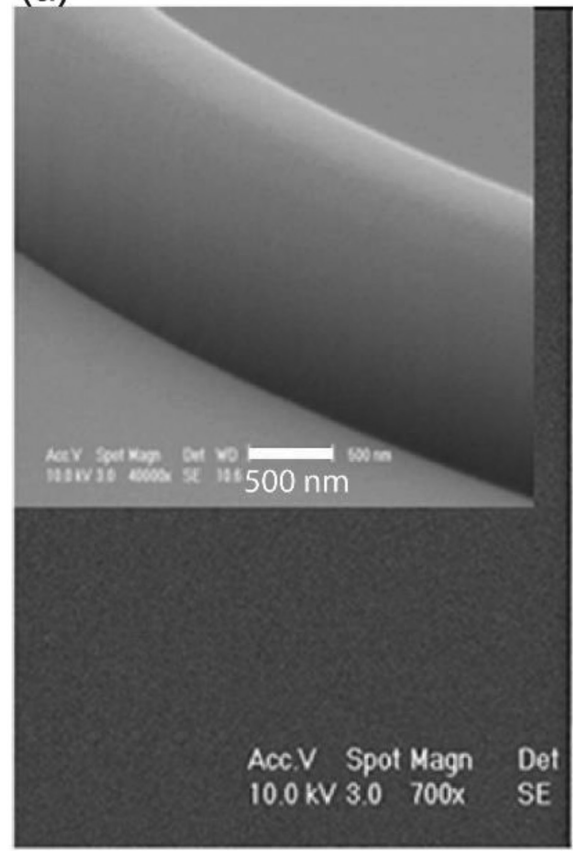

(b)

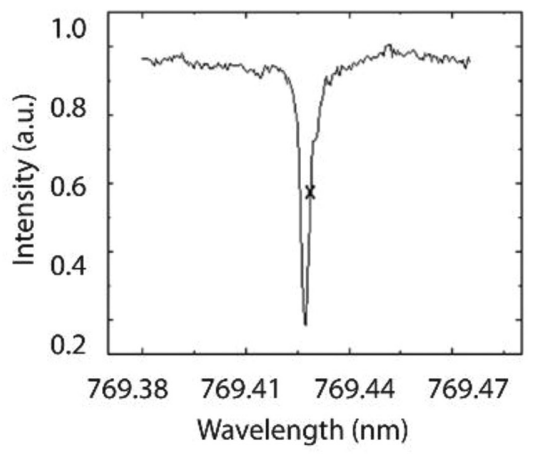

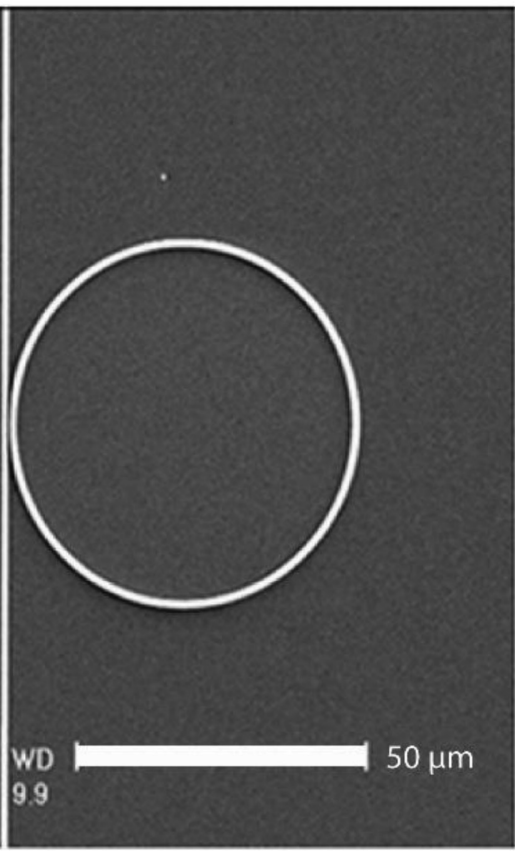

(c)

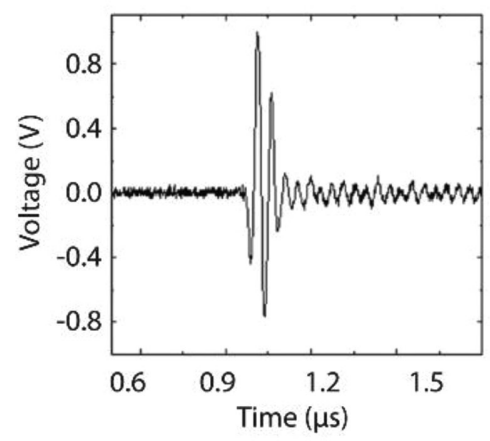

Figure 17. Polymer microring resonator. a) SEM image of a polymer microring with a straight waveguide (inset of the sidewall of the microring). b) Transmission spectrum of polymer microring and c) acoustic wave measured by the microring. Adapted with permission. ${ }^{[57]}$ Copyright 2011, AIP Publishing LLC.

beam. The plano-concave sensor provides an order-of magnitude higher $Q$-factor of 30000 , compared to a planar FP etalon (Figure 20c). A group of sensors with different thickness showed a trade-off between sensitivity and bandwidth, presenting a very low noise-equivalent pressure of $1.6 \mathrm{mPa}$ per $\sqrt{\mathrm{Hz}}$ with increased thickness. They presented a flat frequency response characteristic of an ideal broadband detector (Figure 20d). Moreover, they extended the free-space illuminated sensors to optical-fiber-based microresonator sensor (Figure 20b), which was beneficial in producing a smaller cavity thickness of $16 \mu \mathrm{m}$.

\subsection{Generation of Complex Photoacoustic Waveforms}

Motivated by efficient photoacoustic generation in recently developed polymer composites, many other applications, which cannot be easily realized with conventional approaches, have been reported. Particularly, recent efforts have been devoted to generating more complex wave fronts than a single focused or planar wave. These complex wave fronts are useful in a myriad of applications such as particle manipulation, nerve stimulation, and ultrasound therapy. For example, one may find it more effective to use multifoci wave fronts for maximizing therapeutic effects by simultaneous activating therapeutic agents over a wide region. Also, this approach can reduce treatment time, compared to single-focus approaches. In addition, the waveform needs to be changed in order to control nonlinear effects such as shock wave and cavitation. There may be a situation where nonthermal mechanical effects are desirable with minimal heat. In this case, one would need a specific waveform with positive amplitude lower than negative amplitude. Here, the low positive amplitude can suppress shock waves that can cause unwanted heat, while the high negative amplitude can give rise to inertial cavitation that is known as a nonthermal process. Moreover, photoacoustic waves could be applicable for mechanical stimulation of nerves (e.g., neuromodulation in stimulation of sensory nerves), as focused ultrasound generated by piezoelectric transducers has been used for this purpose. Photoacoustic approaches will be good for nerve stimulation requiring a high accuracy (e.g., small animal experiment). The mechanism of ultrasound stimulation is still elusive. Thus, to study the stimulation mechanism, one may benefit from precise control of photoacoustic waveforms. Recent advance in control of photoacoustic waveforms makes photoacoustic approach attractive in practical applications. However, studies for an application perspective are very limited.

\subsubsection{Photoacoustic-Based Shock Waves and Predesigned Cavitation Pattern by Structured Optical Fiber Tips}

Previous study has demonstrated that a refocusing toroidal tensile wave will be generated from the diffraction of photoacoustic wave that is excited from the circumference of a flat fiber tip in absorbing liquid. This negative tensile pressure wave is essential for cavitation bubbles formation. This makes histotripsy at fiber tips possible and can stimulate a novel, fiber-based tissue ablation utilizing the fiber's compact size and flexibility. However, conventional cleaved fibers can only allow the waves to focus along the fiber axis and cavitation clouds will only center on the same axis, limiting the flexibility of the ablation direction. M. Mohammadzadeh et al. solved this problem by modifying and shaping the fiber tips through laser machining, and successfully steer the wave front and so as the cavitation 
(a)

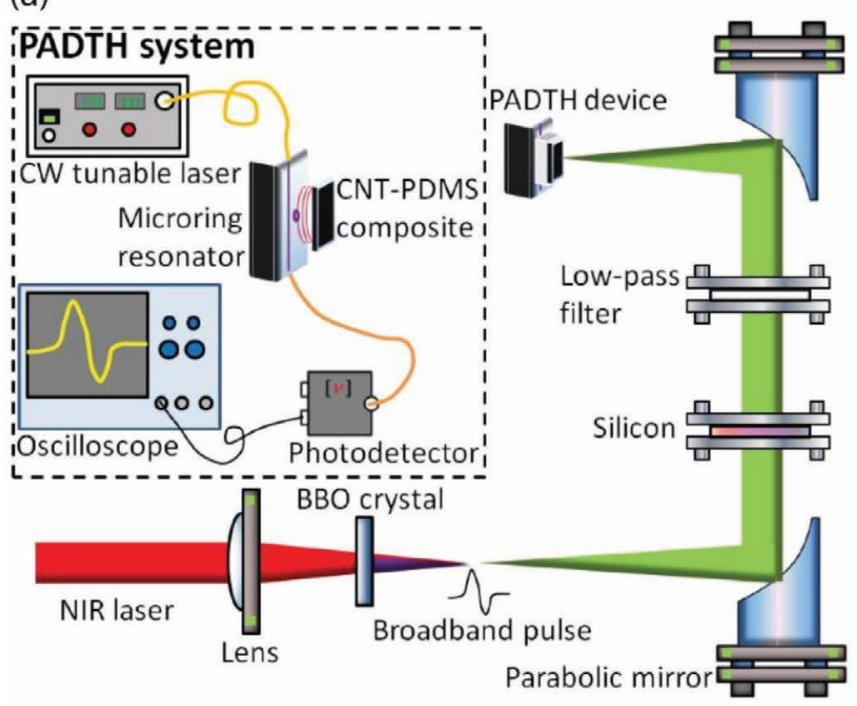

(b)

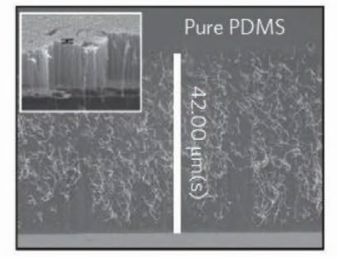

(c)

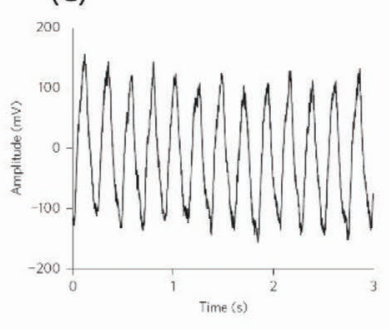

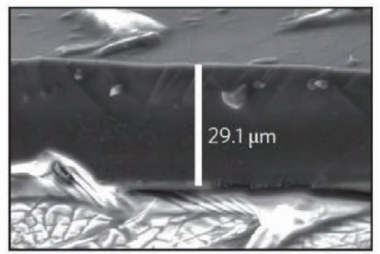

(d)

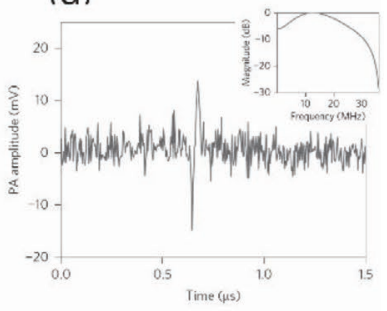

Figure 18. Terahertz radiation detection. a) Setup of THz detection. b) The structure of the CNT-PDMS composite films (SEM images). c) Terahertz radiation detected with a pyroelectric detector. d) PA waveform induced by one terahertz pulse. Inset: the spectrum of terahertz pulse-excited PA waveform. Adapted with permission. ${ }^{[60]}$ Copyright 2014, Macmillan Publishers Limited.

(a)

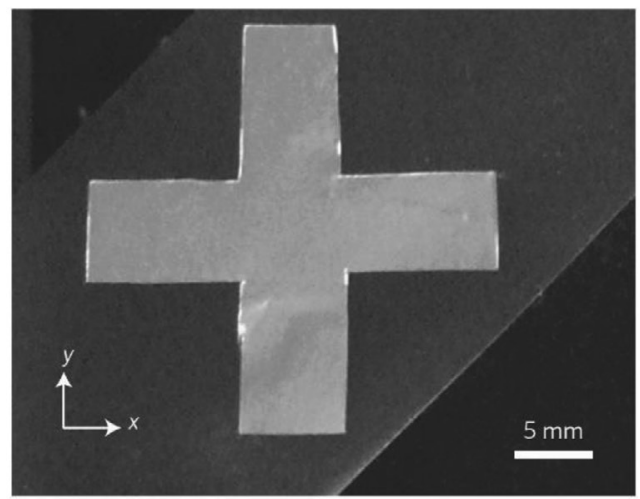

(b)

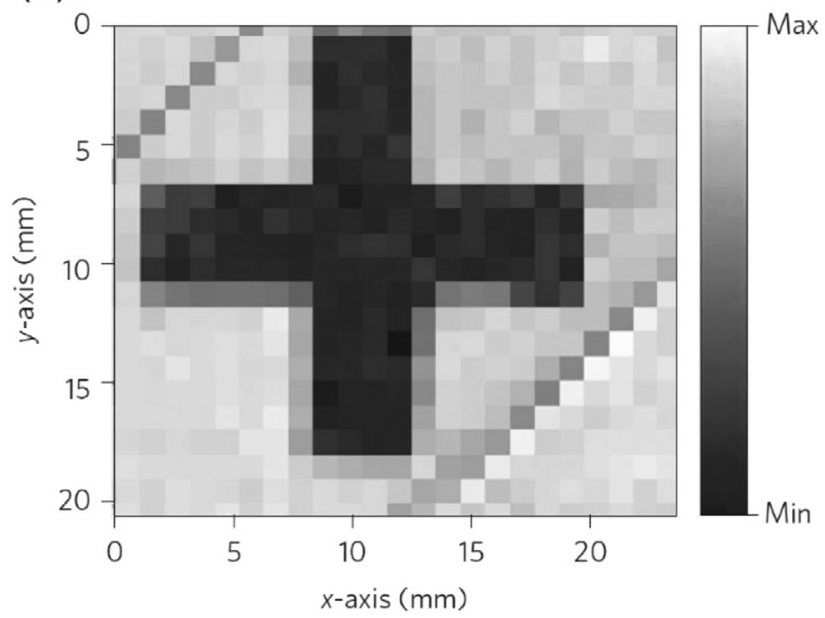

Figure 19. Photoacoustic terahertz imaging. a) Patterned aluminum foil. b) Imaging result by the PADTH detector. Adapted with permission. ${ }^{[60]}$ Copyright 2014, Macmillan Publishers Limited. clouds away from the fiber axis with an oblique fiber tip. ${ }^{[62]}$ Furthermore, they produce multiple photoacoustic waves with one single laser shot with some step fiber tip or more complicated structures.

In their work, five different tip structures have been tested. They coupled picosecond infra-red lasers to micromachined structures on the tips of cleaved multimode silica fibers as shown in Figure 21. Various wave fronts and diffracted tensile waves from all the tips were simulated using the linear wave equation model and demonstrated by experimental observation via laser shadowgraph. For the oblique cut fiber the diffracted tensile wave from tip's circumstance were steered at a predesigned angle with respect to the fiber axis, while perpendicular to the cut. Therefore, the cavitation clouds were formed in the same direction. Multiple compressive and tensile waves were realized with step, groove and ridge structure due to the separation of the illuminated area and more edges are induced. The cavitation was formed within the overlap region of the negative tensile waves and the cloud pattern could be potentially designed by considering the tensile wave propagation path. Particularly, the researchers measured the temporal pressure waveforms of the flat and step fiber tips using a fiber optic hydrophone (HFO-690, Onda, Copr. USA). At $1 \mathrm{~mJ}$ laser energy, the waveform and amplitude agree well with the linear acoustic model and prove the availability of the simulation model (Figure 22). These structured fiber tips introduce more possibilities for fiber-based histotripsy, for example, one can direct the cavitation clouds from vascular lumen to the neighboring tissue, open more flexibilities for the treatment.

\subsubsection{Arbitrary Waveforms from Photoacoustic Generation}

Conventional laser-generated ultrasound waveform is either focused or plane waves originating from a concave glass or 
(a)

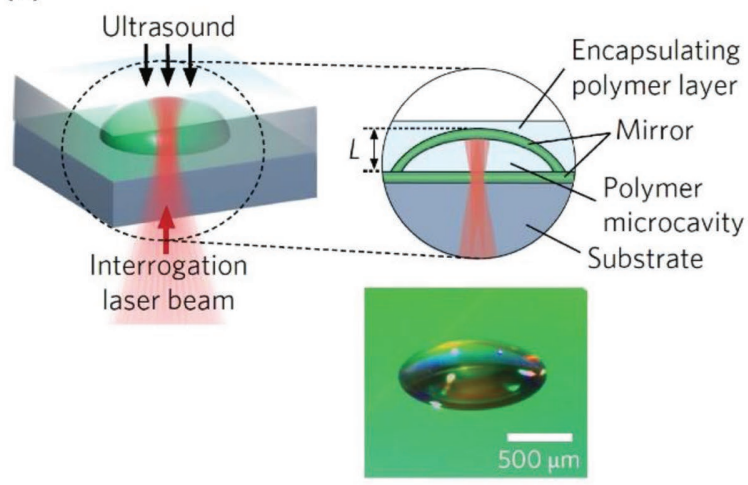

(b)

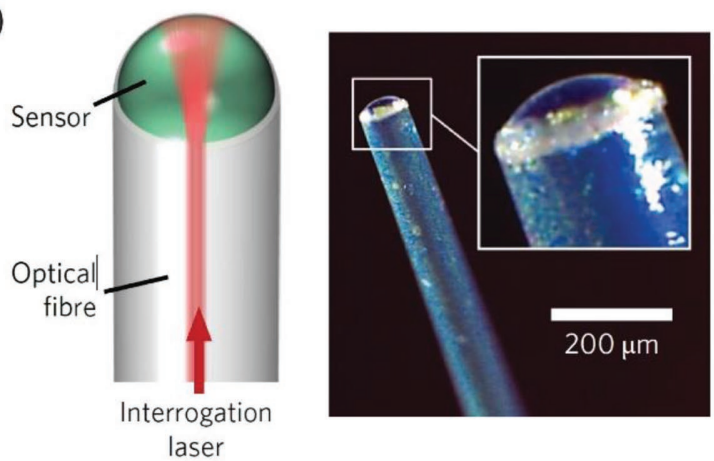

(c)

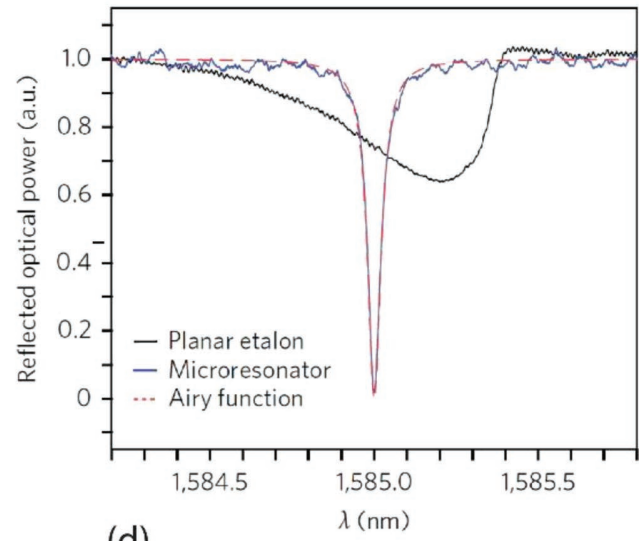

(d)

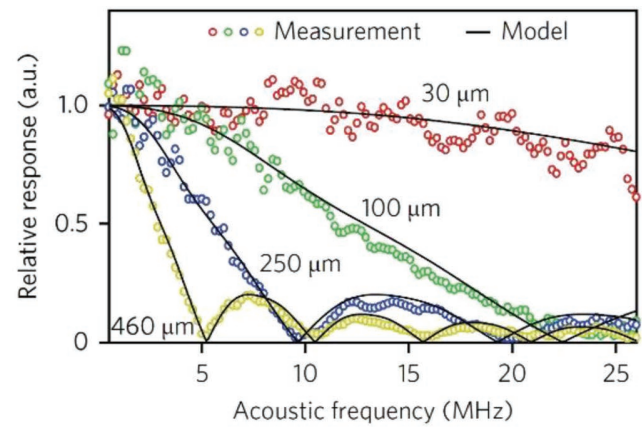

Figure 20. Optical ultrasound sensing. a) Sensor schematic of a plano-concave polymer microcavity with mirror coatings. b) Schematic of optical fiber microresonator sensor. c) Comparison of a plano-concave sensor $(Q=30000)$ with a planar etalon $(Q=3300)$. $d)$ Frequency response for different thickness. Adapted with permission. ${ }^{[61]}$ Copyright 2017, Macmillan Publishers Limited.

planar substrate. W. Chan et al. demonstrate that arbitrary waveforms in various temporal and spatial shapes can be achieved with pre-designed surface made from transparent polymers while keeping same conversion efficiency. ${ }^{[63]}$ The transparent polymers were fabricated into substrate by 3D-printing and the light absorbing layer was PDMS-CNT paint. In their work, two different shaped surfaces were fabricated, a concave surface for focusing and a stepped structure to launch two separate wave fronts. They showed that the polymer-based concave surface could generate same amplitude of focused wave as conventional glass-made lens, and a stepped surface (Figure 23) can produce double waves that are separated by $0.8 \mu \mathrm{s}$ in time domain by designing the step depth.

Three different 3D printers were tested first in making concave substrates with different loading materials for each printer. The coating on the surface was a five-layer structure: starting with MWCNT powder layer by simply brushed onto the target surface with a cotton brush, following a PDMS coating at $2000 \mathrm{rpm}$, then duplicating these two layers for once and ending with a gold layer for optical extinction. Compared with the glass
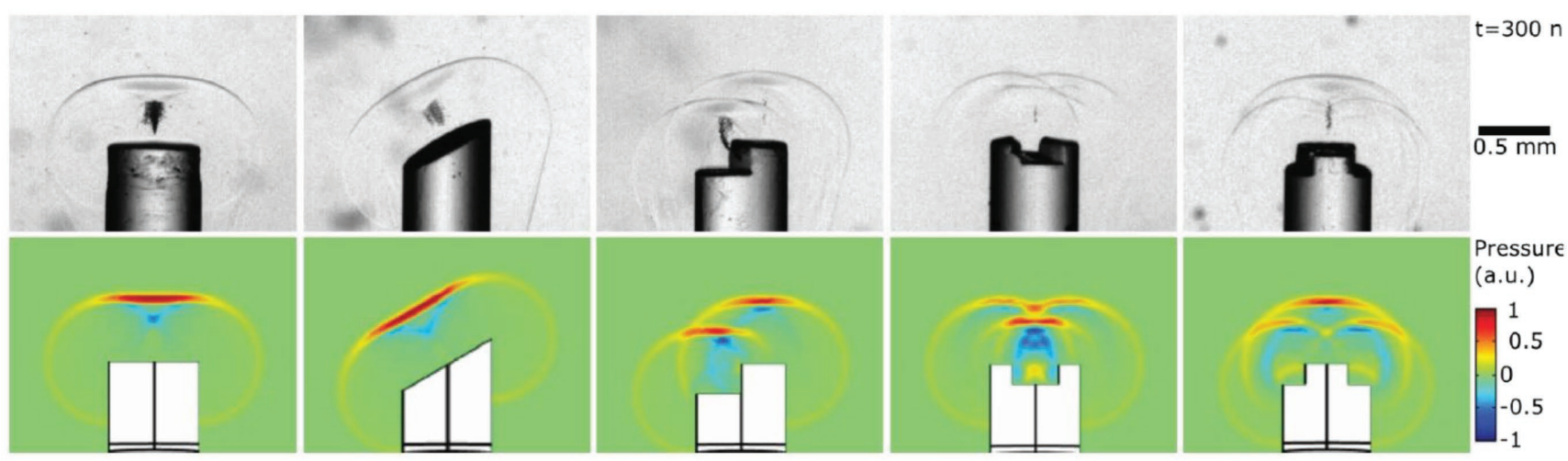

Figure 21. Acoustic transients and cavitation by structured fiber optics. Shadowgraph images (top row) and corresponding numerical solutions (bottom row). Adapted with permission. ${ }^{[62]}$ Copyright 2016, AIP Publishing LLC. 
(a) flat cleave (on-axis)

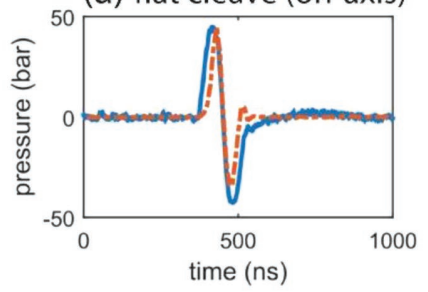

(c) flat cleave (off-axis)

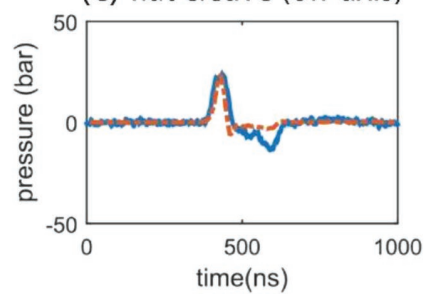

(b) Step tip (on-axis)

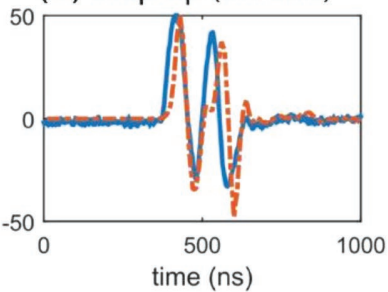

(d) Step tip (off-axis)

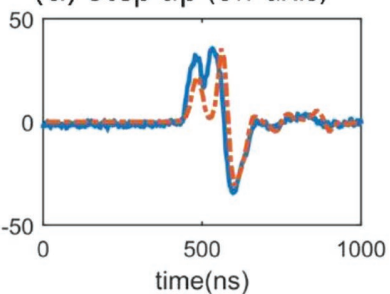

Figure 22. Pressure measurements with a fiber optic hydrophone. a) Flat cleave, on-axis $(z=600 \mathrm{~m}, y=0)$. b) Step tip, on-axis $(z=600 \mathrm{~m}$, $y=0)$. c) Flat cleave, off-axis $(z=600 \mathrm{~m}, y=300 \mathrm{~m})$. d) Step tip, off-axis $(z=600 \mathrm{~m}, y=300 \mathrm{~m})$. Adapted with permission. ${ }^{[62]}$ Copyright 2016 , AIP Publishing LLC.

concave PA lens as a gold standard, the resin concave lens made by stereolithography printer show the best conversion efficiency which was close to the glass-based one. For the stepped structure, they proved two separate waves with target temporal separation of $0.8 \mu \mathrm{s}$ are generated and the fiber hydrophone
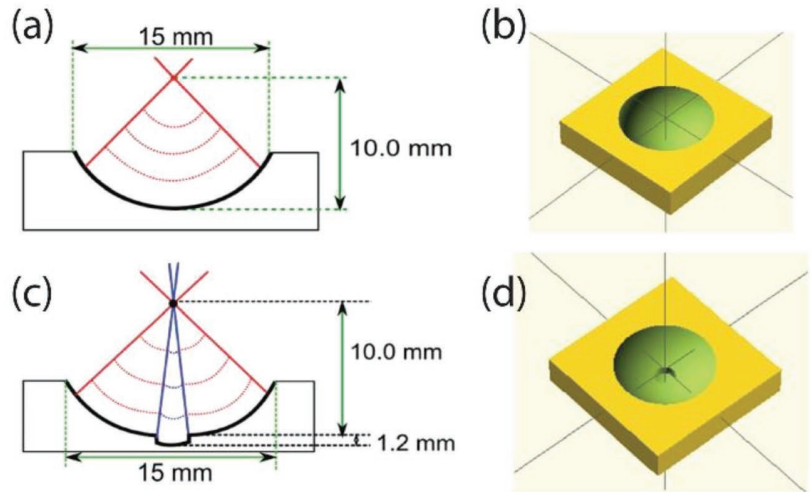

(e)

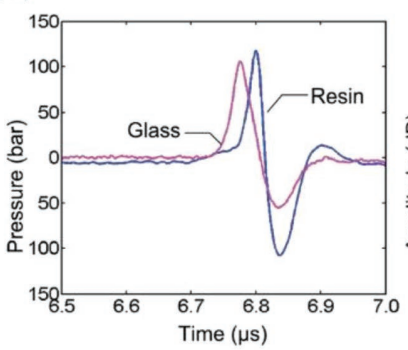

(f)

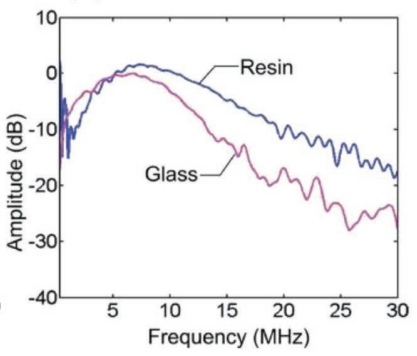

Figure 23. Generation of complex waveforms. Concave substrate for a) side view and b) 3D sketch. Stepped substrate for c) side view and d) 3D sketch. e) Measured pressure profiles at the focus. f) Corresponding frequency responses. Adapted with permission. ${ }^{[63]}$ Copyright 2016, AIP Publishing LLC. signal and shadowgraphy image prove this manipulation of wave fronts. This work offers a simple fabrication method and prove the availability of generating arbitrary waveform by designing the substrate surface, allowing future manipulation of PA waves like Bessel beams, Fresnel lens and multifoci of shock waves.

\subsubsection{Negative Quasi-Monopolar Waveform}

As ultrasound waves are composed of tensile (negative) and compressive (positive) phases, knowledge of each phase's role in ultrasound-based applications could be of great importance in understanding interaction of ultrasound with mediums used in these applications. To gain such knowledge, a method to produce monopolar pulses is critically needed. However, it is difficult to generate monopolar pulses using conventional piezoelectric transducers. Photoacoustic generation could be an effective approach, because pulsed laser excitation of lightabsorbing materials can produce short pressure pulses, whose waveforms measured near the source (near field) are dictated by the temporal profile of the laser pulse, e.g., positive photoacoustic pulses by the Gaussian temporal profile of a pulse laser. In the far field, the positive monopolar pulses are transformed into bipolar pulses due to the diffraction effect. However, generating negative monopolar pulses is believed to be challenging.

In an effort to generate high-amplitude negative monopolar photoacoustic pulses, we investigated an out-coupling effect that represents the interference of two photoacoustic pulses simultaneously generated from the same source in contact of an air-backed boundary. ${ }^{[64]}$ We found that the resulting photoacoustic pulses due to the interference had quasi-monopolar negative phases (with very small positive phases), but these pulses showed surprisingly low amplitudes compared to photoacoustic amplitudes generated with the same laser fluence in different boundaries such as a hard-backing boundary. As illustrated in Figure 24a, the low amplitudes arise because a reflected wave experiences a $\pi$ phase shift due to reflection from the air-backed boundary and destructively interferes with the other wave. To mitigate the destructive interference, a matching layer was implemented capable of controlling the interference of the two photoacoustic pulses. By properly choosing the thickness of the matching layer, photoacoustic amplitudes were observed to be significantly increased, because the reflected photoacoustic pulse has a sufficient delay, thus suppressing the destructive overlap while the quasi-monopolar negative waveform remains, as shown in Figure 24b,c. The high-amplitude quasi-monopolar negative pulses could be a powerful tool to investigate the effect of negative phases in cavitation-based applications, since cavitation is induced by the negative phase of ultrasound.

\subsubsection{Complex Ultrasound Wave Fronts by Binary Amplitude Hologram}

By controlling both the illuminating optical pulse shape and spatial illumination pattern to the photoacoustic absorber, researchers have achieved a very high degree of control over the resultant acoustic field both spatially and temporally, which is a significant challenge for conventional piezoelectric arrays. Brown et al. explored the availability of using binary amplitude 
(a)

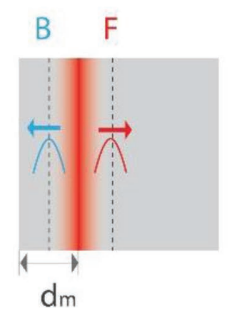

Forward (F)/backward (B) waves

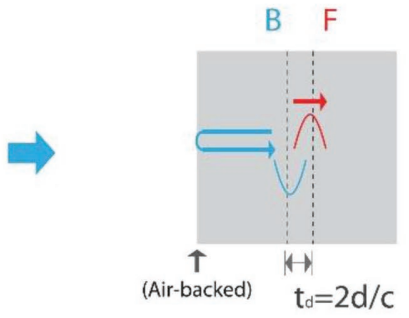

Reflected backward wave (B): polarity reversal ( $\pi$ phase shift)
B F

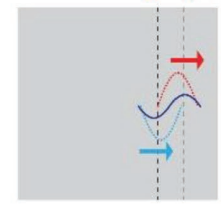

Phase cancellation (b)

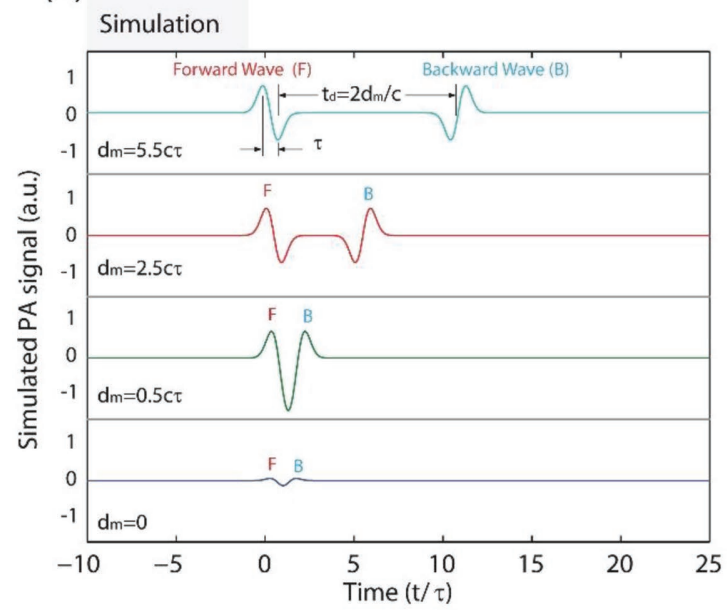

(c)

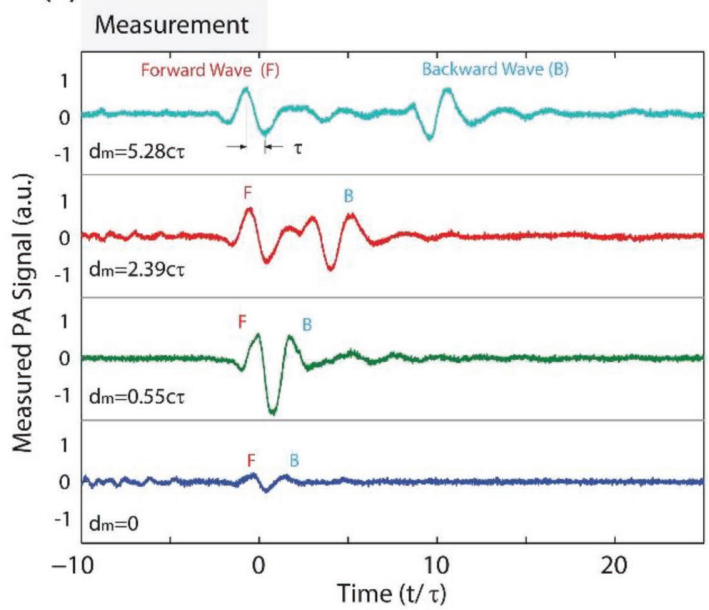

Figure 24. Quasi-monopolar photoacoustic (PA) pulses by photoacoustic phase cancellation. a) Schematic of the PA cancellation. Two oppositely propagating waves ( $F$, forward wave; $B$, backward wave). The reflected backward wave (B) with polarity reversal interferes with the original forwardgoing wave $(F)$. b) Calculated PA signals for different matching layer thicknesses $\left(d_{m}\right)$. c) Measured PA signals using PDMS-based absorbers. Adapted under a Creative Commons Attribution 4.0 International License. ${ }^{[64]}$ Copyright 2016, The Authors.

holograms (either on or off) to generate novel acoustic patterns including acoustic fields, as illustrated in Figure 25. ${ }^{[65]}$ These various acoustic fields can stimulate applications in particle manipulation, ultrasound therapy, reducing the acquisition time in ultrasound imaging and neural stimulation.

Two different algorithms were used to calculate the holograms: ray-based algorithm and direct binary search. The ray-based algorithm was implemented first to calculate the hologram for single focus, and the direct binary search was then used for calculating the holograms for more complicated target acoustic fields. For the former case, the initial acoustic field at the plane of hologram surface was inverse calculated from the focal point, resulting in a series of concentric circles-the Fresnel zone plate at the hologram plane. By setting the operating frequency, desired ring number, hologram aperture and focal length, the radius and distance between each ring can be defined. The researchers also explored the influence of the size of pixels on the hologram and of the repetition rate of the illuminating pulses on the focus pressure and size. It is not surprisingly to see finer meshed pixels on the hologram makes the focus size smaller and by matching the repetition rate of pulses to the travel time difference of acoustic wave from two neighboring rings can increase the focus pressure (to make waves from all rings focus at a same time). For the rest of the target acoustic fields, the researchers found the hologram by optimizing a cost function of the average pressure and standard variation of the target points/pattern from a totally randomized state. By pivoting all the pixels on the hologram and evaluating the cost function until no further improvement, they found the required hologram. They demonstrated these holograms by comparing to the simulation resultant acoustic field from the initial acoustic field same to the hologram state.

Experimentally, they fabricated such holograms with a laser cutter directly patterning on a transparent PMMA substrate. Afterward the surface was sprayed by black paint serving as the photoacoustic generation layer (Super Satin, Plasti Kote, Valspar). A PVDF needle hydrophone was scanned over the space to depict the acoustic field generated by single focus and line focused hologram showing an appropriate match with the simulation. However, it is worth-well to note that whatever these generated pattens are not only focused in one single plane, as shown in Figure 26. This is because the evaluation of the resultant field from the hologram was optimized for one single frequency and any harmonics will focus onto a similar pattern at different planes. Limited to the only freedom of the hologram (on and off state), no further optimization can be achieved to solve this problem. Furthermore, current holograms are not reprogrammable, and each pattern possesses gaps, that results 
(a)
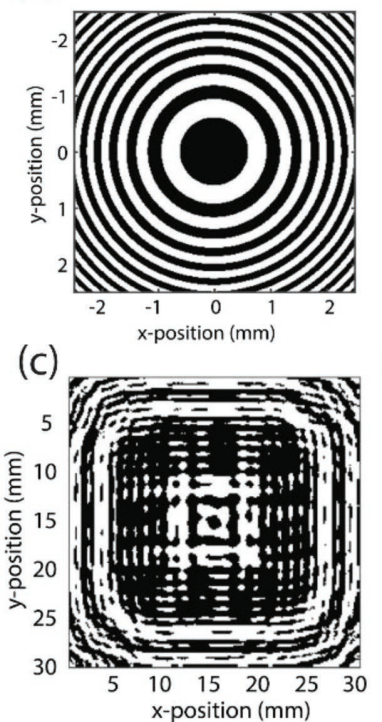

(b)

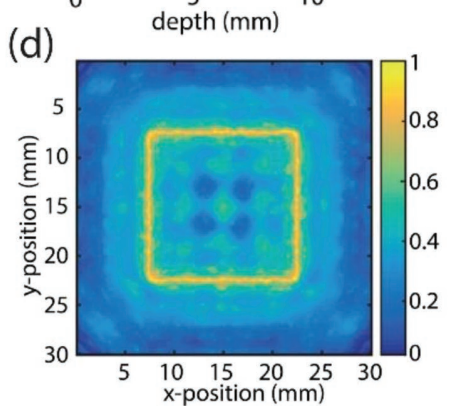

Figure 25. a) Single-focus Hologram that generated the largest focal width with the application of 40 pulses. b) Map of the maximum pressure field for a pulsing frequency of $5.7 \mathrm{MHz}$. c) Binary hologram designed to focus over the outline of a $1.5 \mathrm{~cm}$ square, in a plane $3 \mathrm{~cm}$ from the hologram. d) Maximum pressure in target plane of square generated by the hologram in c). Adapted under the terms of a Creative Commons Attribution (CC BY) license. ${ }^{[65]}$ Copyright 2016, The Authors.

in a portion of the incident optical radiation being transmitted into the medium. By implementing a special light modulator and illuminating the optical absorber with programmable patterns will make this hologram dynamic and more accurate.

(a)

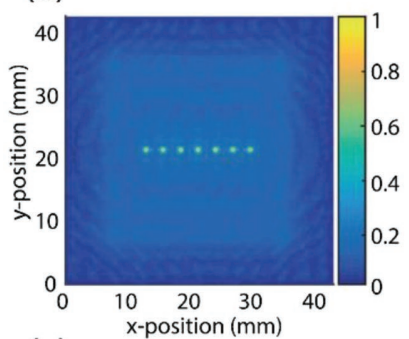

(c)

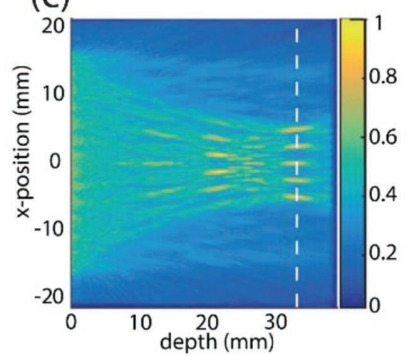

(b)

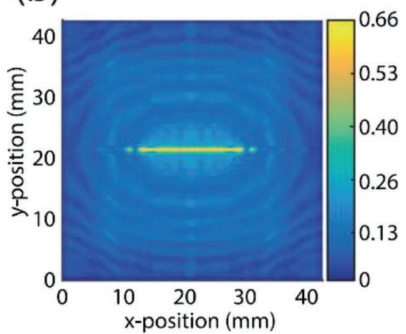

(d)

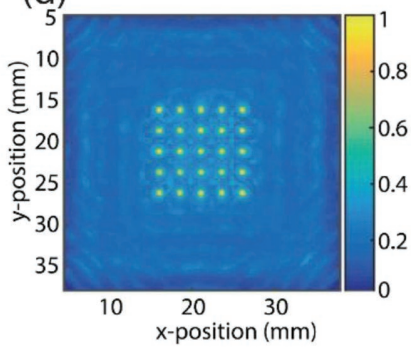

Figure 26. a) Maximum pressure generated using a point spacing of $5.55 \lambda$. b) Maximum pressure generated with a point spacing of $0.1675 \lambda$. c) Maximum pressure generated by hologram in $(x, z)$ plane. The dash line indicates target plane for array of foci. d) Maximum pressure for a $5 \times 5$ array of points. Adapted under the terms of a Creative Commons Attribution (CC BY) license. ${ }^{[65]}$ Copyright 2016, The Authors.

\subsubsection{Complex Ultrasound Wave Fronts by Tailored Optoacoustic Surface Profiles}

Brown et al. explored the availability of designing a 3D polymer surface profiles with random downhill binary search. ${ }^{[66]}$ Compared with the previous hologram design, a freedom of pixel height on the photoacoustic generation surface was added and the focal depth issue was alleviated. The algorithm is executed in short as below: First, the generation surface was restricted to a relative small thickness and pixelized, with each pixel of discretized height $h_{\mathrm{ij}}$, that was also discretized. Second, the inverse problem of finding the required source $p_{0}$ that created the target acoustic field was solved by optimizing the cost function of average pressure, standard deviation of temporal peak pressure, and a penalizing term. During the optimization, the $h_{\mathrm{ij}}$ was swept and pixels were randomly selected until cost function cannot be improved. To reduce the calculation complexity, an initial surface profile was set as a union of sets of spheres focused at the different points of the target acoustic pattern. Then the union profile was discretized. These sets of spheres, by an intuitive thought, should focus at different points and a combination of these points form the target pattern. Borrowing the 3D printing idea from W. Chan's work and they successfully created a focused pattern of "7" with transparent substrate of VeroClearRGD810 and optically absorbing polymer composite as the generation layer, as shown in Figure 27. The experimental measurement proved the simulation results from $\mathrm{k}$-Wave. It is worth to note that unlike previous hologram, the
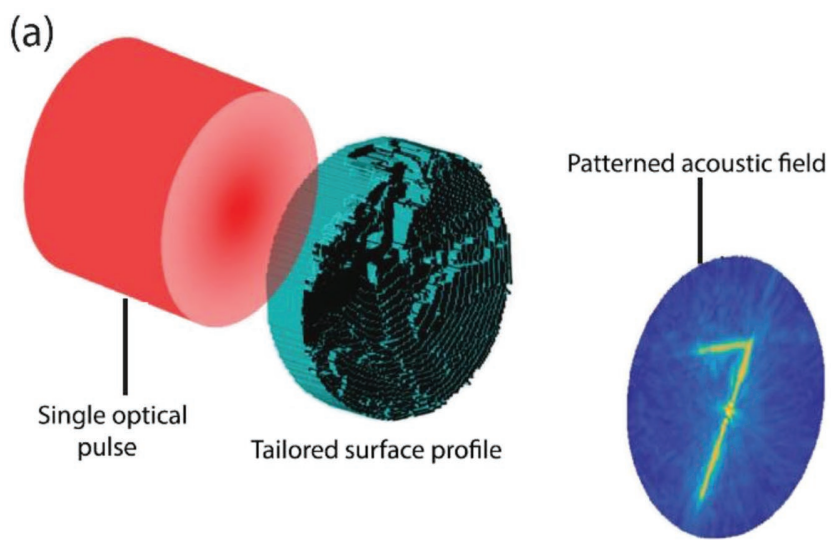

(b)

(c)

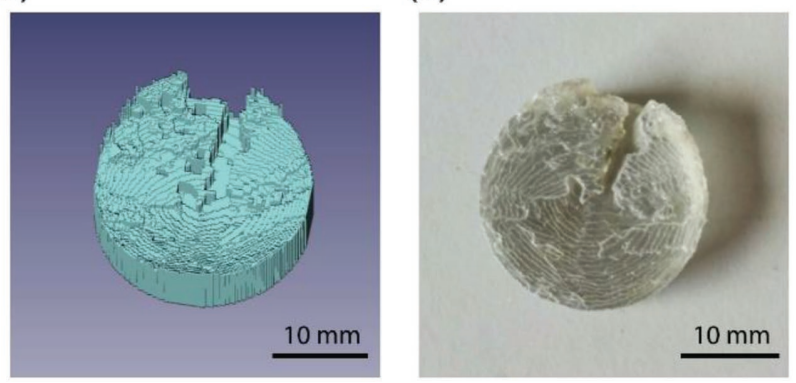

Figure 27. a) Surface profiles for patterned acoustic fields. b) Prepared surface mesh visualized using a software. c) Fabricated sample before absorber deposition. Adapted under the terms of a Creative Commons Attribution (CC BY) license. ${ }^{[66]}$ Copyright 2017, The Authors. 
pattern is focused on almost one plane. That may be due to the contribution of the new cost function and the initial phase added to each generation pixel.

\subsection{Challenges and Future Directions}

The nanocomposite structures have provided advanced technological platforms for efficient photoacoustic generation. These have shown great promises in realizing various applications. Despite such versatile uses, there are still challenges for improved performance and applications to in vivo and clinical environments. One challenge is to engineer photoacoustic materials with desired mechanical robustness such that one can increase pressure amplitudes by increasing input laser energy without any material damage. Also, despite the promise in nonthermal treatment using photoacoustic pulses, there are practical needs for thermal treatment using photoacoustic pulses. Another challenge arises because short photoacoustic pulses are significantly attenuated in tissue, preventing from moving toward clinical trials requiring treating tissue deep inside body.

\subsubsection{Mechanical Robustness Limiting Output Performance}

Mechanical robustness, quantitatively described by laser damage threshold, has been largely overlooked despite its great importance. ${ }^{[67]}$ The threshold for laser-induced ablation essentially determines an upper limit of input laser fluence onto transmitters and thus the maximum-available output pressure. Due to huge variation of the laser damage threshold for different types of transmitters, the maximum output pressure can change greatly up to 7-8 folds, which easily outweighs minute differences in optical absorption and energy conversion efficiency varying only on the order of a few tens of percentage. Improved nanocomposite characteristics can allow stronger output pressure, improve therapy effect, and elongate the treatment depth to target.

\subsubsection{Toward Thermal Treatment}

Photoacoustic generation from nanocomposite transmitters are suitable for nonthermal applications. For pulsed excitation, Nd:YAG laser beams are typically used with the following specification: 1-100 mJ pulse energy, 5-10 ns pulse width, and $<20 \mathrm{~Hz}$ repetition rate. Using these inputs, output pressure pulses are temporally sparse with a long pulse-to-pulse interval (e.g., $100 \mathrm{~ms}$ for $10 \mathrm{~Hz}$ repetition rate). With a focal configuration, LGFU has been utilized for nonthermal mechanical destruction of tissues (i.e., histotripsy), drug delivery, and cellular manipulation with microscale precision. With the input condition given by the above pulsed laser beam, pressure pulse trains of LGFU would produce an acoustic intensity of $<10 \mathrm{~mW} \mathrm{~cm}{ }^{-2}$ in spatial-peak temporal-average (SPTA). This can rarely deposit heat at a focal zone.

There are significant technological demands for thermal treatment such as hyperthermia or tissue coagulation. These are currently performed by high-intensity focused ultrasound using piezoelectric transducers in pulse burst or continuouswave modes. In order to make thermal deposition by LGFU, an input laser beam should be operated with $>1 \mathrm{~mJ}$ pulse $^{-1}$ and $>1 \mathrm{kHz}$ repetition rate to produce $>1 \mathrm{~W} \mathrm{~cm}^{-2}$ in SPTA. This requires higher specification than those available currently in commercial nanosecond pulsed laser systems. Even though pulsed laser performance is supported, there is an additional heating issue of nanocomposite transmitter because the $\mathrm{kHz}$ repetition laser pulses can accumulate thermal energy within the transmitter itself.

\subsubsection{Therapeutic Applications: Compensating Acoustic Attenuation in Tissue}

For therapeutic applications, nanocomposite transmitters have been used to produce LGFU with peak pressure of tens of MPa over a frequency range of $>15 \mathrm{MHz}$. Despite successful demonstration of microhistotripsy, drug release, cell detachment, and trans-membrane molecular delivery, all of these applications were realized using cultured cells and tissue samples prepared in vitro environments. Also, these commonly used water as an acoustic propagation medium with a low intrinsic attenuation coefficient $\left(2.2 \times 10^{-3} \mathrm{~dB} \mathrm{~cm}^{-1} \mathrm{MHz}^{-2}\right)$. Note that the intrinsic attenuation in tissues is more severe with an-order-of-magnitude higher coefficient, increasing with acoustic frequency and propagation depth. Due to the high-frequency operation of LGFU, the penetration depth would be limited significantly by such intrinsic attenuation in tissues (e.g., $<20 \mathrm{~mm}$ ). As LGFU-based therapy relies on formation of cavitation and its mechanical disruption at focus, tensile peak pressure amplitudes should be sufficiently high at a target depth, at least greater than the pressure threshold to generate cavitation (e.g., $-26 \mathrm{MPa}$ in water) after undergoing attenuation through tissue. This means that initial LGFU amplitudes from a curved transmitter should be further improved to perform cavitational therapy in vivo with a significant penetration depth.

\subsubsection{Low-Frequency Ultrasound Generation}

While broadband and high-frequency spectral output has been remarked as a major advantage of photoacoustic generation using nanocomposite transmitters, there are specific needs for reduced frequency characteristics. LGFU with a center frequency of $15 \mathrm{MHz}$ is proper for precision treatment of $<100 \mu \mathrm{m},{ }^{[67]}$ but it is clinically impractical to destroy a bulky target (e.g., kidney stone) greater than millimeter scale due to elongated operation time. An enlarged focal spot generated by a reduced operation frequency would be suitable to facilitate the treatment in time. Moreover, a low range of frequency $(\approx 0.5 \mathrm{MHz})$ has been often used for transcranial treatment using conventional piezoelectric focused ultrasound. However, it is challenging to fabricate nanocomposite transmitters with such low operation frequency. For lowering the frequency, photoacoustic pulse width can be broadened by longer laser pulse width, but this involves significant reduction in peak pressure as far-field photoacoustic waveforms are close to the time-derivative form of laser pulse. Although the same pulsed laser energy 
is given, peak pressure amplitudes can be greatly decreased as input laser pulses broaden. This means that an increased pulse laser energy should be provided to compensate the peak pressure reduction caused by the smooth laser pulse width.

\section{Conclusions and Outlook}

Efficient photoacoustic composites capable of generating highamplitude photoacoustic signals have allowed various interesting applications, which might be difficult to be realized with conventional piezoelectric-based approaches. Moreover, there has been a significant advance with development of photoacoustic transmitters to address the issues associated with their electrical counterparts. By employing high photoacoustic conversion efficiency and broad bandwidth, there will be more applications to be realized in near future. Particularly, miniature photoacoustic transmitters will be a game-changer in medical ultrasound, as fiber-optic photoacoustic imaging probes has already shown promise in guiding minimally invasive procedures. ${ }^{[51]}$ Another promising application will be ultrasound therapy by using a fiber-optic photoacoustic probes capable of delivering high-amplitude ultrasound. Fiber-optic photoacoustic transmitters in combination with a focusing scheme can generate sufficiently high-pressure amplitudes, which are desirable for some applications such as cavitation therapy as well as all-optical ultrasound imaging. Although photoacoustic transmitters were integrated with optical fibers, ${ }^{[10,11,30,51]}$ it is not easy to implement a focusing scheme due to fabrication challenges. There are only a few work reported for imaging ${ }^{[68]}$ and therapy. ${ }^{[67]}$ In addition, although there exists various efficient photoacoustic composites recently developed, one may need to further improve the conversion efficiencies of existing photoacoustic materials or find alternative photoacoustic materials with a higher conversion efficiency. The need may arise because the sizes of photoacoustic transmitters gets smaller for integration to a smaller aperture (e.g., fiber tip) and even with the same laser fluence the photoacoustic wave generated by the smaller aperture experiences the drastic magnitude decrease, compared to a larger aperture. Even with the help of a focusing scheme, a photoacoustic transmitter with a smaller aperture has a smaller focal gain. Another reason for the need is to take advantage of many compact laser systems recently developed. These compact lasers make photoacoustic transmitter systems very compact, but they typically deliver a high repetition rate of pulses, each pulse with a relatively low pulse energy.

\subsection{Outlook for Highly Efficient Photoacoustic Composites}

\subsubsection{High Photoacoustic Conversion}

From a light absorption perspective, light-absorbing fillers (e.g., carbon materials or metal nanoparticles) have enabled near perfect absorption for photoacoustic generation. Thus, photoacoustic generation in these materials is dominantly determined by the thermal expansion of materials. Since these fillers are embedded in polymers, exclusively PDMS for photoacoustic composite, the photoacoustic conversion efficiency of the composites is limited by the thermal expansion coefficient of PDMS. However, it is known that these PDMS-based composites have disadvantages, e.g., high temperature-induced performance degradation. The photoacoustic conversion efficiency of PDMS is decreased for high temperature. Evidently, the Grüneisen parameter of PDMS is reported to decrease with laser intensity. ${ }^{[69]}$ Therefore, to increase photoacoustic conversion efficiency, it is worth exploring other polymers having higher thermal expansion coefficient and material robustness. In addition, one can consider other photoacoustic generation mechanisms, i.e., nonlinear effects such as phase change and ablation, which are much more efficient than the thermoelastic effect. For example, liquid polymer-based composites for phase change could be effective when the liquid polymer is properly separated from water. Moreover, since heat transfer from light absorbers to the adjacent polymers plays an important role in efficient photoacoustic conversion, photoacoustic conversion efficiency can be enhanced by engineering the size and shape of light-absorbing fillers for decreasing a heat capacity ratio of the absorbers to the polymers (e.g., using nanoscale absorbers), or maximizing surface to volume ratio for enlarged heat dissipation pathways.

\subsubsection{Mechanical Robustness}

Photoacoustic amplitudes generated by optical excitation of photoacoustic materials increase with optical input power, which cannot be, however, too high because the material can be damaged. Thus, the material damages of photoacoustic composites limit allowable optical input power, thus dictating the maximum photoacoustic amplitudes. In this aspect, one can increase the maximum photoacoustic amplitude by improving the damage threshold of the composites. To do so, one may need to understand why such damages occur. There exist several potential causes to the material damages. One reason is that the materials damages are identified as delamination or ablation, which are believed to weak adhesion between the photoacoustic composites and the substrates. Another reason is that high thermal gradient induced by localized heating can cause the damages because of thermally induced strain difference within the polymers or between the composites and the substrate. Moreover, materials imperfections (e.g., small inhomogeneous regions) in the composites can cause to concentrate mechanical stress on the nearby regions, thereby initiating damages from the regions. One immediate solution is to increase the adhesion by increasing bonding forces through substrate treatments. Further systematic studies on the damage mechanism of photoacoustic composites are valuable.

\subsubsection{Fabrications}

Owing to significant progresses in nanofabrication technology, numerous light-absorbing fillers having good light absorption have be developed. These materials can be easily produced by many cost-effect methods. The key is how to fabricate a composite using these light-absorbing fillers. There 
have been many ways to form a composite; the methods include mixing, spin coating, and infiltration. For these conventional methods, to produce a thin composite layer with uniform dispersion of light-absorbing fillers, one may need polymer solution of low viscosity and surface functionalization of fillers. Apart from such easy-to-address requirements, the conventional approaches may not be applicable for certain applications using different types of substrates. Thus, other methods to produce a composite is necessary for active control of the composite thickness, which allows to tailor photoacoustic characteristics (e.g., bandwidths) depending on target applications.

\subsection{Outlook for Miniature Photoacoustic Transmitter}

Efficient photoacoustic materials are needed especially for applications requiring high-amplitude ultrasound, e.g., fiberbased photoacoustic probes for therapy. One major challenge is associated with the small acoustic element (typically, the diameter of optical fibers) of such transmitters. Photoacoustic signals leaving from the small element drastically decreases. To compensate this, fiber-based photoacoustic transmitters require higher input optical energy. However, too much optical energy delivered to the transmitters can cause the damages to the photoacoustic coating and/or the optical fibers. To avoid such an issue, one solution is to use a moderate optical energy and to find a photoacoustic material with a higher photoacoustic conversion efficiency for a desired signal amplitude. Also, to implement photoacoustic approaches for cavitation generation, it requires a high-pressure amplitude that exceeds cavitation threshold (e.g., negative pressure amplitude $>30 \mathrm{MPa}$ in water). Kim et al. reported ultrasound intravascular therapy by applying laser-generated-focused-ultrasound generated with a fiber-based photoacoustic transmitter. ${ }^{[67]}$ Instead of using a photoacoustic coating on a flat fiber tip, they use the fiber head attached by a fabricated concave structure with a photoacoustic composite coating, thus taking advantage of the focal gain of the concave surface and generating a strong pressure amplitude at the focus. They were able to generate a positive peak of $10.9 \mathrm{MPa}$ and negative peak of $5.7 \mathrm{MPa}$ at the laser fluence of $1.5 \mathrm{~mJ} \mathrm{~cm}{ }^{-2}$. Even though the negative peak was smaller than the inertial cavitation threshold, they showed that the positive peak was edlarge enough to create localized mechanical effects (e.g., rupture of exogeneous microbubbles). They found that compared with a flat tip fiber with a carbon black/PDMS coating, the pressure amplitude generated by the focused transmitter was 15 times higher due to both the gain of the focusing as well as the larger conversion efficiency of a CS/PDMS composite. This approach using the focused fiber-optic transmitter will be more attractive for therapy when it can generate inertial cavitation without the needs of the injected bubble nuclei. However, to exceed cavitation threshold (>25 MPa) in water, at least fivefold larger inci-

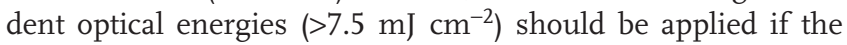
same system is used. It remains an open question whether the photoacoustic coating can be improved to ensure such high laser fluences. In this aspect, further studies on the damage threshold of photoacoustic composites and photoacoustic conversion are needed.

\section{Acknowledgements}

T.L. and H.W.B. contributed equally to this work. The authors thank MCube program, NSF (1825502), and NIH for the support of this work. H.W.B. acknowledges the support from the Technology Innovation Program (No. 10052749) funded by the Ministry of Trade, Industry, and Energy of Korea. This article is part of the Advanced Optical Materials Hall of Fame article series, which recognizes the excellent contributions of leading researchers to the field of optical materials science.

\section{Conflict of Interest}

The authors declare no conflict of interest.

\section{Keywords}

photoacoustic composites, photoacoustic transmitters, laser-generated ultrasound

Received: April 15, 2018

Revised: July 15, 2018

Published online: August 23, 2018

[1] D. M. McCann, M. C. Forde, NDT\&E Int. 2001, 34, 71.

[2] L. V. Wang, Photoacoustic Imaging and Spectroscopy, CRC Press, Boca Raton, FL 2009.

[3] A. de la Zerda, J.-W. Kim, E. I. Galanzha, S. S. Gambhir, V. P. Zharov, Contrast Media Mol. Imaging 2011, 6, 346.

[4] Q. Zhang, N. Iwakuma, P. Sharma, B. M. Moudgil, C. Wu, J. McNeill, H. Jiang, S. R. Grobmyer, Nanotechnology 2009, 20, 395102.

[5] A. Agarwal, S. W. Huang, M. O'Donnell, J. Appl. Phys. 2007, 102, 064701

[6] H. Yang, J.-S. Kim, S. Ashkenazi, M. O’Donnell, L. J. Guo, Appl. Phys. Lett. 2006, 89, 093901.

[7] H. W. Baac, J. G. Ok, H. J. Park, T. Ling, S.-L. Chen, A. J. Hart, L. J. Guo, Appl. Phys. Lett. 2010, 97, 234104.

[8] W. Y. Chang, W. B. Huang, J. Kim, S. B. Li, X. N. Jiang, Appl. Phys. Lett. 2015, 107, 161903.

[9] B.-Y. Hsieh, J. Kim, J. Zhu, S. Li, X. Zhang, X. Jiang, Appl. Phys. Lett. 2015, 106, 021902.

[10] R. J. Colchester, C. A. Mosse, D. S. Bhachu, J. C. Bear, C. J. Carmalt, I. P. Parkin, B. E. Treeby, I. Papakonstantinou, A. E. Desjardins, Appl. Phys. Lett. 2014, 104, 173502.

[11] S. Noimark, R. J. Colchester, B. J. Blackburn, E. Z. Zhang, E. J. Alles, S. Ourselin, P. C. Beard, I. Papakonstantinou, I. P. Parkin, A. E. Desjardins, Adv. Funct. Mater. 2016, 26, 8390.

[12] H. W. Baac, J. G. Ok, A. Maxwell, K.-T. Lee, Y.-C. Chen, A. J. Hart, Z. Xu, E. Yoon, L. J. Guo, Sci. Rep. 2012, 2, 989.

[13] Y. Hou, S. Ashkenazi, S. W. Huang, M. O'Donnell, IEEE Trans. Ultrason. Ferroelectr. Freq. Control 2008, 55, 2719.

[14] T. Buma, M. Spisar, M. O'Donnell, Appl. Phys. Lett. 2001, 79, 548.

[15] S. H. Lee, M.-a. Park, J. J. Yoh, H. Song, E. Y. Jang, Y. H. Kim, S. Kang, Y. S. Yoon, Appl. Phys. Lett. 2012, 101, 241909.

[16] D. Kim, M. Ye, C. P. Grigoropoulos, Appl. Phys. A: Mater. Sci. Process. 1998, 67, 169.

[17] G. J. Diebold, T. Sun, M. I. Khan, Phys. Rev. Lett. 1991, 67, 3384.

[18] M. H. Xu, L. H. V. Wang, Rev. Sci. Instrum. 2006, 77, 041101.

[19] V. E. Gusev, A. A. Karabutov, Laser Optoacoustics, American Institute of Physics, New York 1993

[20] T. Sun, G. J. Diebold, Nature 1992, 355, 806.

[21] I. G. Calasso, W. Craig, G. J. Diebold, Phys. Rev. Lett. 2001, 86, 3550. 
[22] M. I. Khan, T. Sun, G. J. Diebold, J. Acoust. Soc. Am. 1993, 93, 1417.

[23] J. E. Alba-Rosales, G. Ramos-Ortiz, L. F. Escamilla-Herrera, B. Reyes-Ramirez, L. Polo-Parada, G. Gutierrez-Juarez, Appl. Phys. Lett. 2018, 112, 143101.

[24] A. Prost, F. Poisson, E. Bossy, Phys. Rev. B 2015, 92, 115450.

[25] Y. J. Shi, H. Qin, S. H. Yang, D. Xing, Nano Res. 2016, 9, 3644.

[26] B. T. Cox, P. C. Beard, J. Acoust. Soc. Am. 2005, 117, 3616.

[27] F. Gao, R. Kishor, X. Feng, S. Liu, R. Ding, R. Zhang, Y. Zheng, Photoacoustics 2017, 7, 1.

[28] H. F. Shi, J. G. Ok, H. W. Baac, L. J. Guo, Appl. Phys. Lett. 2011, 99, 211103.

[29] H. W. Baac, J. G. Ok, T. Lee, L. J. Guo, Nanoscale 2015, 7, 14460.

[30] R. K. Poduval, S. Noimark, R. J. Colchester, T. J. Macdonald, I. P. Parkin, A. E. Desjardins, I. Papakonstantinou, Appl. Phys. Lett. 2017, 110, 223701.

[31] Z. Chen, Y. Wu, Y. Yang, J. Li, B. Xie, X. Li, S. Lei, J. Ou-Yang, X. Yang, Q. Zhou, B. Zhu, Nano Energy 2018, 46, 8

[32] E. Biagi, F. Margheri, D. Menichelli, IEEE Trans. Ultrason. Ferroelectr. Freq. Control 2001, 48, 1669.

[33] L. Yuan, J. Dai, X. Fan, T. Song, Y. T. Tao, K. Wang, Z. Xu, J. Zhang, X. Bai, P. Lu, J. Chen, J. Zhou, Z. L. Wang, ACS Nano 2011, 5, 4007.

[34] J. Heremans, I. Rahim, M. S. Dresselhaus, Phys. Rev. B 1985, 32, 6742.

[35] Y. Hou, J. S. Kim, S. Ashkenazi, S. W. Huang, L. J. Guo, M. O'Donnell, Appl. Phys. Lett. 2007, 91, 073507.

[36] N. Wu, Y. Tian, X. T. Zou, V. Silva, A. Chery, X. W. Wang, J. Opt. Soc. Am. B 2012, 29, 2016.

[37] S. G. Park, S. B. Yang, M. S. Ahn, Y. J. Oh, Y. T. Kim, K. H. Jeong, Nanoscale 2016, 8, 757

[38] S. Kang, Y. Yoon, J. Kim, W. Kim, Appl. Phys. Lett. 2013, 102, 021908.

[39] T. Lee, L. J. Guo, Adv. Opt. Mater. 2017, 5, 1600421.

[40] G. Yoo, Y. Park, P. Sang, H. W. Baac, J. Heo, Opt. Mater. Express 2016, 6, 2567.

[41] Q. S. Li, X. M. Zhao, S. L. Fang, F. N. Liang, Z. Chen, in 16th International Conference on Numerical Simulation of Optoelectronic Devices (NUSOD), University of Sydney, Australia 2016, pp. 169-170.

[42] T. Lee, J. G. Ok, L. J. Guo, H. W. Baac, Appl. Phys. Lett. 2016, 108, 104102.

[43] J. Di, J. Kim, Q. Y. Hu, X. N. Jiang, Z. Gu, J. Controlled Release 2015, 220, 592.

[44] T. Lee, H. W. Baac, J. G. Ok, H. S. Youn, L. J. Guo, Phys. Rev. Appl. 2014, 2, 024007.

[45] H. W. Baac, J. Frampton, J. G. Ok, S. Takayama, L. J. Guo, J. Biophotonics 2013, 18, 6 .

[46] H. W. Baac, T. Lee, L. J. Guo, Biomed. Opt. Express 2013, 4, 1442.

[47] T. Lee, W. Luo, Q. C. Li, H. Demirci, L. J. Guo, Small 2017, 13, 1701555.
[48] T. Lee, H. W. Baac, J. G. Ok, H. S. Youn, L. J. Guo, Phys. Rev. Appl. 2015, 3, 044007

[49] E. J. Alles, R. J. Colchester, A. E. Desjardins, IEEE Trans. Ultrason. Ferroelectr. Freq. Control 2016, 63, 83.

[50] Y.-C. Chen, H. W. Baac, K.-T. Lee, S. Fouladdel, K. Teichert, J. G. Ok, Y.-H. Cheng, P. N. Ingram, A. J. Hart, E. Azizi, L. J. Guo, M. S. Wicha, E. Yoon, ACS Nano 2017, 11, 4660.

[51] R. J. Colchester, E. Z. Zhang, C. A. Mosse, P. C. Beard, I. Papakonstantinou, A. E. Desjardins, Biomed. Opt. Express 2015, 6, 1502.

[52] M. C. Finlay, C. A. Mosse, R. J. Colchester, S. Noimark, E. Z. Zhang, S. Ourselin, P. C. Beard, R. J. Schilling, I. P. Parkin, I. Papakonstantinou, A. E. Desjardins, Light: Sci. Appl. 2017, 6, 17103.

[53] W. Xia, S. Noimark, S. Ourselin, S. J. West, M. C. Finlay, A. L. David, A. E. Desjardins, in Medical Image Computing and Computer-Assisted Intervention - MICCAI 2017 (Eds: M. Descoteaux, L. Maier-Hein, A. Franz, P. Jannin, D. L. Collins, S. Duchesne), Springer International Publishing, Switzerland 2017, p. 10434.

[54] S. L. Chen, Appl. Sci. 2017, 7, 25

[55] S. Noimark, R. J. Colchester, R. K. Poduval, E. Maneas, E. J. Alles, T. Zhao, E. Z. Zhang, M. Ashworth, E. Tsolaki, A. H. Chester, N. Latif, S. Bertazzo, A. L. David, S. Ourselin, P. C. Beard, I. P. Parkin, I. Papakonstantinou, A. E. Desjardins, Adv. Funct. Mater. 2018, 28, 1704919.

[56] E. Zhang, J. Laufer, P. Beard, Appl. Opt. 2008, 47, 561.

[57] T. Ling, S. L. Chen, L. J. Guo, Appl. Phys. Lett. 2011, 98, 204103.

[58] C. Zhang, T. Ling, S. L. Chen, L. J. Guo, ACS Photonics 2014, 1, 1093.

[59] H. Li, B. Q. Dong, Z. Zhang, H. F. Zhang, C. Sun, Sci. Rep. 2014, 4, 4496.

[60] S.-L. Chen, Y.-C. Chang, C. Zhang, J. G. Ok, T. Ling, M. T. Mihnev, T. B. Norris, L. J. Guo, Nat. Photonics 2014, 8, 537.

[61] J. A. Guggenheim, J. Li, T. J. Allen, R. J. Colchester, S. Noimark, O. Ogunlade, I. P. Parkin, I. Papakonstantinou, A. E. Desjardins, E. Z. Zhang, P. C. Beard, Nat. Photonics 2017, 11, 714.

[62] M. Mohammadzadeh, S. R. Gonzalez-Avila, Y. C. Wan, X. Wang, H. Zheng, C. D. Ohl, Appl. Phys. Lett. 2016, 108, 024101.

[63] W. W. Chan, T. Hies, C. D. Ohl, Appl. Phys. Lett. 2016, 109, 174102.

[64] T. Lee, Q. Li, L. J. Guo, Sci. Rep. 2016, 6, 21511.

[65] M. D. Brown, J. Jaros, B. T. Cox, B. E. Treeby, J. Acoust. Soc. Am 2016, 139, 1637

[66] M. D. Brown, D. I. Nikitichev, B. E. Treeby, B. T. Cox, Appl. Phys. Lett. 2017, 110, 094102

[67] J. Kim, W. Y. Chang, H. Y. Wu, X. N. Jiang, IEEE Int. Ultrason. Symp. Washington DC, WA, USA, September 2017

[68] E. J. Alles, S. Noimark, E. Zhang, P. C. Beard, A. E. Desjardins, Biomed. Opt. Express 2016, 7, 3696.

[69] E. Petrova, S. Ermilov, R. Su, V. Nadvoretskiy, A. Conjusteau, A. Oraevsky, Opt. Express 2013, 21, 25077. 